\title{
Electrochemical-induced Transfer Hydrogenation of Imidazopyridines with Secondary Amine as Hydrogen Donor
}

Jiangwei Wen,,* Hongyun Qin, Kelu Yan, Xiaoting Yang, Xuejun Sun, Wei Wei, Jianjing Yang* Hua Wang*

Institute of Medicine and Materials Applied Technologies, College of Chemistry and Chemical Engineering, Qufu Normal University, Qufu, Shandong 273165, P. R. China.

E-mail: wenjy@,qfnu.edu.cn, jiyang@,whu.edu.cn, huawang@qfnu.edu.cn 


\section{Contents}

$\begin{array}{ll}\text { 1. General information } & \text { S3 }\end{array}$

2. General procedure for electrochemical-induced transfer hydrogenation of imidazopyridines with secondary amine as hydrogen donor

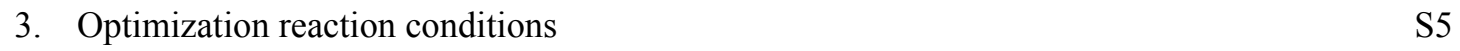

$\begin{array}{ll}\text { 4. Gram-scale experiments } & \text { S6 }\end{array}$

$\begin{array}{ll}\text { 5. Preliminary mechanistic studies } & \text { S6 }\end{array}$

(1) Deuteration experiments $\quad$ S6

(2) Radical trapping experiments $\quad$ S8

$\begin{array}{ll}\text { (3) CV experiments } & \text { S9 }\end{array}$

6. References $\quad \mathrm{S} 10$

$\begin{array}{ll}\text { 7. Detail descriptions for products } & \mathrm{S} 10\end{array}$

$\begin{array}{ll}\text { 8. Copies of product NMR and HRMS Spectra } & \text { S17 }\end{array}$ 


\section{General information}

All glassware was oven dried at $100{ }^{\circ} \mathrm{C}$ for hours and cooled down under vacuum. Imidazopyridines was prepared according to reported procedures. ${ }^{1}$ All the reaction prepared using the solvent of $\mathrm{CH}_{3} \mathrm{CN}$ (99.9\%, Extra Dry with molecular sieves, Water $\left.\leq 50 \mathrm{ppm}\right)$ was purchased from Innochem. The deoxygenation of dideionized water and $\mathrm{CH}_{3} \mathrm{CN}$ is through Schlenk technology. Unless otherwise noted, materials were obtained from commercial suppliers and used without further purification. The instrument for electrolysis is dual display potentiostat (DJS-292B) (made in China), the carbon rod (d: $6 \mathrm{~mm}), \operatorname{Pt}\left(1 \times 1 \mathrm{~cm}^{2}\right)$, and $\mathrm{Ni}\left(1 \times 1 \mathrm{~cm}^{2}\right)$ was purchased from Xuzhou Xinke Instrument and Meter Co. LTD. The thin layer chromatography (TLC) employed glass $0.25 \mathrm{~mm}$ silica gel plates. Flash chromatography columns were packed with 200-300 mesh silica gel in petroleum (b. p. $60-90{ }^{\circ} \mathrm{C}$ ). ${ }^{1} \mathrm{H}$ and ${ }^{13} \mathrm{C}$ NMR data were recorded with Bruker Advance III (500 MHz) spectrometers with tetramethylsilane as an internal standard. All chemical shifts $(\delta)$ are reported in ppm and coupling constants $(J)$ in Hz. All chemical shifts are reported relative to tetramethylsilane and d-solvent peaks (77.00 ppm, chloroform), respectively. 
2. General procedure for electrochemical-induced transfer hydrogenation of imidazopyridines with secondary amine as hydrogen donor.

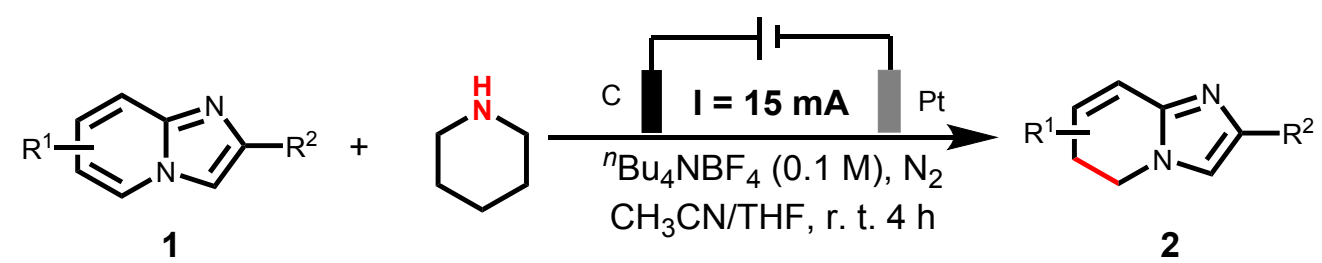

In an oven-dried undivided three-necked bottle $(25 \mathrm{~mL})$ equipped with a stir bar, imidazopyridine $1(0.25 \mathrm{mmol})$ and ${ }^{\mathrm{n}} \mathrm{Bu}_{4} \mathrm{NBF}_{4}(1.0 \mathrm{mmol}, 330 \mathrm{mg})$ were combined and added. The bottle was equipped with graphite rod $(\mathrm{d}: 6.0 \mathrm{~mm})$ anode and platinum plate $\left(1 \times 1 \mathrm{~cm}^{2}\right)$ cathode (distance between electrodes $(5-10 \mathrm{~mm})$ ) and was then charged with nitrogen. Under the protection by nitrogen, piperdine $(0.75 \mathrm{mmol})$, THF $(1.0 \mathrm{~mL})$ and $\mathrm{CH}_{3} \mathrm{CN}(9.0 \mathrm{~mL})$ were slowly injected into the reaction tube. The reaction mixture was stirred and electrolyzed at a constant current of $15 \mathrm{~mA} \mathrm{~cm}^{-2}$ under room temperature for $4 \mathrm{~h}$. When the reaction was finished, the reaction mixture was washed with water and extracted with $\mathrm{CH}_{2} \mathrm{Cl}_{2}(10 \mathrm{~mL} \times 3)$. The organic layers were combined, dried over $\mathrm{Na}_{2} \mathrm{SO}_{4}$, and concentrated. The pure product was obtained by flash column chromatography on silica gel (petroleum: ethyl ether $=3: 1$ $10: 1)$

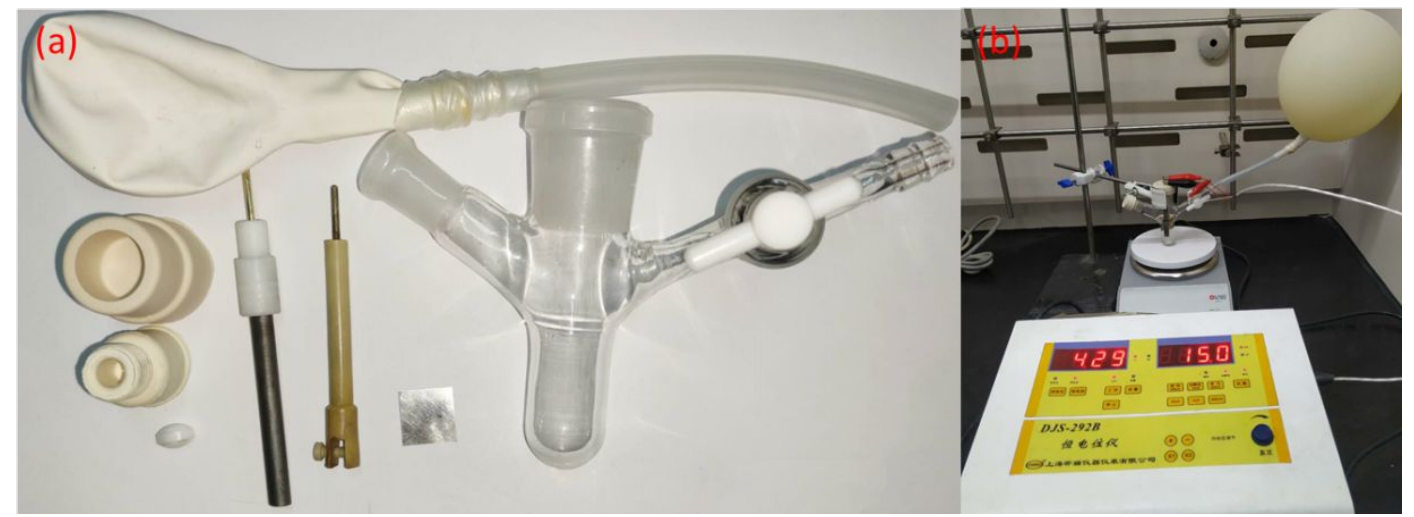

Fig. S1. Set-up of Experiments (the photographs come from our laboratory). (a) Experimental equipment. (b) Reaction process diagram 


\section{Optimization reaction conditions}

Table S1. Investigation of solvent effects ${ }^{a}$

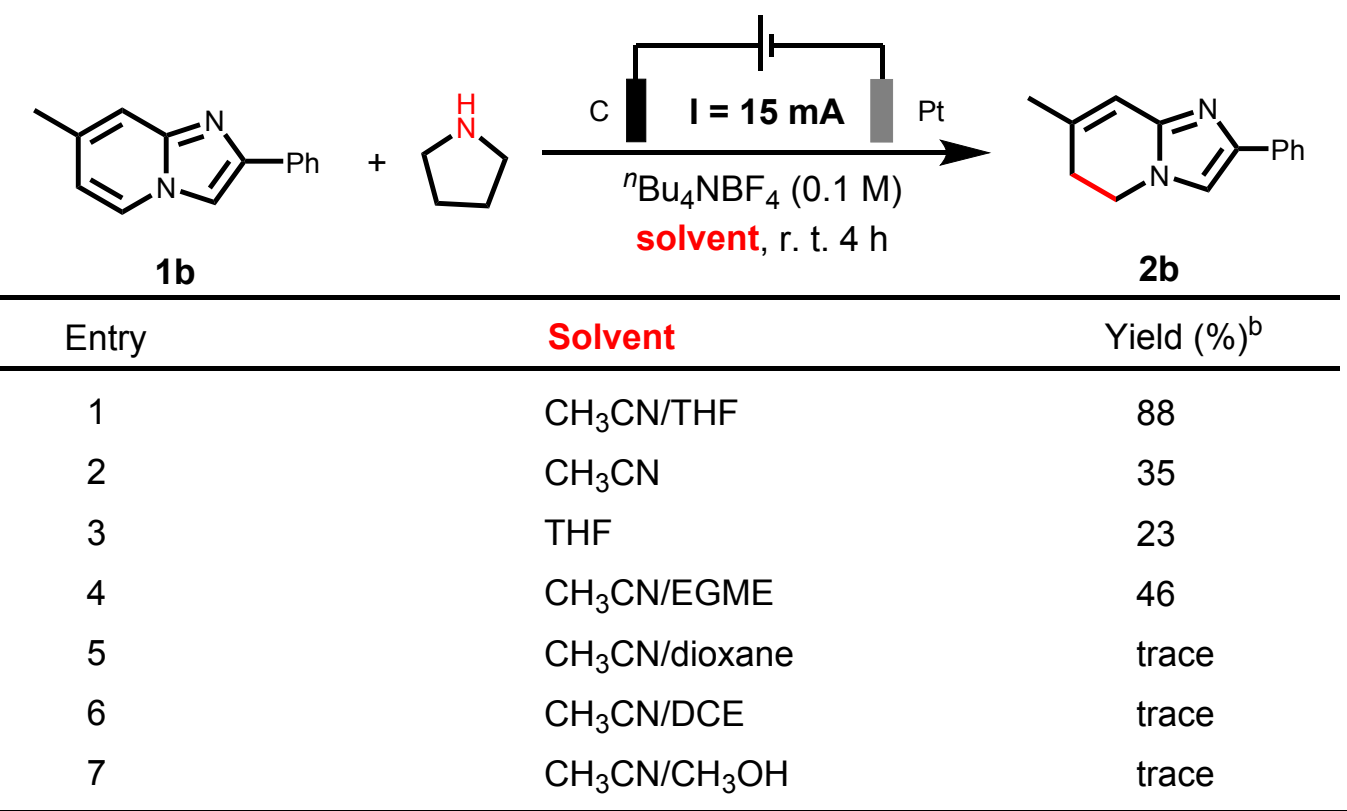

${ }^{a}$ Standard conditions: $\mathrm{C}$ anode, $\mathrm{Pt}$ cathode, constant current $=15 \mathrm{~mA}, \mathbf{1 b}(0.25 \mathrm{mmol}, 52.0 \mathrm{mg})$, piperdine $(0.75 \mathrm{mmol}, 69.0 \mu \mathrm{l}),{ }^{n} \mathrm{BuNBF}_{4}(4.0$ equiv, $330.0 \mathrm{mg})$, Solvent $(10.0 \mathrm{~mL}, \mathrm{~V}=9: 1)$, r. t., $\mathrm{N}_{2}, 4$ h. EGME = ethylene glycol monomethylether, DCE $=1,2$-dichloroethane. ${ }^{b}$ Isolated yields.

Scheme S1. Investigation of different secondary amines

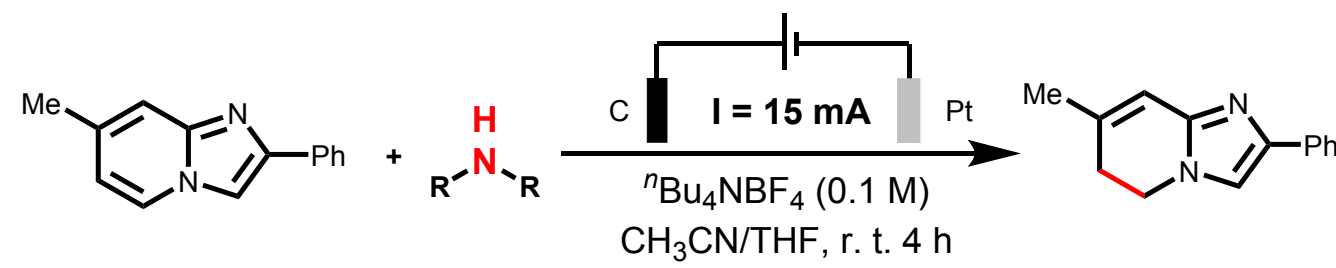

$1 \mathrm{~b}$

$2 b$<smiles>C1CCNC1</smiles>

$88 \%$<smiles>c1cc[nH]c1</smiles>

trace<smiles>C1CCNCC1</smiles>

$92 \%$<smiles>CC1(C)CCCC(C)(C)N1</smiles>

$20 \%$<smiles>c1ccc2c(c1)CCNC2</smiles>

$15 \%$

$\begin{array}{cc}\mathrm{NH}_{3} \cdot \mathrm{H}_{2} \mathrm{O} & \stackrel{\mathrm{N}}{\text { in water }} \\ 27 \% & 30 \%\end{array}$<smiles>CNCCNC</smiles><smiles>c1ccc(Nc2ccccc2)cc1</smiles>

$\mathrm{Et}_{3} \mathrm{~N}$ $22 \%$

n. d.

${ }^{a}$ Standard conditions: $\mathrm{C}$ anode, Pt cathode, constant current $=15 \mathrm{~mA}, \mathbf{1 b}(0.25 \mathrm{mmol}, 52.0$ $\mathrm{mg}$ ), amines $(0.75 \mathrm{mmol}, 69.0 \mu \mathrm{l}),{ }^{n} \mathrm{BuNBF}_{4}(4.0$ equiv, $330.0 \mathrm{mg})$, Solvent $(10.0 \mathrm{~mL}, \mathrm{~V}=9$ : 1), r. t., $\mathrm{N}_{2}, 4$ h. ${ }^{b}$ Isolated yields. 


\section{Gram-scale experiments}

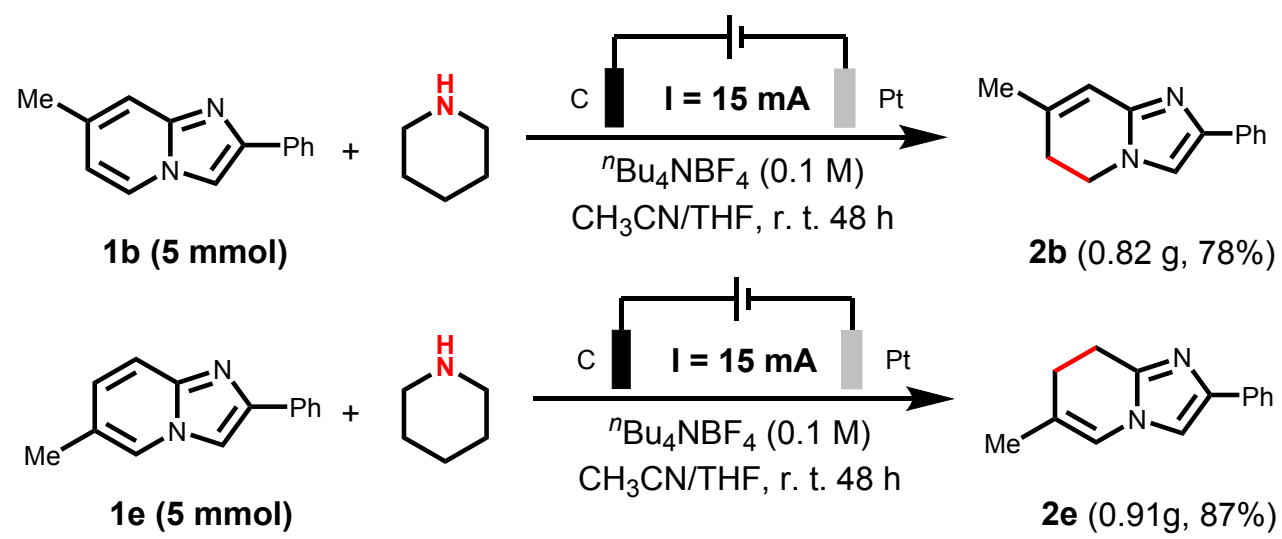

In an oven-dried undivided three-necked bottle $(150 \mathrm{~mL})$ equipped with a stir bar, $\mathbf{1 b}(5 \mathrm{mmol}$, $1.04 \mathrm{~g})$ or $1 \mathrm{e}(5 \mathrm{mmol}, 1.04 \mathrm{~g})$ and ${ }^{\mathrm{n}} \mathrm{Bu}_{4} \mathrm{NBF}_{4}(10.0 \mathrm{mmol}, 3.3 \mathrm{~g})$ were combined and added. The bottle was equipped with graphite rod $(\mathrm{d}: 6.0 \mathrm{~mm})$ anode and platinum plate $\left(1 \times 1 \mathrm{~cm}^{2}\right)$ cathode and was then charged with nitrogen. Under the protection by nitrogen, piperdine (15.0 mmol, 1.38 $\mathrm{mL}), \mathrm{THF} / \mathrm{CH}_{3} \mathrm{CN}(100 \mathrm{~mL}, 1: 9)$ were slowly injected into the reaction tube. The reaction mixture was stirred and electrolyzed at a constant current of $15 \mathrm{~mA} \mathrm{~cm}^{-2}$ under room temperature for $48 \mathrm{~h}$. When the reaction was finished, the reaction mixture was washed with water and extracted with $\mathrm{CH}_{2} \mathrm{Cl}_{2}(10 \mathrm{~mL} \times 3)$. The organic layers were combined, dried over $\mathrm{Na}_{2} \mathrm{SO}_{4}$, and concentrated. The pure product was obtained in $78 \%(\mathbf{2 b}, 0.82 \mathrm{~g})$ and $87 \%(\mathbf{2 e}, 0.91 \mathrm{~g})$ yield by flash column chromatography on silica gel (petroleum: ethyl ether $=7: 1$ ).

\section{Preliminary mechanistic studies}

(1) Deuteration experiments

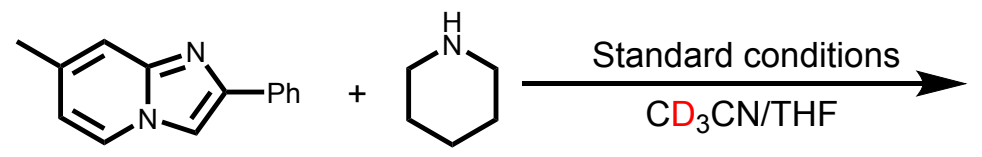

$1 \mathrm{~b}$<smiles>CC1=Cc2nc(-c3ccccc3)cn2C(O)(c2ccccc2)C1O</smiles>

yield: $75 \%$ $\mathrm{D}=0 \%$

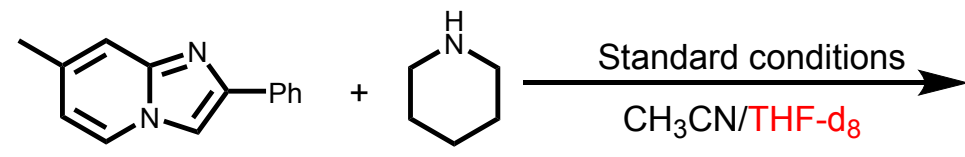

$1 b$<smiles>CC1=Cc2nc(-c3ccccc3)cn2C(O)(c2ccccc2)C1O</smiles>

yield: $84 \%$ $\mathrm{D}=0 \%$

In an oven-dried undivided three-necked bottle $(25 \mathrm{~mL})$ equipped with a stir bar, 7-methyl-2-phenyl-5,6-dihydroimidazo[1,2-a]pyridine $\mathbf{1 b}(0.25 \mathrm{mmol}, 52.0 \mathrm{mg})$ and ${ }^{\mathrm{n}} \mathrm{Bu}_{4} \mathrm{NBF}_{4}$ 
$(1.0 \mathrm{mmol}, 330.0 \mathrm{mg})$ were combined and added. The bottle was equipped with graphite rod (d: $6.0 \mathrm{~mm})$ anode and platinum plate $\left(1 \times 1 \mathrm{~cm}^{2}\right)$ cathode and was then charged with nitrogen. Under the protection by nitrogen, piperdine $(0.75 \mathrm{mmol}, 69.0 \mu \mathrm{l}), \mathrm{THF} / \mathrm{CD}_{3} \mathrm{CN}(10 \mathrm{~mL}, 1: 9)$ or THF- $\mathrm{d}_{8} / \mathrm{CH}_{3} \mathrm{CN}$ ((10 mL, 1:9) were slowly injected into the reaction tube. The reaction mixture was stirred and electrolyzed at a constant current of $15 \mathrm{~mA} \mathrm{~cm}^{-2}$ under room temperature for $4 \mathrm{~h}$. When the reaction was finished, the reaction mixture was washed with water and extracted with $\mathrm{CH}_{2} \mathrm{Cl}_{2}(10 \mathrm{~mL} \times 3)$. The organic layers were combined, dried over $\mathrm{Na}_{2} \mathrm{SO}_{4}$, and concentrated. The pure product was obtained in $75 \%$ and $84 \%$ yield by flash column chromatography on silica gel (petroleum: ethyl ether $=3: 1$ ). The ${ }^{1} \mathrm{HNMR}$ results show that $\mathrm{D}$ substitution were $0 \%$.

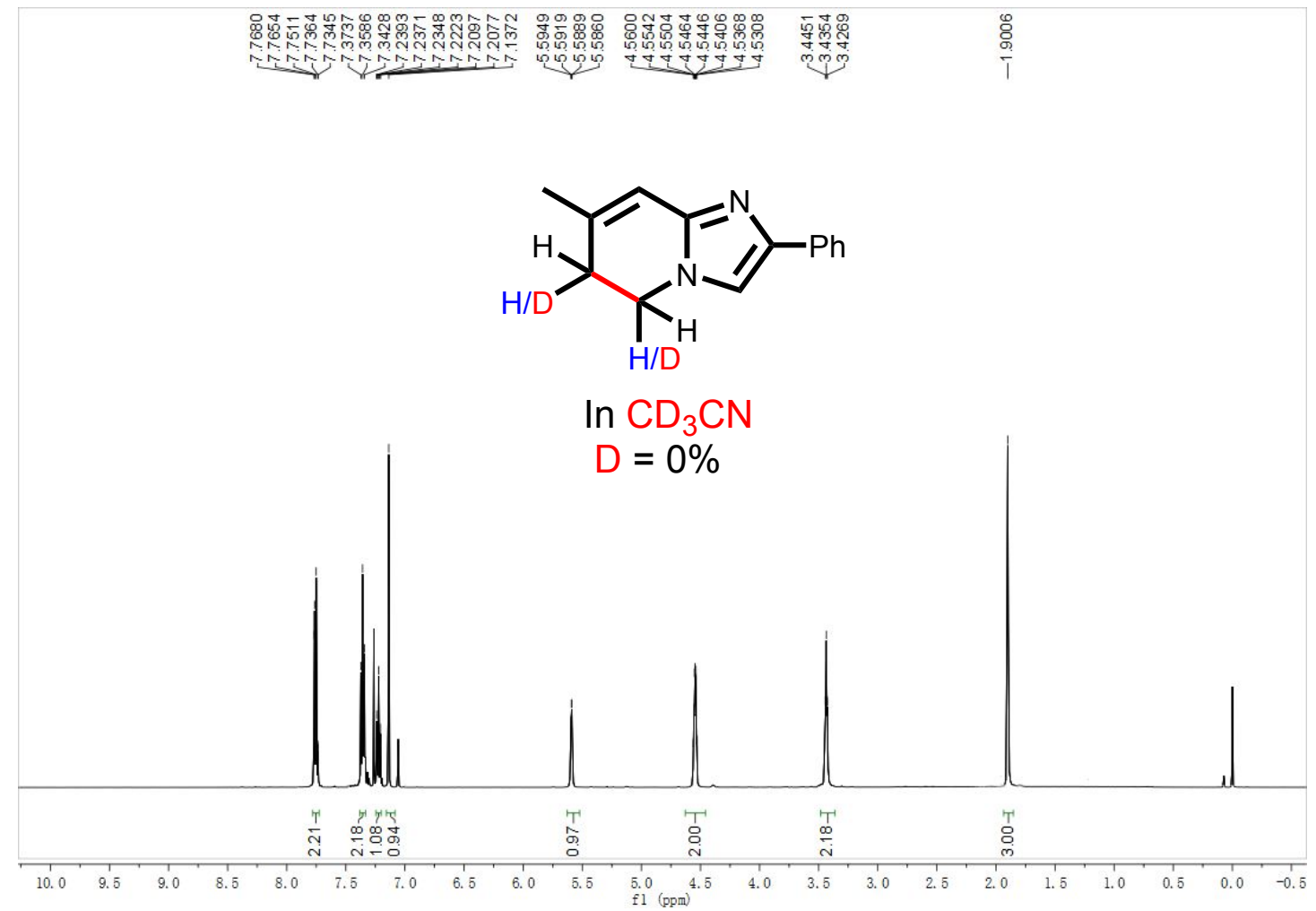

Fig. S2. Deuteration experiments results of $\mathrm{CD}_{3} \mathrm{CN}$. 


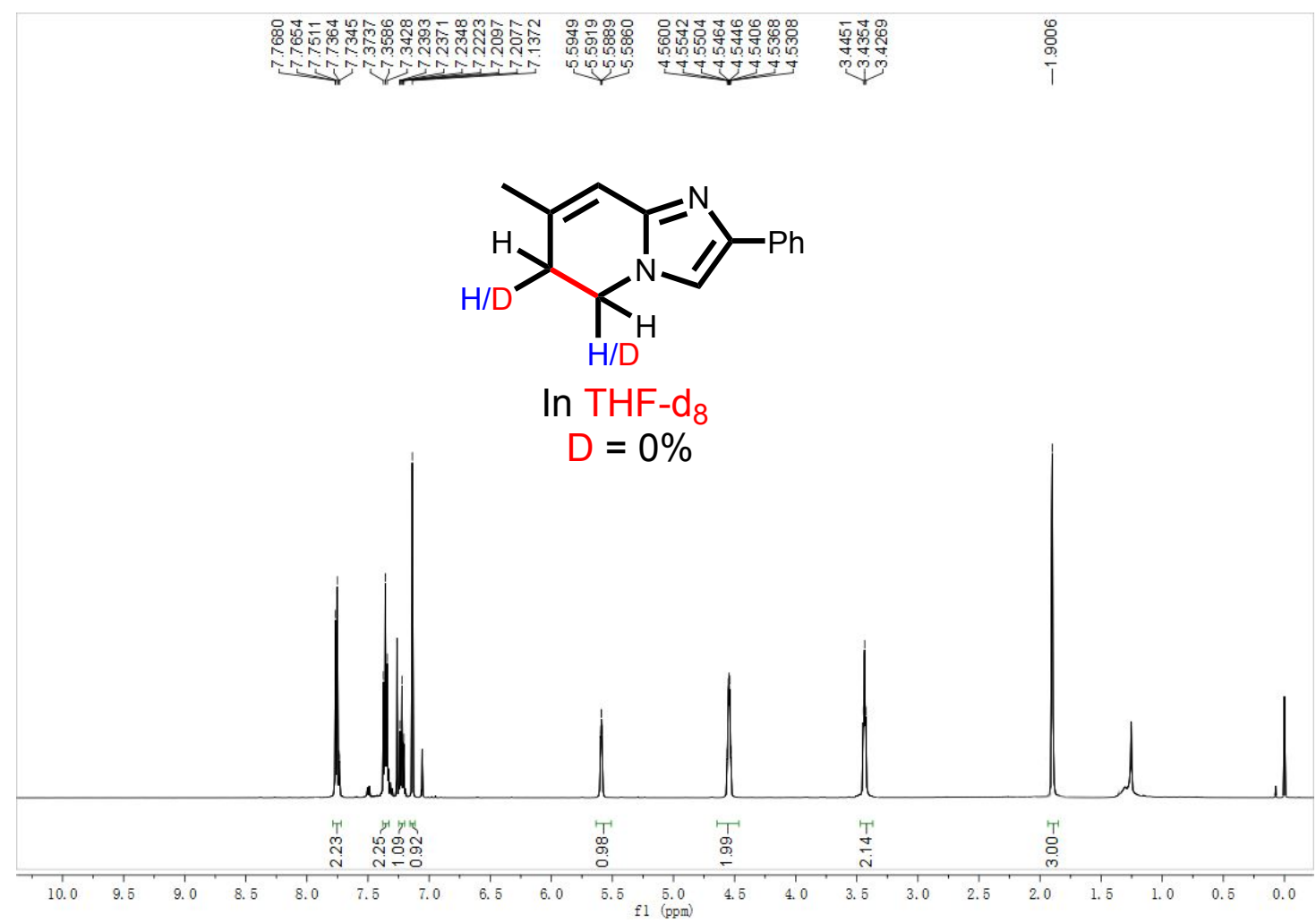

Fig. S3. Deuteration experiments results of $\mathrm{THF}-\mathrm{d}_{8}$

(2) Radical trapping experiments<smiles>Cc1ccn2cc(-c3ccccc3)nc2c1</smiles>

$1 b$

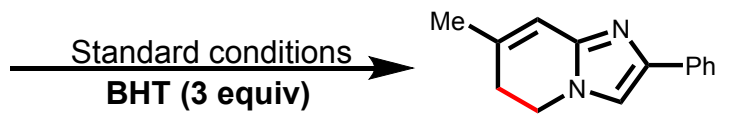

2b, trace<smiles>CCc1cc([N+](=O)[O-])cc(Br)c1OC1Cn2cc(-c3ccccc3)nc2C=C1C</smiles><smiles>Cc1ccn2cc(-c3ccccc3)nc2c1</smiles>

1b

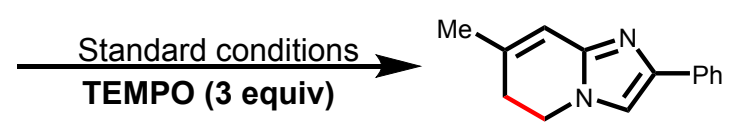

2b, n. d.

In an oven-dried undivided three-necked bottle $(25 \mathrm{~mL})$ equipped with a stir bar, ketene dithioacetals $\mathbf{1 b}(0.25 \mathrm{mmol}, 52.0 \mathrm{mg}),{ }^{\mathrm{n}} \mathrm{Bu}_{4} \mathrm{NBF}_{4}(1.0 \mathrm{mmol}, 330.0 \mathrm{mg})$, TEMPO or BHT $(0.75$ mmol) and $\mathrm{CH}_{3} \mathrm{CN} / \mathrm{THF}(9.0 / 1.0=10 \mathrm{~mL})$ were combined and sealed. The bottle was equipped with graphite rod as anode and platinum electrodes $\left(1 \times 1 \mathrm{~cm}^{2}\right)$ as cathode, and was then charged with nitrogen. The reaction mixture was stirred and electrolyzed at a constant current of $15 \mathrm{~mA}$ under room temperature for $4 \mathrm{~h}$. When the reaction was finished, the solution was concentrated in vacuum and the yield of $\mathbf{2 b}$ was sharply decreased. The intermediate $\mathbf{4}$ was detected by HRMS (ESI) calcd for $\mathrm{C}_{29} \mathrm{H}_{37} \mathrm{~N}_{2} \mathrm{O}+[\mathrm{M}+\mathrm{H}]^{+}:$429.2900; found: 429.2936). 


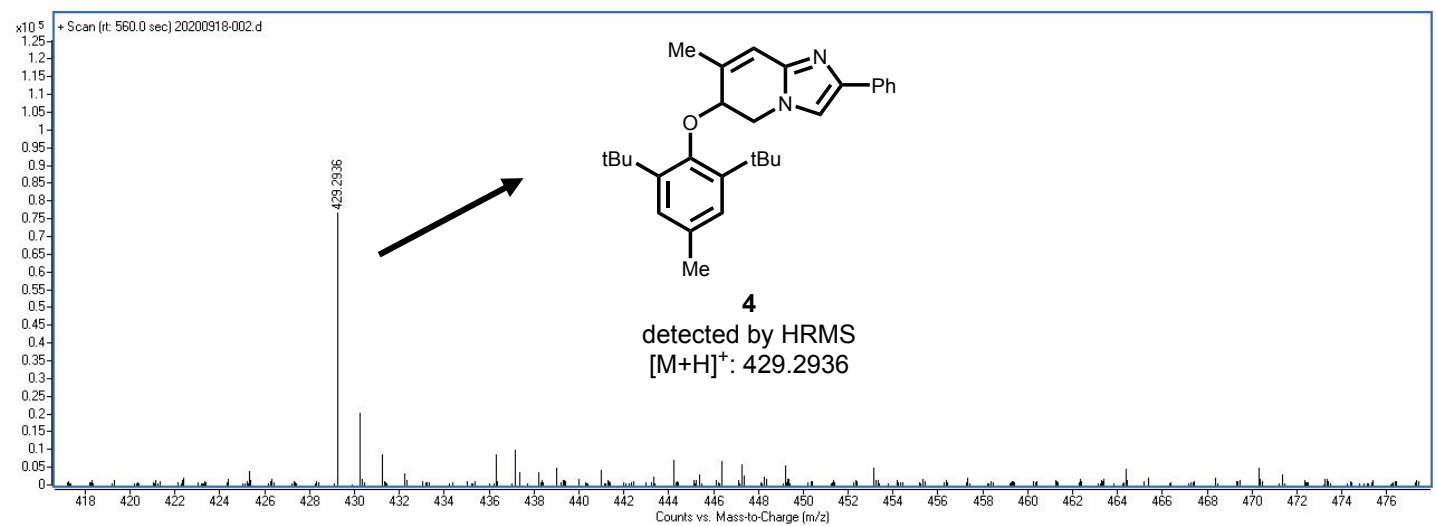

Fig. S4. The HRMS results of 4
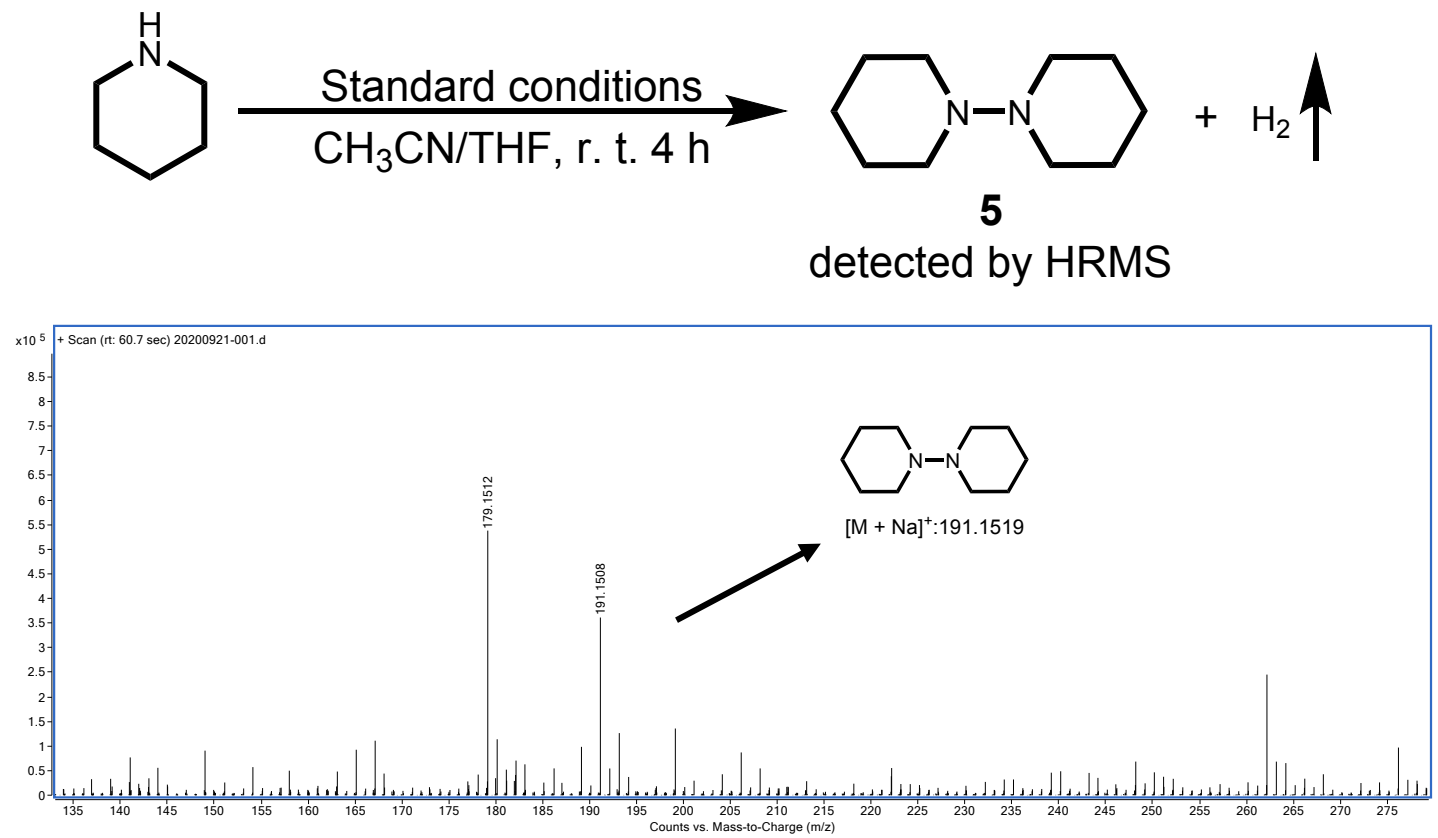

Fig. S5. The HRMS results of 5

(3) CV experiments
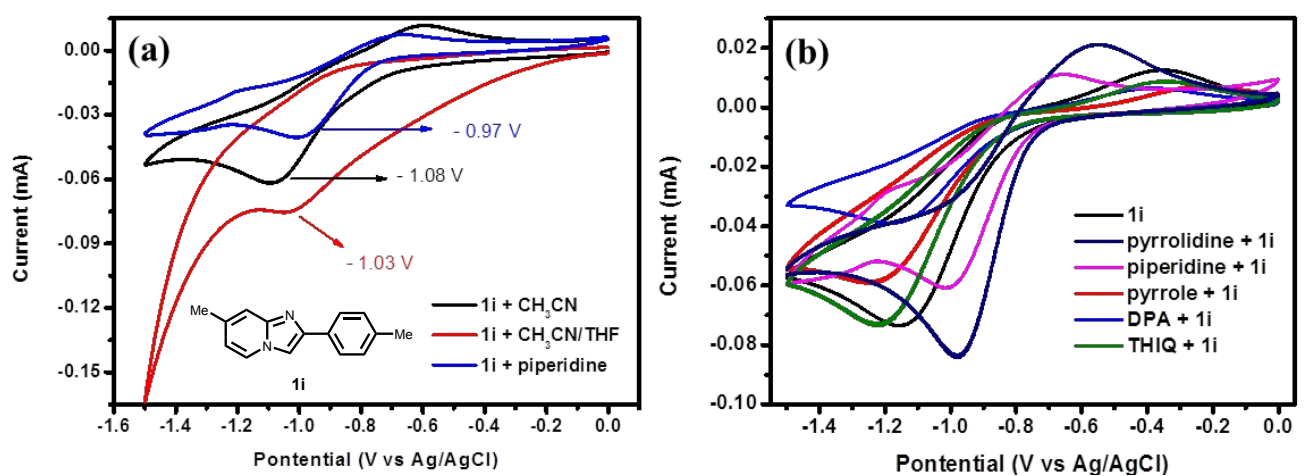

Fig. S6. Cyclic voltammograms at grass carbon as work electrode, $\mathrm{Pt}(1 \mathrm{x} 1 \mathrm{~cm})$ as counter electrode in 0.1 $\mathrm{M}^{n} \mathrm{Bu}_{4} \mathrm{NBF}_{4}, \mathbf{1 i}(0.25 \mathrm{mM})$, a: $\mathrm{CH}_{3} \mathrm{CN}+\mathrm{THF}(\mathrm{v}=9: 1)$, piperdine $(0.75 \mathrm{mM})$ in $\mathrm{CH}_{3} \mathrm{CN}$. b: pyrrolidine (0.75 $\mathrm{mM})$, piperdine $(0.75 \mathrm{mM})$, pyrrole $(0.75 \mathrm{mM})$, DPA: diphenylamine $(0.75 \mathrm{mM})$, THIQ: tetrahydroisoquinoline $(0.75 \mathrm{mM})$ in $\mathrm{CH}_{3} \mathrm{CN}$, scan rate $100 \mathrm{mV} / \mathrm{s}$. 


\section{References}

1. (a) Saldabol, N. O. and Giller, S. A., Chem. Heterocycl. Com., 1976, 12, 1155; (b) Cao, H., Zhan, H., Lin, Y., Lin, X., Du, Z. and Jiang, H., Org. Lett., 2012, 14, 1688; (c) Bagdi, A. K. and Hajra, A., The Chem. Rec., 2016, 16, 1868; (d) Samanta, S., Mondal, S., Santra, S., Kibriya, G. and Hajra, A., J. Org. Chem., 2016, 81, 10088; (e) Mondal, S., Samanta, S., Singsardar, M. and Hajra, A., Org. Lett., 2017, 19, 3751.

\section{Detail descriptions for products}

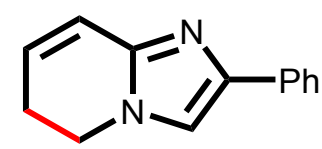

2-phenyl-5,6-dihydroimidazo[1,2-a]pyridine (2a): The masses of the starting materials $\mathbf{1 a}(0.25$ mmol, $48.5 \mathrm{mg}$ ), yellow liquid was obtained by column chromatography (eluent: EtOAc/petroleum ether $=1 / 10)$ with $76 \%$ isolated yield $(37.2 \mathrm{mg}) .{ }^{1} \mathrm{H} \mathrm{NMR}\left(500 \mathrm{MHz}, \mathrm{CDCl}_{3}\right) \delta$ $7.80-7.72(\mathrm{~m}, 2 \mathrm{H}), 7.36(\mathrm{t}, J=7.7 \mathrm{~Hz}, 2 \mathrm{H}), 7.22(\mathrm{t}, J=7.4 \mathrm{~Hz}, 1 \mathrm{H}), 7.13(\mathrm{~s}, 1 \mathrm{H}), 6.08-6.01(\mathrm{~m}$, 1H), $5.92-5.85(\mathrm{~m}, 1 \mathrm{H}), 4.59(\mathrm{~m}, 2 \mathrm{H}), 3.60-3.52(\mathrm{~m}, 2 \mathrm{H}) .{ }^{13} \mathrm{C} \mathrm{NMR}\left(126 \mathrm{MHz}, \mathrm{CDCl}_{3}\right) \delta 142.5$, 141.1, 134.2, 128.6, 126.7, 124.8, 123.1, 119.7, 113.1, 45.0, 25.1. HRMS (ESI) m/z: $[\mathrm{M}+\mathrm{H}]^{+}$calcd for $\mathrm{C}_{13} \mathrm{H}_{13} \mathrm{~N}_{2}$ 197.1073; found: 197.1071<smiles>CC1=Cc2nc(-c3ccccc3)cn2CC1</smiles>

7-methyl-2-phenyl-5,6-dihydroimidazo[1,2-a]pyridine (2b): The masses of the starting materials $\mathbf{1 b}(0.25 \mathrm{mmol}, 52.0 \mathrm{mg})$, white solid was obtained by column chromatography (eluent: EtOAc/petroleum ether $=1 / 7)$ with $92 \%$ isolated yield $(48.3 \mathrm{mg}) . \mathrm{m} . \mathrm{p} .=126-128^{\circ} \mathrm{C} .{ }^{1} \mathrm{H}$ NMR $\left(500 \mathrm{MHz}, \mathrm{CDCl}_{3}\right) \delta 7.78(\mathrm{~d}, J=7.5 \mathrm{~Hz}, 2 \mathrm{H}), 7.38(\mathrm{t}, J=7.6 \mathrm{~Hz}, 2 \mathrm{H}), 7.24(\mathrm{t}, J=7.3 \mathrm{~Hz}, 1 \mathrm{H}), 7.14(\mathrm{~s}$, 1H), $5.60(\mathrm{~s}, 1 \mathrm{H}), 4.54(\mathrm{~s}, 2 \mathrm{H}), 3.45(\mathrm{~s}, 2 \mathrm{H}), 1.91(\mathrm{~s}, 3 \mathrm{H}) .{ }^{13} \mathrm{C} \mathrm{NMR}\left(126 \mathrm{MHz}, \mathrm{CDCl}_{3}\right) \delta 143.1,130.9$, 130.6, 128.9, 128.8, 127.4, 125.0, 113.8, 113.2, 45.1, 28.9, 22.8. HRMS (ESI) m/z: $[\mathrm{M}+\mathrm{H}]^{+}$calcd for $\mathrm{C}_{14} \mathrm{H}_{15} \mathrm{~N}_{2} 211.1230$; found: 211.1230 .

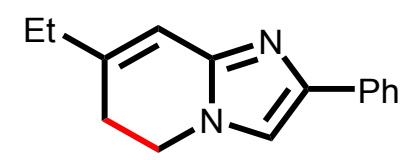

7-ethyl-2-phenyl-5,6-dihydroimidazo[1,2-a]pyridine (2c): The masses of the starting materials 1c $(0.25 \mathrm{mmol}, 55.5 \mathrm{mg})$, white liquid was obtained by column chromatography (eluent: EtOAc/petroleum ether $=1 / 7)$ with $83 \%$ isolated yield $(46.5 \mathrm{mg}) .{ }^{1} \mathrm{H} \mathrm{NMR}\left(500 \mathrm{MHz}, \mathrm{CDCl}_{3}\right) \delta$ 
$7.76(\mathrm{~d}, J=7.7 \mathrm{~Hz}, 2 \mathrm{H}), 7.36(\mathrm{t}, J=7.6 \mathrm{~Hz}, 2 \mathrm{H}), 7.22(\mathrm{t}, J=7.3 \mathrm{~Hz}, 1 \mathrm{H}), 7.14(\mathrm{~s}, 1 \mathrm{H}), 5.59(\mathrm{~s}, 1 \mathrm{H})$, $4.57(\mathrm{~d}, J=1.5 \mathrm{~Hz}, 2 \mathrm{H}), 3.46(\mathrm{~s}, 2 \mathrm{H}), 2.20(\mathrm{dd}, J=14.1,6.9 \mathrm{~Hz}, 2 \mathrm{H}), 1.13(\mathrm{t}, J=7.5 \mathrm{~Hz}, 3 \mathrm{H}) .{ }^{13} \mathrm{C}$ NMR $\left(126 \mathrm{MHz} \mathrm{CDCl}_{3}\right) \delta 143.4,141.2,136.5,134.1,128.6,126.7,124.8,112.9,112.4,45.0,29.5$, 27.9, 11.9. HRMS (ESI) $m / z:[\mathrm{M}+\mathrm{H}]^{+}$calcd for $\mathrm{C}_{15} \mathrm{H}_{17} \mathrm{~N}_{2}$ 225.1386; found: 225.1385 .

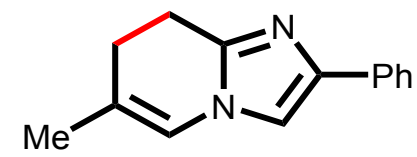

6-methyl-2-phenyl-7,8-dihydroimidazo[1,2-a]pyridine (2e): The masses of the starting materials 1e $(0.25 \mathrm{mmol}, 52.1 \mathrm{mg})$, white solid was obtained by column chromatography (eluent: EtOAc/petroleum ether $=1 / 7)$ with $96 \%$ isolated yield $(50.4 \mathrm{mg}) . \mathrm{m} . \mathrm{p} .=127-129^{\circ} \mathrm{C} .{ }^{1} \mathrm{H}$ NMR $\left(500 \mathrm{MHz}, \mathrm{CDCl}_{3}\right) \delta 7.69(\mathrm{~d}, J=7.4 \mathrm{~Hz}, 2 \mathrm{H}), 7.29(\mathrm{t}, J=7.4 \mathrm{~Hz}, 2 \mathrm{H}), 7.15(\mathrm{t}, J=7.2 \mathrm{~Hz}, 1 \mathrm{H}), 7.05(\mathrm{~s}$, 1H), $5.65(\mathrm{~s}, 1 \mathrm{H}), 4.37$ (s, 2H), $3.46(\mathrm{~s}, 2 \mathrm{H}), 1.78(\mathrm{~s}, 3 \mathrm{H}) .{ }^{13} \mathrm{C} \mathrm{NMR}\left(126 \mathrm{MHz}, \mathrm{CDCl}_{3}\right) \delta 142.7,141.3$, 134.4, 128.5, 127.0, 126.6, 124.7, 117.7, 112.8, 48.5, 25.1, 20.3. HRMS (ESI) m/z: $[\mathrm{M}+\mathrm{H}]{ }^{+}$calcd for $\mathrm{C}_{14} \mathrm{H}_{15} \mathrm{~N}_{2} 211.1230$; found: 211.1231 .

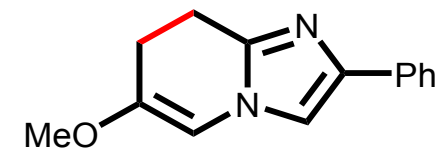

6-methoxy-2-phenyl-7,8-dihydroimidazo[1,2-a]pyridine (2f): The masses of the starting materials 1 (f $(0.25 \mathrm{mmol}, 56.0 \mathrm{mg})$, white solid was obtained by column chromatography (eluent: EtOAc/petroleum ether $=1 / 5)$ with $85 \%$ isolated yield $(48.0 \mathrm{mg}) . \mathrm{m} . \mathrm{p} .=148-151^{\circ} \mathrm{C} .{ }^{1} \mathrm{H}$ NMR $\left(500 \mathrm{MHz}, \mathrm{CDCl}_{3}\right) \delta 7.68(\mathrm{~d}, J=7.4 \mathrm{~Hz}, 2 \mathrm{H}), 7.28(\mathrm{t}, J=7.7 \mathrm{~Hz}, 2 \mathrm{H}), 7.15(\mathrm{t}, J=7.4 \mathrm{~Hz}, 1 \mathrm{H}), 7.05(\mathrm{~s}$, 1H), $4.88(\mathrm{~s}, 1 \mathrm{H}), 4.43(\mathrm{t}, J=3.9 \mathrm{~Hz}, 2 \mathrm{H}), 3.59(\mathrm{~s}, 3 \mathrm{H}), 3.52(\mathrm{dd}, J=8.2,4.1 \mathrm{~Hz}, 2 \mathrm{H}) .{ }^{13} \mathrm{C}$ NMR $(126$ $\left.\mathrm{MHz}, \mathrm{CDCl}_{3}\right) \delta 148.4,143.1,141.8,134.2,128.5,126.7,124.8,112.8,89.6,54.9,45.5,23.2 . \mathrm{HRMS}$ (ESI) $m / z:[\mathrm{M}+\mathrm{H}]{ }^{+}$calcd for $\mathrm{C}_{14} \mathrm{H}_{15} \mathrm{~N}_{2} \mathrm{O} 227.1179$; found: 227.1180 .

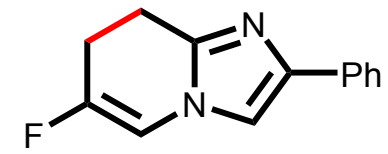

6-fluoro-2-phenyl-7,8-dihydroimidazo[1,2-a]pyridine (2g): The masses of the starting materials $1 \mathrm{~g}(0.25 \mathrm{mmol}, 53.0 \mathrm{mg})$, yellow oil was obtained by column chromatography (eluent: EtOAc/petroleum ether $=1 / 7)$ with $86 \%$ isolated yield $(46.0 \mathrm{mg}) . \mathrm{m} . \mathrm{p} .=150-153^{\circ} \mathrm{C} .{ }^{1} \mathrm{H}$ NMR $\left(500 \mathrm{MHz}, \mathrm{CDCl}_{3}\right) \delta 7.76-7.68(\mathrm{~m}, 2 \mathrm{H}), 7.36(\mathrm{t}, J=7.7 \mathrm{~Hz}, 2 \mathrm{H}), 7.26-7.21(\mathrm{~m}, 1 \mathrm{H}), 7.09(\mathrm{~s}, 1 \mathrm{H})$, $5.66-5.55(\mathrm{~m}, 1 \mathrm{H}), 4.60(\mathrm{t}, J=4.9 \mathrm{~Hz}, 2 \mathrm{H}), 3.62-3.53(\mathrm{~m}, 2 \mathrm{H}) .{ }^{13} \mathrm{C} \mathrm{NMR}\left(126 \mathrm{MHz}, \mathrm{CDCl}_{3}\right) \delta$ $151.5(\mathrm{~d}, 1 \mathrm{JC}-\mathrm{F}=248.9 \mathrm{~Hz}), 142.4,141.6,141.5,133.9,128.6,126.9,124.8,113.0,99.4(\mathrm{~d}, 3 \mathrm{JC}-\mathrm{F}=$ 
16.2 Hz), , $43.6(\mathrm{~d}, 2 \mathrm{JC}-\mathrm{F}=41.5 \mathrm{~Hz}), 22.7 .{ }^{19} \mathrm{~F} \mathrm{NMR}\left(471 \mathrm{MHz}, \mathrm{CDCl}_{3}\right) \delta-116.93 . \mathrm{HRMS}(\mathrm{ESI}) \mathrm{m} / \mathrm{z}$ : $[\mathrm{M}+\mathrm{H}]^{+}$calcd for $\mathrm{C}_{13} \mathrm{H}_{12} \mathrm{~N}_{2} \mathrm{~F} 215.0979$; found: 215.0980 .

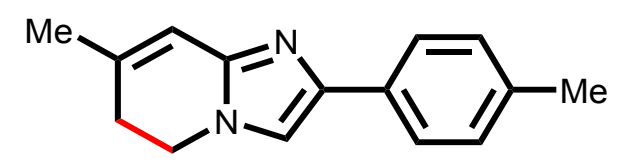

7-methyl-2-(p-tolyl)-5,6-dihydroimidazo[1,2-a]pyridine (2i): The masses of the starting materials $1 \mathbf{i}(0.25 \mathrm{mmol}, 55.5 \mathrm{mg})$, yellow liquid was obtained by column chromatography (eluent: EtOAc/petroleum ether $=1 / 7)$ with $78 \%$ isolated yield $(43.7 \mathrm{mg}) .{ }^{1} \mathrm{H} \mathrm{NMR}(500 \mathrm{MHz}$, $\left.\mathrm{CDCl}_{3}\right) \delta 7.65(\mathrm{~d}, J=8.1 \mathrm{~Hz}, 2 \mathrm{H}), 7.17(\mathrm{~d}, J=7.9 \mathrm{~Hz}, 2 \mathrm{H}), 7.10(\mathrm{~s}, 1 \mathrm{H}), 5.59(\mathrm{~d}, J=1.5 \mathrm{~Hz}, 1 \mathrm{H}), 4.62$ $-4.44(\mathrm{~m}, 2 \mathrm{H}), 3.43(\mathrm{t}, J=4.5 \mathrm{~Hz}, 2 \mathrm{H}), 2.35(\mathrm{~s}, 3 \mathrm{H}), 1.90(\mathrm{~s}, 3 \mathrm{H}) .{ }^{13} \mathrm{C} \mathrm{NMR}\left(126 \mathrm{MHz}, \mathrm{CDCl}_{3}\right) \delta$ 142.9, 141.4, 136.2, 131.5, 131.1, 129.2, 124.7, 114.1, 112.5, 44.9, 29.7, 22.9, 21.2. HRMS (ESI) m/z: $[\mathrm{M}+\mathrm{H}]^{+}$calcd for $\mathrm{C}_{15} \mathrm{H}_{17} \mathrm{~N}_{2} 225.1386$; found: 225.1388 .

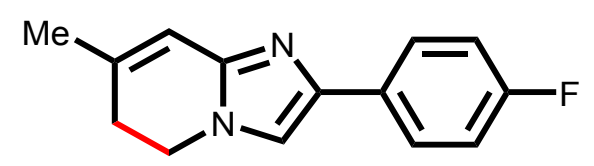

2-(4-fluorophenyl)-7-methyl-5,6-dihydroimidazo[1,2-a]pyridine (2j): The masses of the starting materials $\mathbf{1 j}$ (0.25 mmol, $56.5 \mathrm{mg})$, white solid was obtained by column chromatography (eluent: EtOAc/petroleum ether $=1 / 7)$ with $73 \%$ isolated yield $(41.6 \mathrm{mg}) . \mathrm{m} . \mathrm{p} .=127-130^{\circ} \mathrm{C} .{ }^{1} \mathrm{H}$ $\operatorname{NMR}\left(500 \mathrm{MHz}, \mathrm{CDCl}_{3}\right) \delta 7.77-7.68(\mathrm{~m}, 2 \mathrm{H}), 7.07(\mathrm{~s}, 1 \mathrm{H}), 7.04(\mathrm{t}, J=8.7 \mathrm{~Hz}, 2 \mathrm{H}), 5.59(\mathrm{~d}, J=1.3$ $\mathrm{Hz}, 1 \mathrm{H}), 4.57-4.51(\mathrm{~m}, 2 \mathrm{H}), 3.41(\mathrm{t}, J=4.7 \mathrm{~Hz}, 2 \mathrm{H}), 1.90(\mathrm{~s}, 3 \mathrm{H}) .{ }^{13} \mathrm{C} \mathrm{NMR}\left(126 \mathrm{MHz}, \mathrm{CDCl}_{3}\right) \delta$ $161.9(\mathrm{~d}, 1 \mathrm{JC}-\mathrm{F}=244.9 \mathrm{~Hz}), 143.2,140.5,131.1,130.6,128.5,126.3(\mathrm{~d}, 3 \mathrm{JC}-\mathrm{F}=7.8 \mathrm{~Hz}), 124.8,115.4$ $(\mathrm{d}, 2 \mathrm{JC}-\mathrm{F}=21.5 \mathrm{~Hz}), 114.1,112.7,44.9,29.6,22.9 .{ }^{19} \mathrm{~F}$ NMR $\left(471 \mathrm{MHz}, \mathrm{CDCl}_{3}\right) \delta-116.3 . \mathrm{HRMS}$ (ESI) $m / z:[\mathrm{M}+\mathrm{H}]^{+}$calcd for $\mathrm{C}_{14} \mathrm{H}_{14} \mathrm{~N}_{2} \mathrm{~F}$ 229.1136; found: 229.1136 .

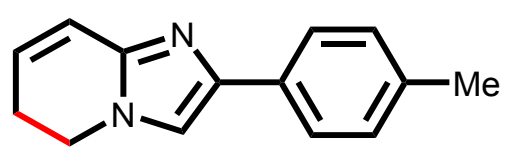

2-(p-tolyl)-5,6-dihydroimidazo[1,2-a]pyridine (2k): The masses of the starting materials $\mathbf{1 k}$ (0.25 mmol, $52.0 \mathrm{mg}$ ), white solid was obtained by column chromatography (eluent: EtOAc/petroleum ether $=1 / 7)$ with $70 \%$ isolated yield $(36.8 \mathrm{mg}) . \mathrm{m} . \mathrm{p} .=252-255^{\circ} \mathrm{C} .{ }^{1} \mathrm{H} \mathrm{NMR}$ $\left(500 \mathrm{MHz}, \mathrm{CDCl}_{3}\right) \delta 7.65(\mathrm{~d}, J=8.1 \mathrm{~Hz}, 2 \mathrm{H}), 7.17(\mathrm{~d}, J=7.9 \mathrm{~Hz}, 2 \mathrm{H}), 7.08(\mathrm{~s}, 1 \mathrm{H}), 6.06-6.01(\mathrm{~m}$, 1H), $5.91-5.85(\mathrm{~m}, 1 \mathrm{H}), 4.57(\mathrm{dq}, J=7.9,2.7 \mathrm{~Hz}, 2 \mathrm{H}), 3.59-3.51(\mathrm{~m}, 2 \mathrm{H}), 2.35(\mathrm{~s}, 3 \mathrm{H}) .{ }^{13} \mathrm{C}$ NMR $\left(126 \mathrm{MHz}, \mathrm{CDCl}_{3}\right) \delta 142.3,141.2,136.3,131.4,129.2,124.7,123.1,119.7,112.7,44.9,25.1,21.2$. HRMS (ESI) $m / z:[M+H]^{+}$calcd for $\mathrm{C}_{14} \mathrm{H}_{15} \mathrm{~N}_{2}$ 211.1230; found: 211.1230 . 


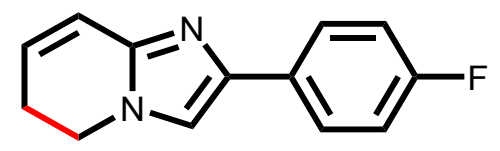

2-(4-fluorophenyl)-5,6-dihydroimidazo[1,2-a]pyridine (2l): The masses of the starting materials

11 (0.25 mmol, $53.0 \mathrm{mg}$ ),yellow oil was obtained by column chromatography (eluent: EtOAc/petroleum ether $=1 / 7)$ with $62 \%$ isolated yield $(33.2 \mathrm{mg}) .{ }^{1} \mathrm{H}$ NMR $\left(500 \mathrm{MHz}, \mathrm{CDCl}_{3}\right) \delta$ $8.17(\mathrm{~s}, 1 \mathrm{H}), 7.63(\mathrm{t}, J=7.4 \mathrm{~Hz}, 1 \mathrm{H}), 7.58(\mathrm{~d}, J=9.1 \mathrm{~Hz}, 1 \mathrm{H}), 7.33(\mathrm{dd}, J=13.6,6.7 \mathrm{~Hz}, 1 \mathrm{H}), 7.21-$ $7.18(\mathrm{~m}, 1 \mathrm{H}), 7.12(\mathrm{t}, J=7.8 \mathrm{~Hz}, 2 \mathrm{H}), 2.36(\mathrm{~s}, 3 \mathrm{H}), 2.18(\mathrm{~s}, 3 \mathrm{H}) .{ }^{13} \mathrm{C} \mathrm{NMR}\left(126 \mathrm{MHz}, \mathrm{CDCl}_{3}\right) \delta 161.9$ $(\mathrm{d}, 1 \mathrm{JC}-\mathrm{F}=245.0 \mathrm{~Hz}), 142.5,140.3,130.9,130.3,128.8,126.4(\mathrm{~d}, 3 \mathrm{JC}-\mathrm{F}=7.9 \mathrm{~Hz}), 123.0,119.6$, $115.4(\mathrm{~d}, 2 \mathrm{JC}-\mathrm{F}=21.5 \mathrm{~Hz}), 112.7,45.0,25.0 .{ }^{19} \mathrm{~F}$ NMR $\left(471 \mathrm{MHz}, \mathrm{CDCl}_{3}\right) \delta-116.0$. HRMS (ESI) m/z: $[\mathrm{M}+\mathrm{H}]^{+}$calcd for $\mathrm{C}_{13} \mathrm{H}_{12} \mathrm{~N}_{2} \mathrm{~F}$ 215.0979; found: 215.0980.

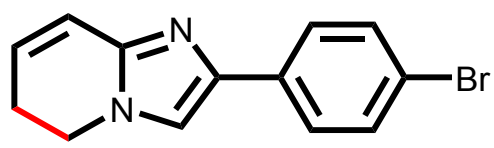

2-(4-bromophenyl)-5,6-dihydroimidazo[1,2-a]pyridine (2m): The masses of the starting materials $1 \mathrm{~m}(0.25 \mathrm{mmol}, 68.0 \mathrm{mg})$, yellow oil was obtained by column chromatography (eluent: EtOAc/petroleum ether $=1 / 7)$ with $65 \%$ isolated yield $(44.5 \mathrm{mg}) .{ }^{1} \mathrm{H} \mathrm{NMR}\left(500 \mathrm{MHz}, \mathrm{CDCl}_{3}\right) \delta$ $7.76(\mathrm{~d}, J=7.5 \mathrm{~Hz}, 2 \mathrm{H}), 7.36(\mathrm{t}, J=7.6 \mathrm{~Hz}, 2 \mathrm{H}), 7.23(\mathrm{t}, J=7.4 \mathrm{~Hz}, 1 \mathrm{H}), 7.15(\mathrm{~s}, 1 \mathrm{H}), 6.12-6.01(\mathrm{~m}$, 1H), $5.93-5.85(\mathrm{~m}, 1 \mathrm{H}), 4.70-4.51(\mathrm{~m}, 2 \mathrm{H}), 3.61-3.49(\mathrm{~m}, 2 \mathrm{H}) .{ }^{13} \mathrm{C} \mathrm{NMR}\left(126 \mathrm{MHz}, \mathrm{CDCl}_{3}\right) \delta$ 142.6, 141.0, 134.0, 128.6, 126.7, 124.8, 123.0, 119.6, 113.1, 45.0, 25.0. HRMS (ESI) $m / z:[\mathrm{M}+\mathrm{H}]^{+}$ calcd for $\mathrm{C}_{13} \mathrm{H}_{12} \mathrm{~N}_{2} \mathrm{Br}$ 275.0178; found: 275. 0178 .

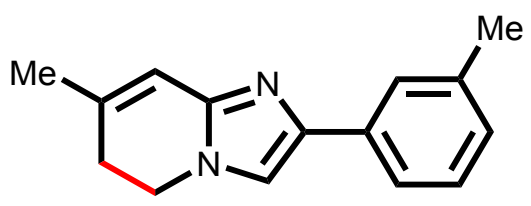

7-methyl-2-(m-tolyl)-5,6-dihydroimidazo[1,2-a]pyridine (2n): The masses of the starting materials 1 n $(0.25 \mathrm{mmol}, 55.5 \mathrm{mg})$, yellow solid was obtained by column chromatography (eluent: EtOAc/petroleum ether $=1 / 7)$ with $74 \%$ isolated yield $(41.4 \mathrm{mg}) . \mathrm{m} . \mathrm{p} .=255-257^{\circ} \mathrm{C} .{ }^{1} \mathrm{H}$ NMR $\left(500 \mathrm{MHz}, \mathrm{CDCl}_{3}\right) \delta 7.64(\mathrm{~s}, 1 \mathrm{H}), 7.52(\mathrm{~d}, J=7.7 \mathrm{~Hz}, 1 \mathrm{H}), 7.26-7.22(\mathrm{~m}, 1 \mathrm{H}), 7.13(\mathrm{~s}, 1 \mathrm{H}), 7.04(\mathrm{~d}, J$ $=8.3 \mathrm{~Hz}, 1 \mathrm{H}), 5.59(\mathrm{~s}, 1 \mathrm{H}), 4.56-4.54(\mathrm{~m}, 1 \mathrm{H}), 3.43(\mathrm{t}, J=4.6 \mathrm{~Hz}, 2 \mathrm{H}), 2.38(\mathrm{~s}, 3 \mathrm{H}), 1.90(\mathrm{~s}, 3 \mathrm{H}) .{ }^{13} \mathrm{C}$ NMR $\left(126 \mathrm{MHz}, \mathrm{CDCl}_{3}\right) \delta 143.0,141.3,138.1,134.0,131.1,128.4,127.5,125.5,121.9,114.1,112.9$, 44.9, 29.6, 22.9, 21.5. HRMS (ESI) $\mathrm{m} / z$ : $[\mathrm{M}+\mathrm{H}]^{+}$calcd for $\mathrm{C}_{15} \mathrm{H}_{17} \mathrm{~N}_{2}$ 225.1386; found: 225.1387 . 


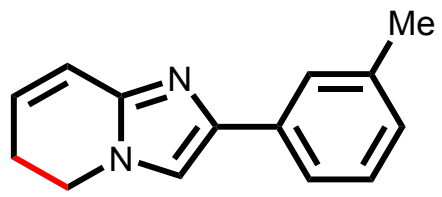

2-(m-tolyl)-5,6-dihydroimidazo[1,2-a]pyridine (20): The masses of the starting materials 10 (0.25 mmol, $52.0 \mathrm{mg}$ ), yellow oil was obtained by column chromatography (eluent: EtOAc/petroleum ether $=1 / 7)$ with $71 \%$ isolated yield $(37.3 \mathrm{mg}) . \mathrm{m} . \mathrm{p} .=251-253^{\circ} \mathrm{C} .{ }^{1} \mathrm{H}$ NMR $\left(500 \mathrm{MHz}, \mathrm{CDCl}_{3}\right) \delta 7.64(\mathrm{~s}, 1 \mathrm{H}), 7.52(\mathrm{~d}, J=7.7 \mathrm{~Hz}, 1 \mathrm{H}), 7.28-7.22(\mathrm{~m}, 1 \mathrm{H}), 7.13(\mathrm{~s}, 1 \mathrm{H}), 7.04(\mathrm{~d}, J$ $=7.5 \mathrm{~Hz}, 1 \mathrm{H}), 6.08-6.02(\mathrm{~m}, 1 \mathrm{H}), 5.92-5.86(\mathrm{~m}, 1 \mathrm{H}), 4.64-4.56(\mathrm{~m}, 2 \mathrm{H}), 3.60-3.53(\mathrm{~m}, 2 \mathrm{H}), 2.38$ (s, 3H). ${ }^{13} \mathrm{C}$ NMR $\left(126 \mathrm{MHz}, \mathrm{CDCl}_{3}\right) \delta 142.4,141.2,138.2,134.1,128.4,127.5,125.5,123.1,121.8$, 119.7, 113.1, 45.0, 25.2, 21.5. HRMS (ESI) $\mathrm{m} / \mathrm{z}$ : $[\mathrm{M}+\mathrm{H}]{ }^{+}$calcd for $\mathrm{C}_{14} \mathrm{H}_{15} \mathrm{~N}_{2}$ 211.1230; found: 211.1230<smiles>Fc1ccccc1-c1cn2c(n1)C=CCC2</smiles>

2-(2-fluorophenyl)-5,6-dihydroimidazo[1,2-a]pyridine (2p): The masses of the starting materials 1p (0.25 mmol, $53.0 \mathrm{mg})$, yellow liquid was obtained by column chromatography (eluent: EtOAc/petroleum ether $=1 / 7)$ with $81 \%$ isolated yield $(43.3 \mathrm{mg}) .{ }^{1} \mathrm{H} \mathrm{NMR}(500 \mathrm{MHz}$, $\left.\mathrm{CDCl}_{3}\right) \delta 8.13(\mathrm{~s}, 1 \mathrm{H}), 7.34(\mathrm{~d}, J=3.7 \mathrm{~Hz}, 1 \mathrm{H}), 7.26(\mathrm{~s}, 1 \mathrm{H}), 7.19(\mathrm{~s}, 1 \mathrm{H}), 7.15-7.01(\mathrm{~m}, 1 \mathrm{H}), 6.06(\mathrm{~d}$, $J=10.0 \mathrm{~Hz}, 1 \mathrm{H}), 5.91(\mathrm{~d}, J=10.2 \mathrm{~Hz}, 1 \mathrm{H}), 4.63(\mathrm{~s}, 2 \mathrm{H}), 3.58(\mathrm{~s}, 2 \mathrm{H}) .{ }^{13} \mathrm{C} \mathrm{NMR}\left(126 \mathrm{MHz}, \mathrm{CDCl}_{3}\right) \delta$ $159.6(\mathrm{~d}, 1 \mathrm{JC}-\mathrm{F}=247.5 \mathrm{~Hz}), 142.0,134.4,127.5(\mathrm{~d}, 3 \mathrm{JC}-\mathrm{F}=8.2 \mathrm{~Hz}), 124.3,124.3,123.0,119.7$, 117.5, 117.4, $115.4(\mathrm{~d}, 2 \mathrm{JC}-\mathrm{F}=22.0 \mathrm{~Hz}), 45.0,24.9 .{ }^{19} \mathrm{~F}$ NMR $\left(471 \mathrm{MHz}, \mathrm{CDCl}_{3}\right) \delta-114.5 . \mathrm{HRMS}$ (ESI) $m / z:[\mathrm{M}+\mathrm{H}]^{+}$calcd for $\mathrm{C}_{13} \mathrm{H}_{12} \mathrm{~N}_{2} \mathrm{~F} 2$ 215.0979; found: 215.0980 .

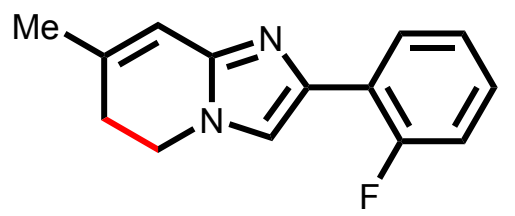

2-(2-fluorophenyl)-7-methyl-5,6-dihydroimidazo[1,2-a]pyridine (2q): The masses of the starting materials $1 q(0.25 \mathrm{mmol}, 56.5 \mathrm{mg})$, yellow solid was obtained by column chromatography (eluent: EtOAc/petroleum ether $=1 / 6)$ with $84 \%$ isolated yield $(47.9 \mathrm{mg}) . \mathrm{m} . \mathrm{p} .=108-110^{\circ} \mathrm{C} .{ }^{1} \mathrm{H}$ NMR $\left(500 \mathrm{MHz}, \mathrm{CDCl}_{3}\right) \delta 8.16-8.08(\mathrm{~m}, 1 \mathrm{H}), 7.33(\mathrm{~d}, J=4.1 \mathrm{~Hz}, 1 \mathrm{H}), 7.21-7.14(\mathrm{~m}, 2 \mathrm{H}), 5.59(\mathrm{~d}$, $J=1.4 \mathrm{~Hz}, 1 \mathrm{H}), 4.60-4.52(\mathrm{~m}, 2 \mathrm{H}), 3.43(\mathrm{t}, J=4.4 \mathrm{~Hz}, 2 \mathrm{H}), 1.90(\mathrm{~s}, 3 \mathrm{H}) .{ }^{13} \mathrm{C}$ NMR $(126 \mathrm{MHz}$, $\left.\mathrm{CDCl}_{3}\right) \delta 159.6(\mathrm{~d}, 1 \mathrm{JC}-\mathrm{F}=247.7 \mathrm{~Hz}), 142.7,134.5,130.9,127.5,127.4,127.4,124.3(\mathrm{~d}, 3 \mathrm{JC}-\mathrm{F}=8.2$ 
$\mathrm{Hz}), 117.4,115.4(\mathrm{~d}, 2 \mathrm{JC}-\mathrm{F}=22.0 \mathrm{~Hz}), 114.1,44.9,29.5,22.8 .{ }^{19} \mathrm{~F}$ NMR $\left(471 \mathrm{MHz}, \mathrm{CDCl}_{3}\right) \delta-114.5$. HRMS (ESI) m/z: $[\mathrm{M}+\mathrm{H}]^{+}$calcd for $\mathrm{C}_{14} \mathrm{H}_{14} \mathrm{~N}_{2} \mathrm{~F} 229.1136$; found: 229.1136 .

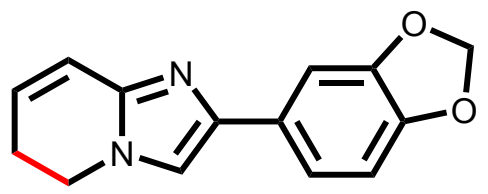

2-(benzo[d][1,3]dioxol-5-yl)-5,6-dihydroimidazo[1,2-a]pyridine (2r): The masses of the starting materials $1 \mathbf{r}(0.25 \mathrm{mmol}, 59.5 \mathrm{mg})$, yellow solid was obtained by column chromatography (eluent: EtOAc/petroleum ether $=1 / 4)$ with $71 \%$ isolated yield $(42.6 \mathrm{mg}) . \mathrm{m} . \mathrm{p} .=145-148^{\circ} \mathrm{C} .{ }^{1} \mathrm{H}$ NMR $\left(500 \mathrm{MHz}, \mathrm{CDCl}_{3}\right) \delta 7.28-7.23(\mathrm{~m}, 2 \mathrm{H}), 7.02(\mathrm{~s}, 1 \mathrm{H}), 6.81(\mathrm{~d}, J=8.0 \mathrm{~Hz}, 1 \mathrm{H}), 6.07-6.01(\mathrm{~m}$, 1H), $5.95(\mathrm{~s}, 2 \mathrm{H}), 5.92-5.87(\mathrm{~m}, 1 \mathrm{H}), 4.59-4.56(\mathrm{~m}, 2 \mathrm{H}), 3.57-3.50(\mathrm{~m}, 2 \mathrm{H}) .{ }^{13} \mathrm{C} \mathrm{NMR}(126 \mathrm{MHz}$, $\left.\mathrm{CDCl}_{3}\right) \delta 147.9,146.5,142.2,140.9,128.7,123.1,119.7,118.2,112.3,108.5,105.6,100.9,44.9,25.1$. HRMS (ESI) m/z: [M+H] ${ }^{+}$calcd for $\mathrm{C}_{14} \mathrm{H}_{13} \mathrm{~N}_{2} \mathrm{O}_{2}$ 241.0972; found: 241.0973 .

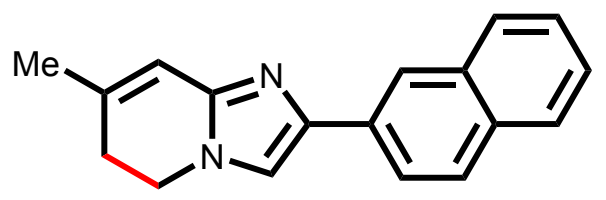

7-methyl-2-(naphthalen-2-yl)-5,6-dihydroimidazo[1,2-a]pyridine (2s): The masses of the starting materials $1 \mathrm{~s}(0.25 \mathrm{mmol}, 64.5 \mathrm{mg})$, yellow solid was obtained by column chromatography (eluent: EtOAc/petroleum ether $=1 / 7)$ with $75 \%$ isolated yield $(48.8 \mathrm{mg}) . \mathrm{m} . \mathrm{p} .=150-153^{\circ} \mathrm{C} .{ }^{1} \mathrm{H}$ $\operatorname{NMR}\left(500 \mathrm{MHz}, \mathrm{CDCl}_{3}\right) \delta 8.30(\mathrm{~s}, 1 \mathrm{H}), 7.89-7.76(\mathrm{~m}, 4 \mathrm{H}), 7.43(\mathrm{dt}, J=14.8,6.8 \mathrm{~Hz}, 2 \mathrm{H}), 7.26(\mathrm{~d}, J$ $=3.7 \mathrm{~Hz}, 1 \mathrm{H}), 5.62(\mathrm{~s}, 1 \mathrm{H}), 4.59(\mathrm{~s}, 2 \mathrm{H}), 3.48(\mathrm{~s}, 2 \mathrm{H}), 1.92(\mathrm{~s}, 3 \mathrm{H}) .{ }^{13} \mathrm{C} \mathrm{NMR}\left(126 \mathrm{MHz}, \mathrm{CDCl}_{3}\right) \delta$ 128.1, 127.6, 126.1, 123.6, 122.9, 113.9, 113.6, 45.1, 29.5, 22.9. HRMS (ESI) $m / z:[M+H]^{+}$calcd for $\mathrm{C}_{18} \mathrm{H}_{17} \mathrm{~N}_{2}$ 261.1386; found: 261.1389 .

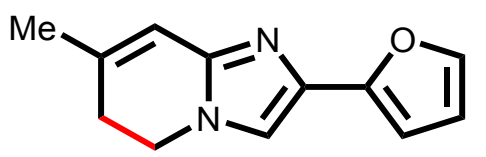

2-(furan-2-yl)-7-methyl-5,6-dihydroimidazo[1,2-a]pyridine (2t): The masses of the starting materials 1t $(0.25 \mathrm{mmol}, 49.5 \mathrm{mg})$, yellow liquid was obtained by column chromatography (eluent: EtOAc/petroleum ether $=1 / 5)$ with $73 \%$ isolated yield $(36.5 \mathrm{mg}) .{ }^{1} \mathrm{H}$ NMR $(500 \mathrm{MHz}$, $\left.\mathrm{CDCl}_{3}\right) \delta 7.37(\mathrm{~d}, J=1.1 \mathrm{~Hz}, 1 \mathrm{H}), 7.08(\mathrm{~s}, 1 \mathrm{H}), 6.61(\mathrm{~d}, J=3.3 \mathrm{~Hz}, 1 \mathrm{H}), 6.43(\mathrm{dd}, J=3.2,1.8 \mathrm{~Hz}, 1 \mathrm{H})$, $5.64-5.54(\mathrm{~m}, 1 \mathrm{H}), 4.59-4.47(\mathrm{~m}, 2 \mathrm{H}), 3.40(\mathrm{t}, J=4.6 \mathrm{~Hz}, 2 \mathrm{H}), 1.90(\mathrm{~s}, 3 \mathrm{H}) .{ }^{13} \mathrm{C}$ NMR $(126 \mathrm{MHz}$, $\left.\mathrm{CDCl}_{3}\right) \delta 150.1,143.2,140.8,133.8,131.0,114.0,112.9,111.2,103.9,45.0,29.5,22.8$. HRMS (ESI) 
$\mathrm{m} / \mathrm{z}:[\mathrm{M}+\mathrm{H}]^{+}$calcd for $\mathrm{C}_{12} \mathrm{H}_{13} \mathrm{~N}_{2} \mathrm{O}$ 201.1022; found: 201.1023.

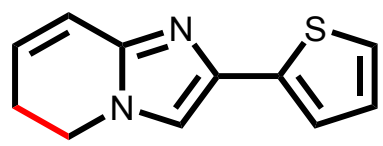

2-(thiophen-2-yl)-5,6-dihydroimidazo[1,2-a]pyridine (2u): The masses of the starting materials 1u $(0.25 \mathrm{mmol}, 50.0 \mathrm{mg})$, yellow liquid was obtained by column chromatography (eluent: EtOAc/petroleum ether $=1 / 5)$ with $77 \%$ isolated yield $(38.9 \mathrm{mg}) .{ }^{1} \mathrm{H}$ NMR $\left(500 \mathrm{MHz}, \mathrm{CDCl}_{3}\right) \delta$ $7.31-7.22(\mathrm{~m}, 1 \mathrm{H}), 7.17(\mathrm{~d}, J=4.8 \mathrm{~Hz}, 1 \mathrm{H}), 7.05(\mathrm{~s}, 1 \mathrm{H}), 7.03-7.00(\mathrm{~m}, 1 \mathrm{H}), 6.04(\mathrm{~d}, J=8.1 \mathrm{~Hz}$, 1H), $5.98-5.85(\mathrm{~m}, 1 \mathrm{H}), 4.69-4.53(\mathrm{~m}, 2 \mathrm{H}), 3.54(\mathrm{~m}, 2 \mathrm{H}) .{ }^{13} \mathrm{C} \mathrm{NMR}\left(126 \mathrm{MHz}, \mathrm{CDCl}_{3}\right) \delta 142.4$, $137.7,136.1,127.5,123.2,122.9,121.9,119.5,112.6,45.0,24.9$. HRMS (ESI) $m / z:[\mathrm{M}+\mathrm{H}]{ }^{+}$calcd for $\mathrm{C}_{11} \mathrm{H}_{11} \mathrm{~N}_{2} \mathrm{~S}$ 203.0637; found: 203.0635. 
8. Copies of product NMR and HRMS Spectra

$2 a$

${ }^{1}$ HNMR
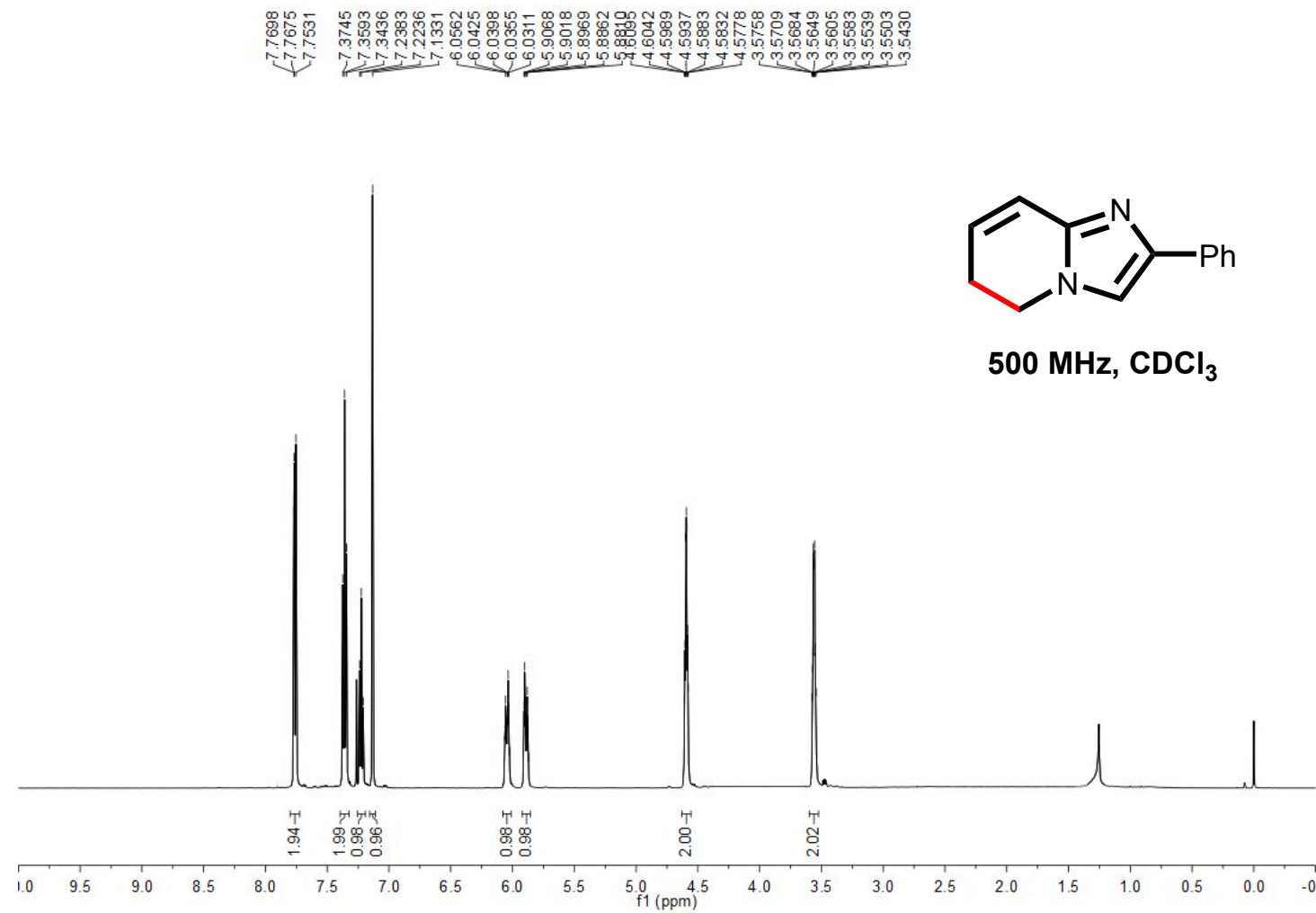

${ }^{13}$ CNMR

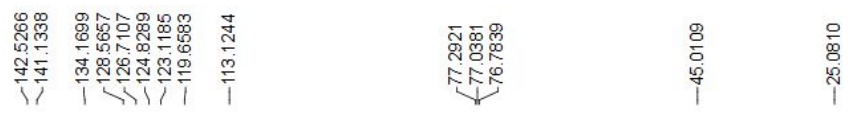

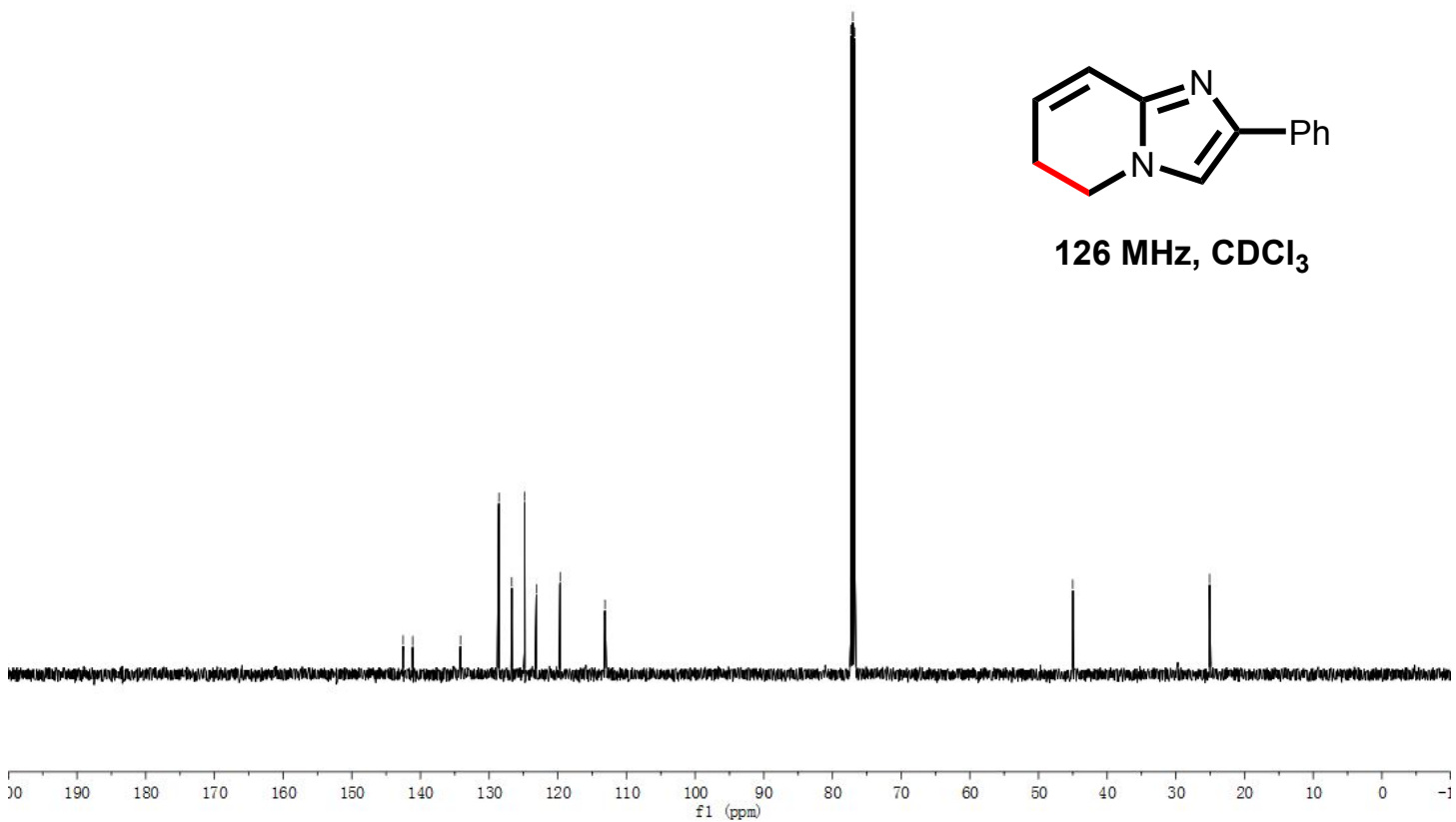


WJW-1\#10-23 RT: 0.05-0.11 AV: 14 SB: 276 0.71-1.28, 1.31-1.97 NL: $1.36 E 10$ T: FTMS + p ESI Full ms [100.0000-1000.0000]

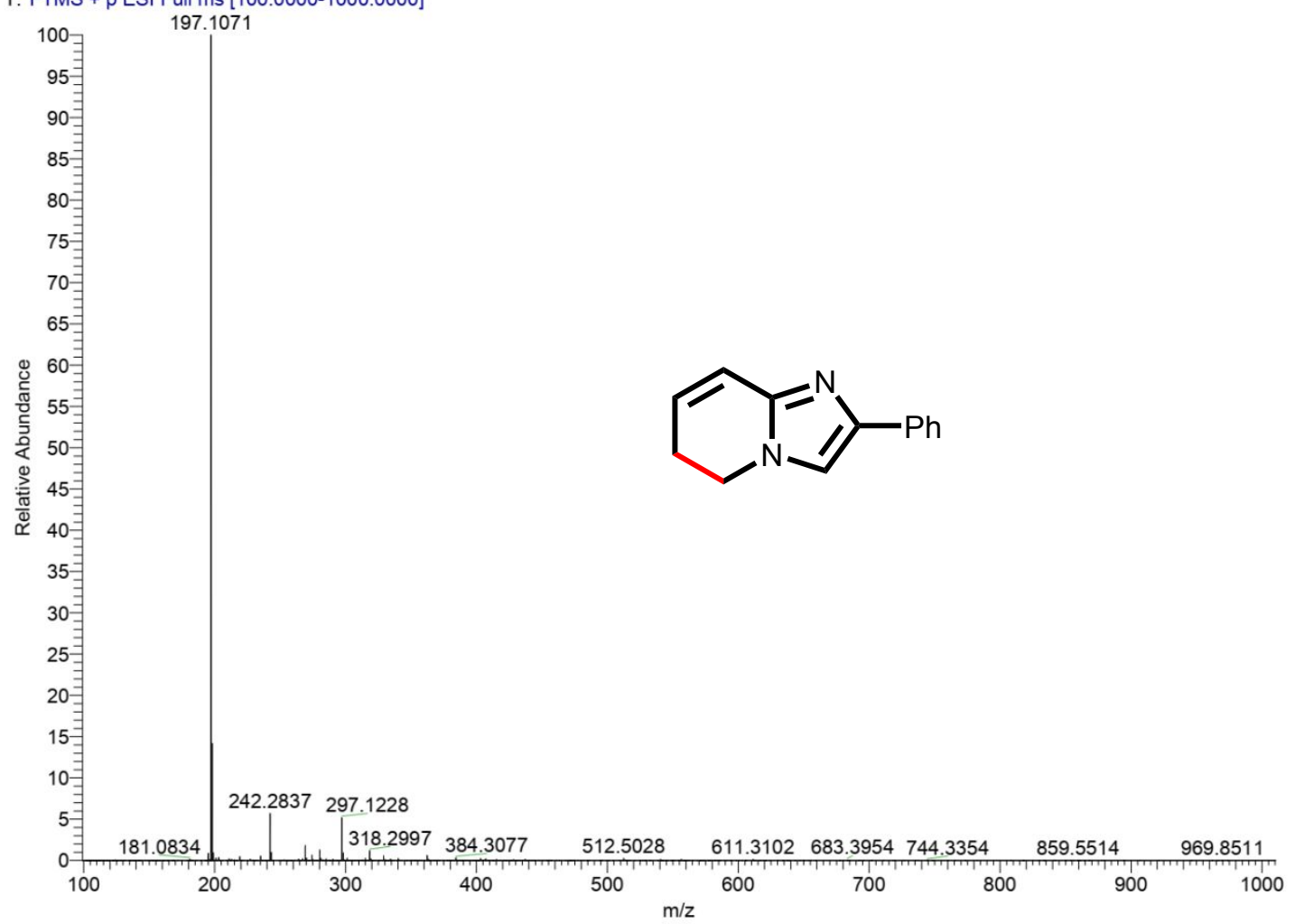


${ }^{1}$ HNMR

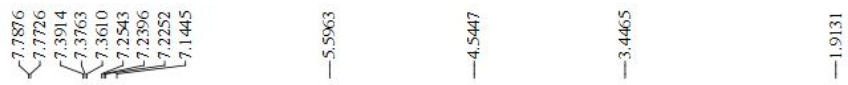<smiles>CC1=Cc2nc(-c3ccccc3)cn2CC1</smiles>

$500 \mathrm{MHz} \mathrm{CDCl}_{3}$

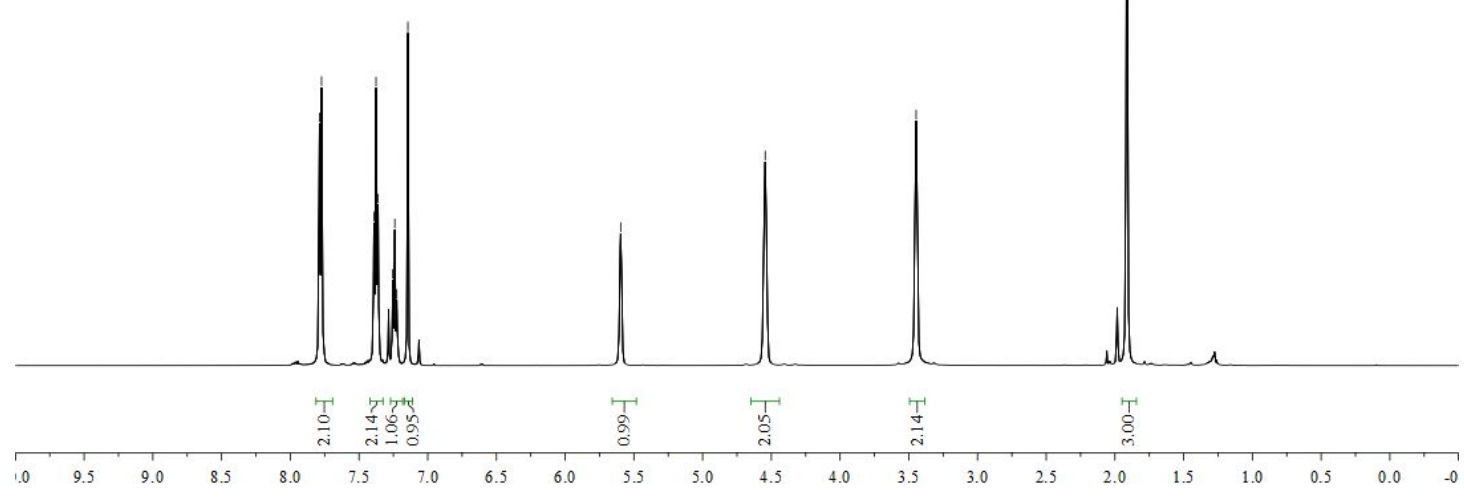

${ }^{13}$ CNMR

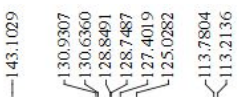

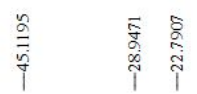<smiles>CC1=Cc2nc(-c3ccccc3)cn2CC1</smiles>

$126 \mathrm{MHz}^{\mathrm{CDCl}_{3}}$

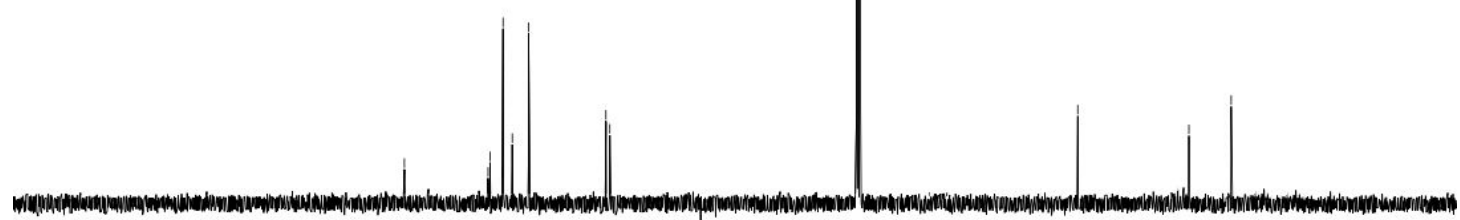


WJW-2 \#13-24 RT: 0.05-0.10 AV: 12 SB: 243 0.77-1.31, 1.40-1.94 NL: $1.02 E 10$ T: FTMS + p ESI Full ms [100.0000-1000.0000]

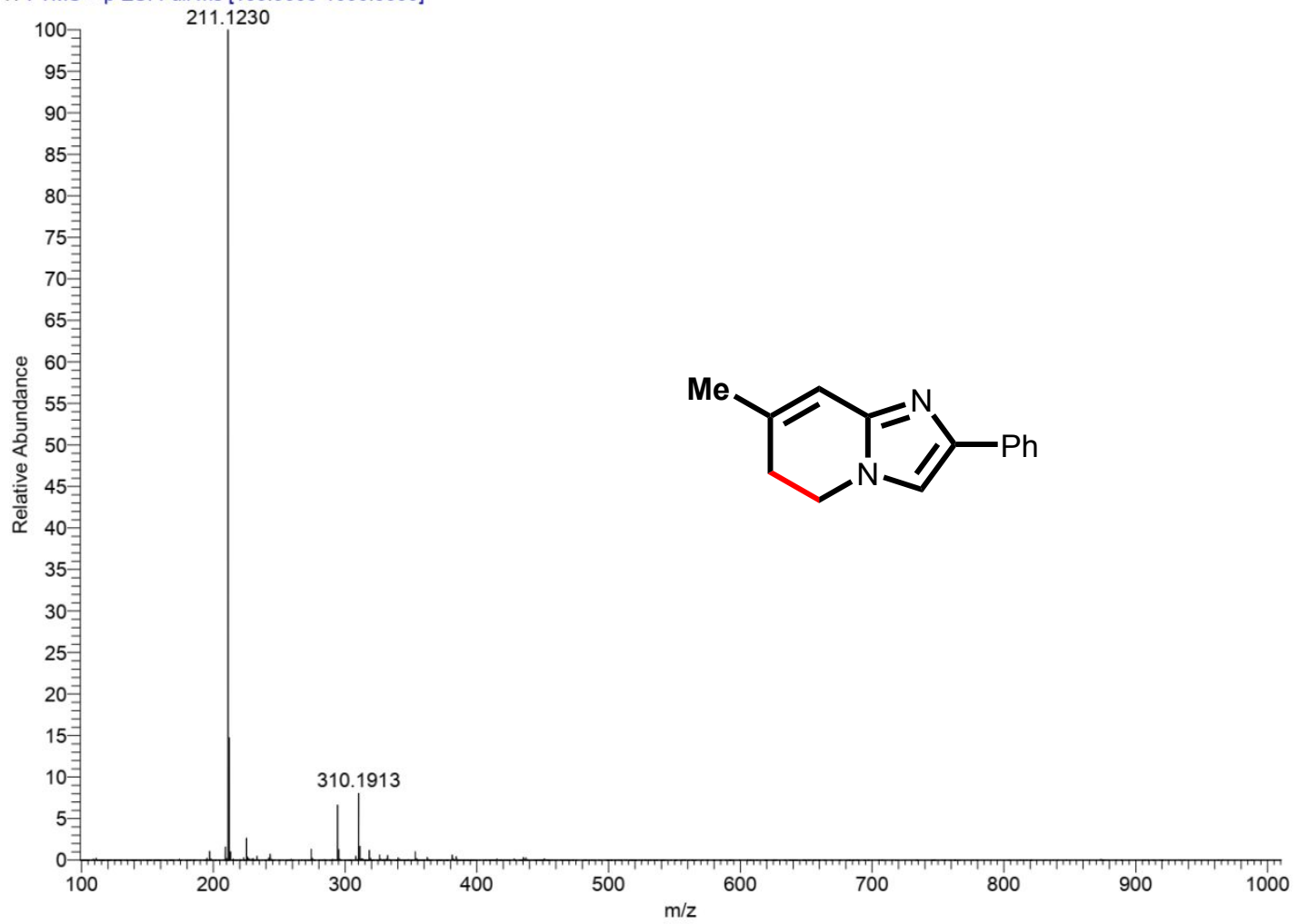


${ }^{1}$ HNMR

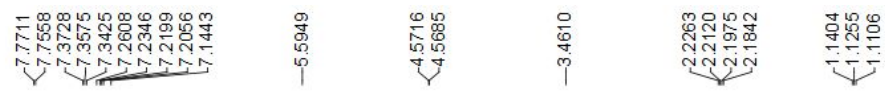<smiles>CCC1=Cc2nc(-c3ccccc3)cn2CC1</smiles>

$500 \mathrm{MHz}, \mathrm{CDCl}_{3}$

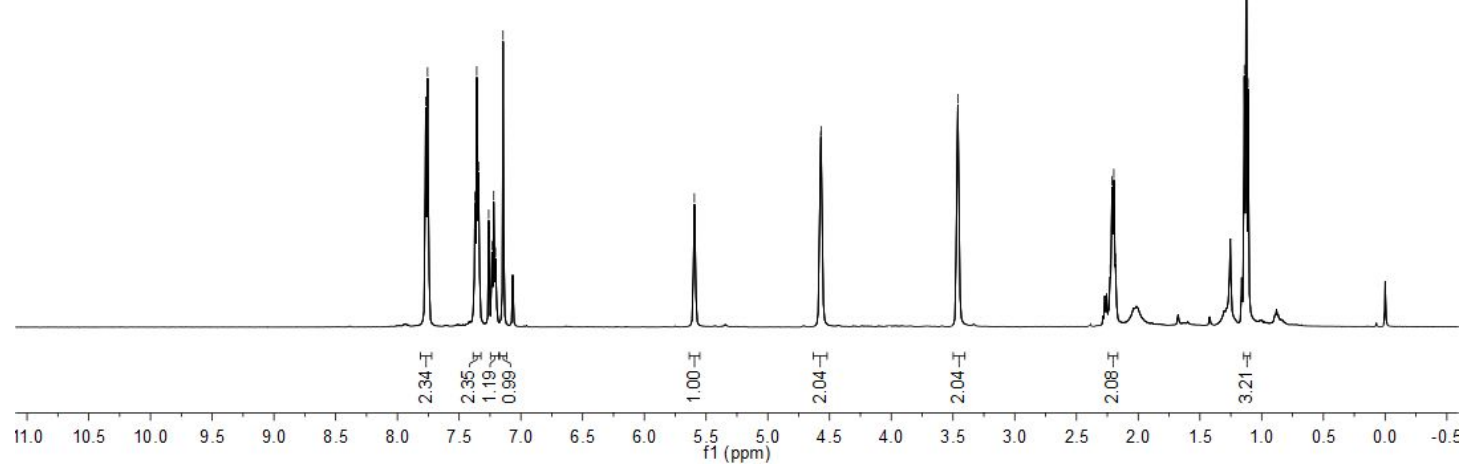

${ }^{13}$ CNMR

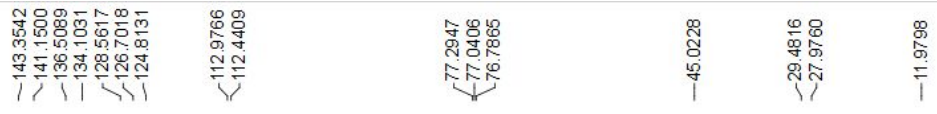

$\mathrm{CO}^{\mathrm{Et}} \mathrm{Ph}^{\mathrm{N}}$

$126 \mathrm{MHz}, \mathrm{CDCl}_{3}$

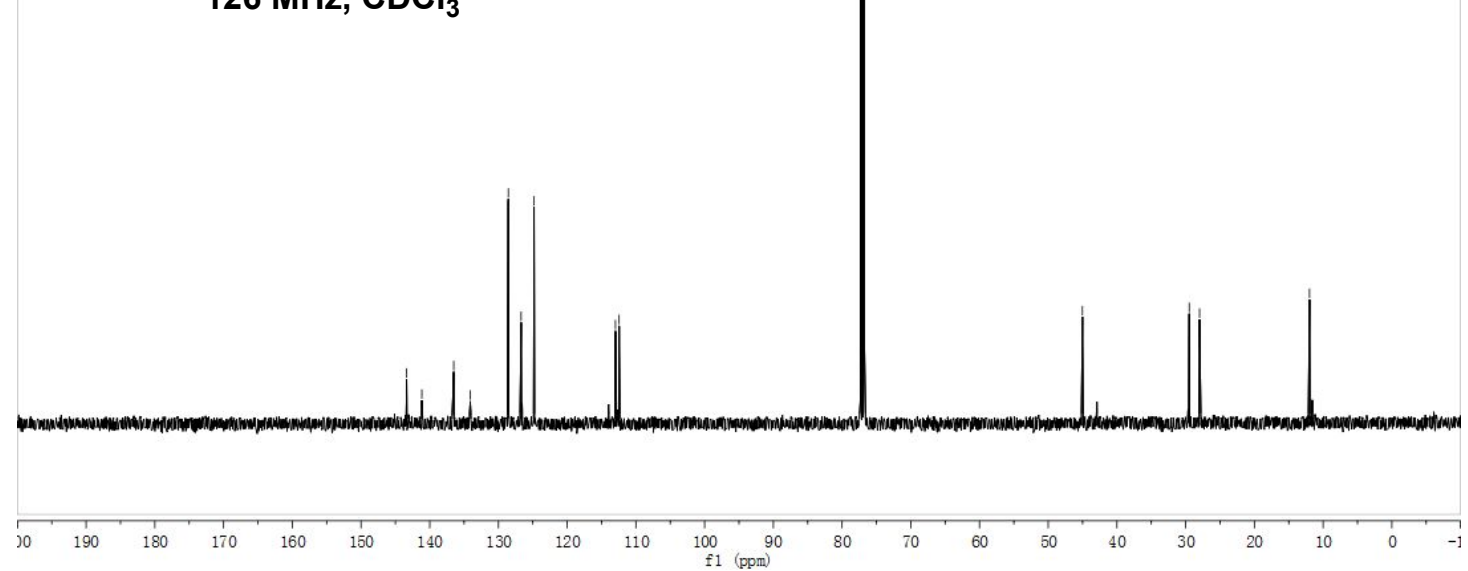


WJW-16 \#12-22 RT: 0.05-0.10 AV: 11 NL: 1.68E9

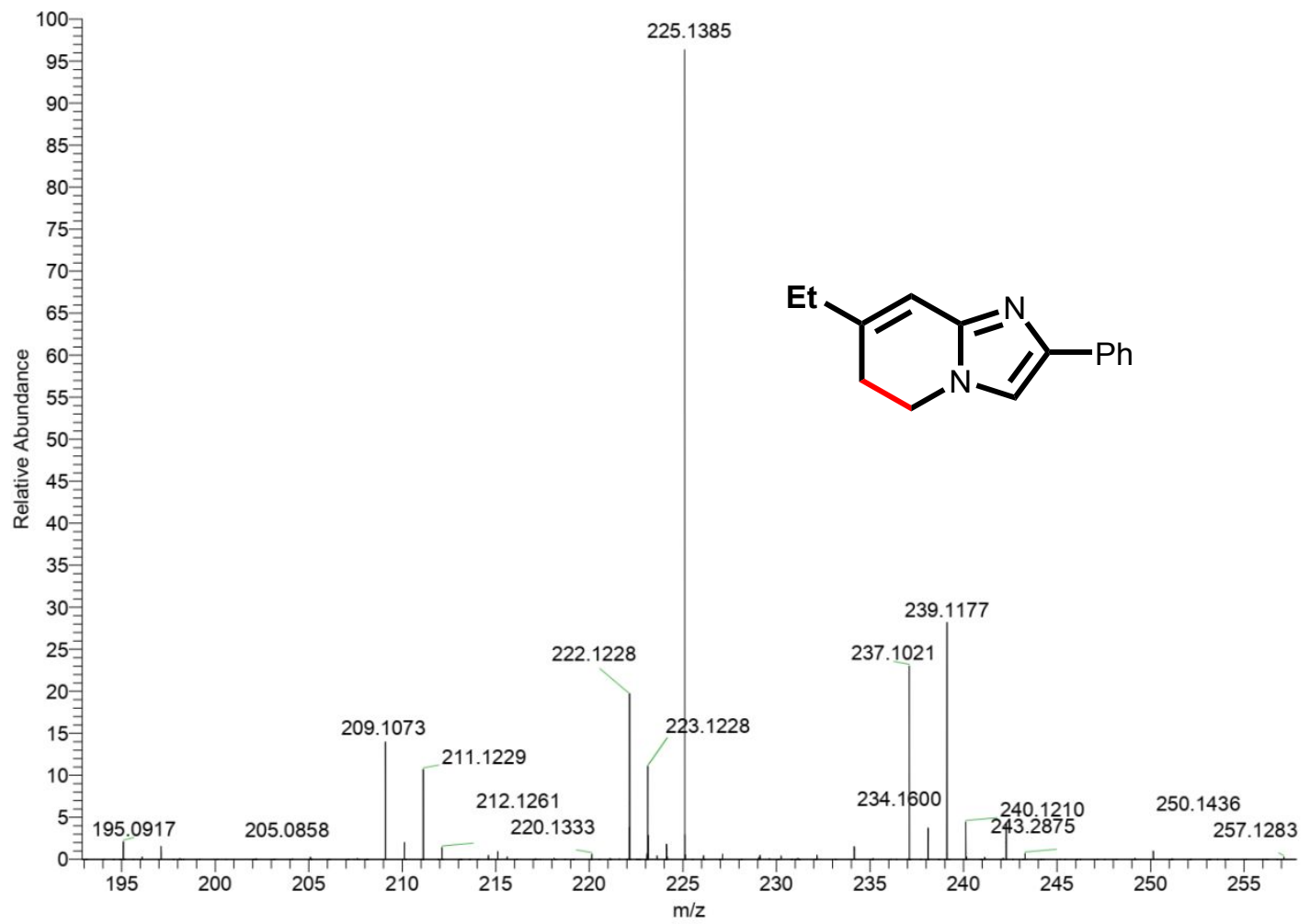


${ }^{1}$ HNMR

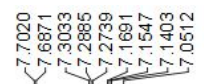

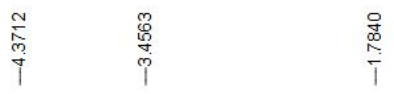

$500 \mathrm{MHz}, \mathrm{CDCl}_{3}$

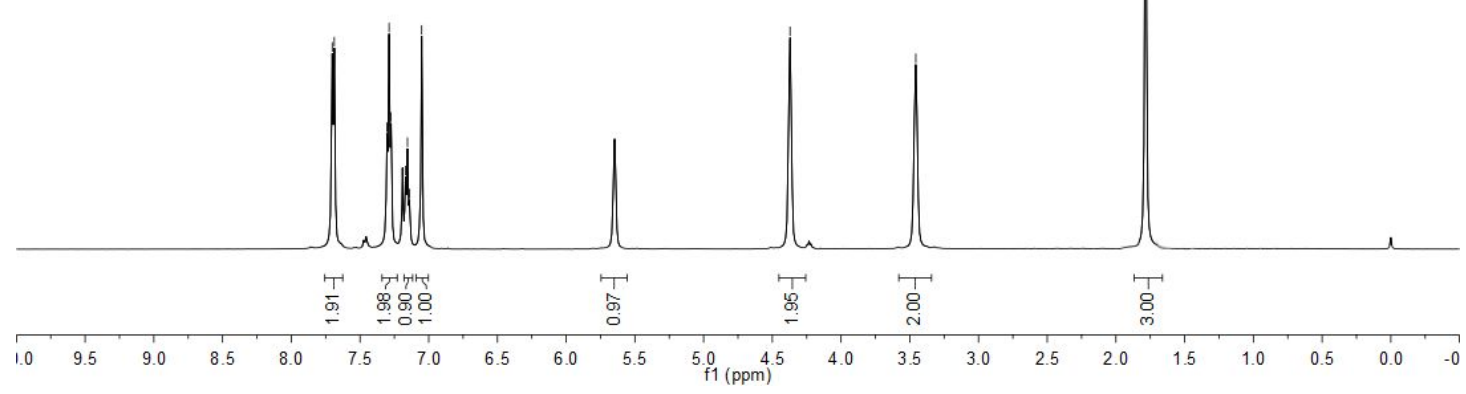

${ }^{13}$ CNMR

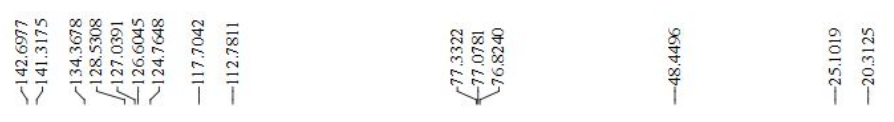

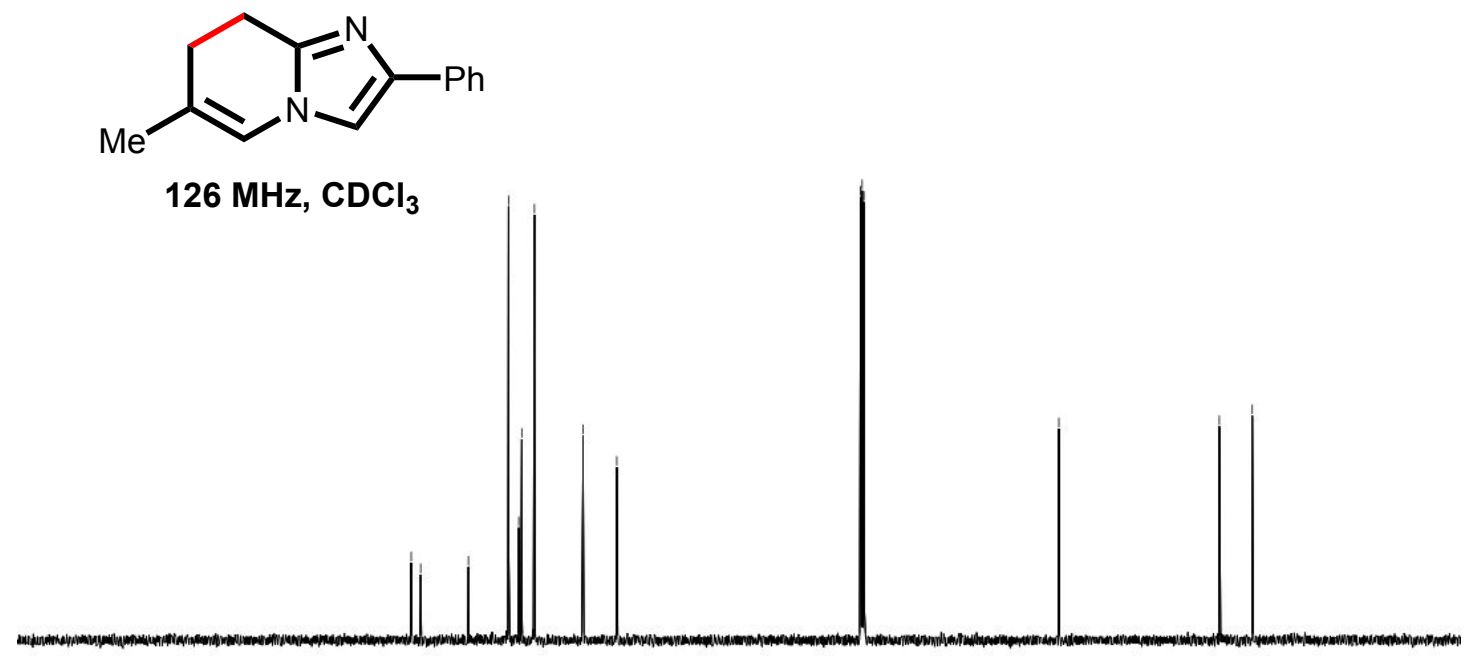

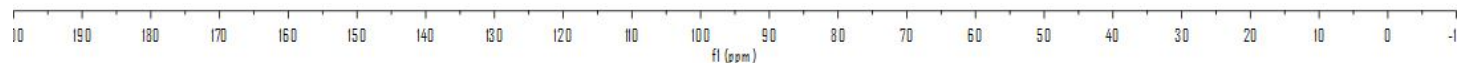


WJW-12 \#13-29 RT: 0.05-0.10 AV: 12 SB: 243 0.77-1.31, 1.40-1.94 NL: 1.02E10 T: FTMS + p ESI Full ms [100.0000-1000.0000]

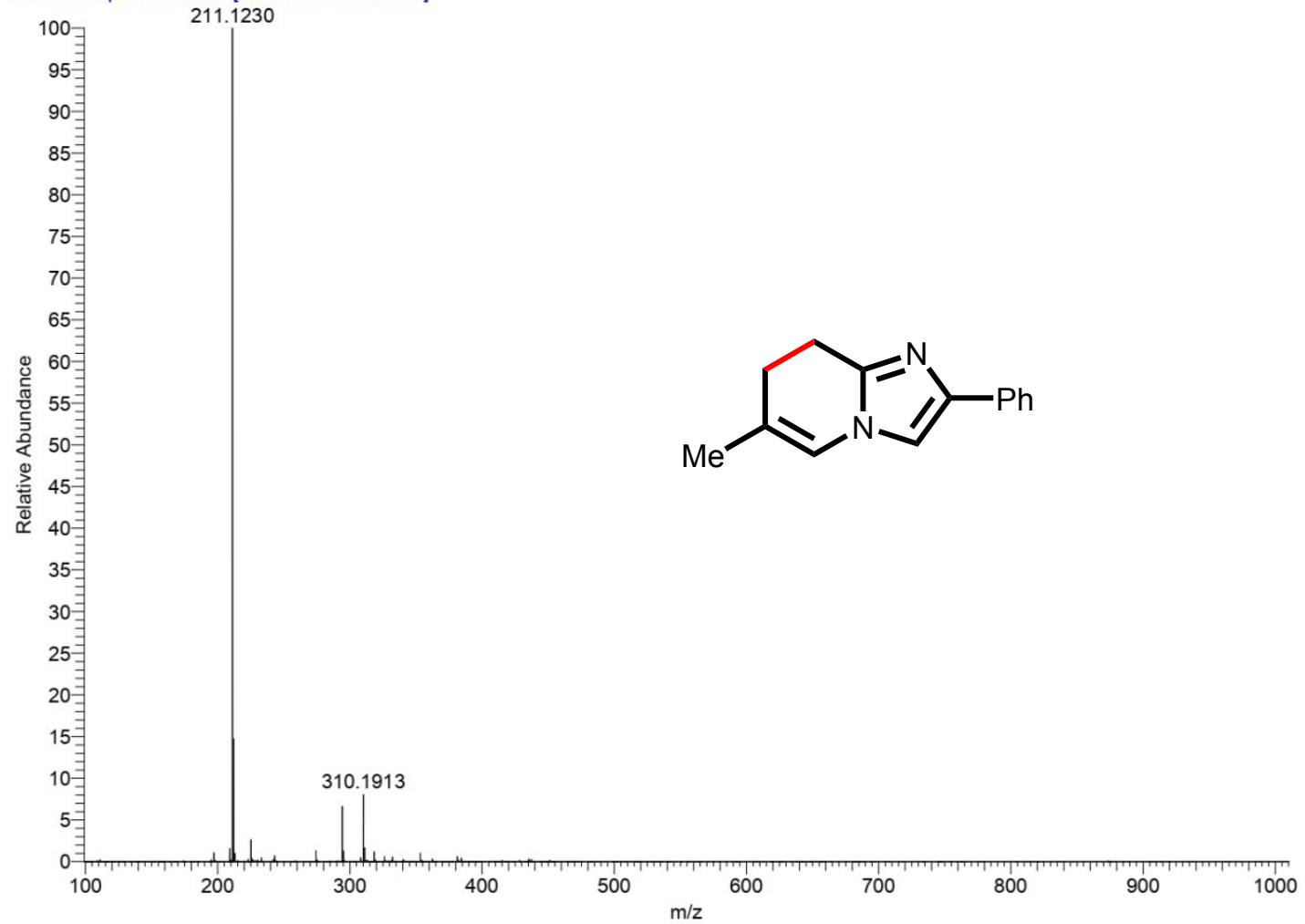


${ }^{1}$ HNMR

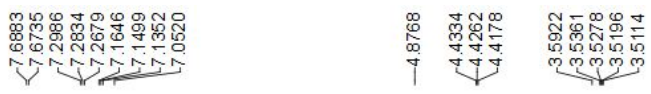

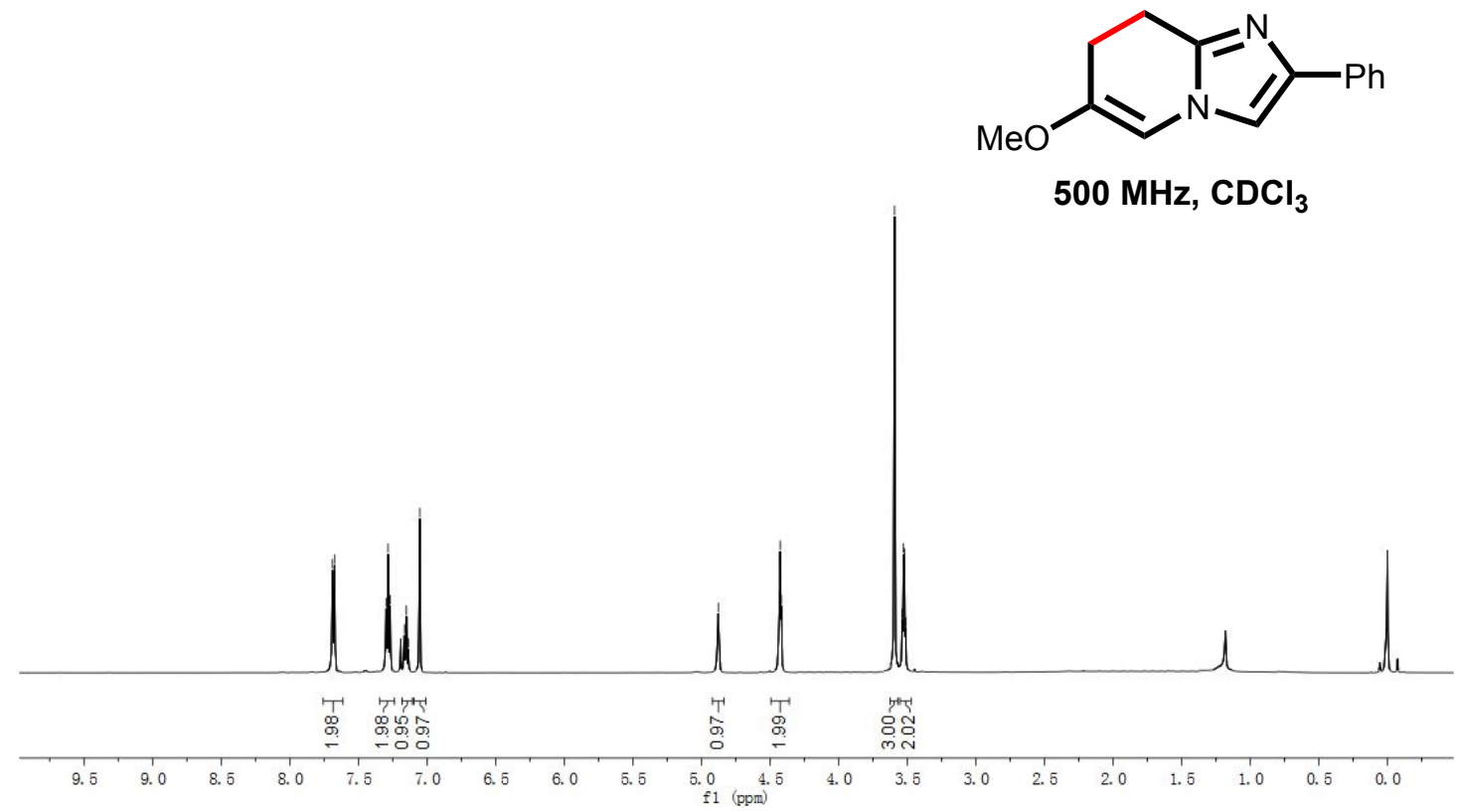

${ }^{13}$ CNMR

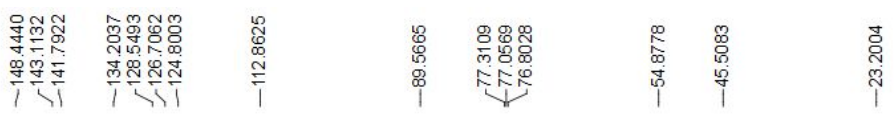

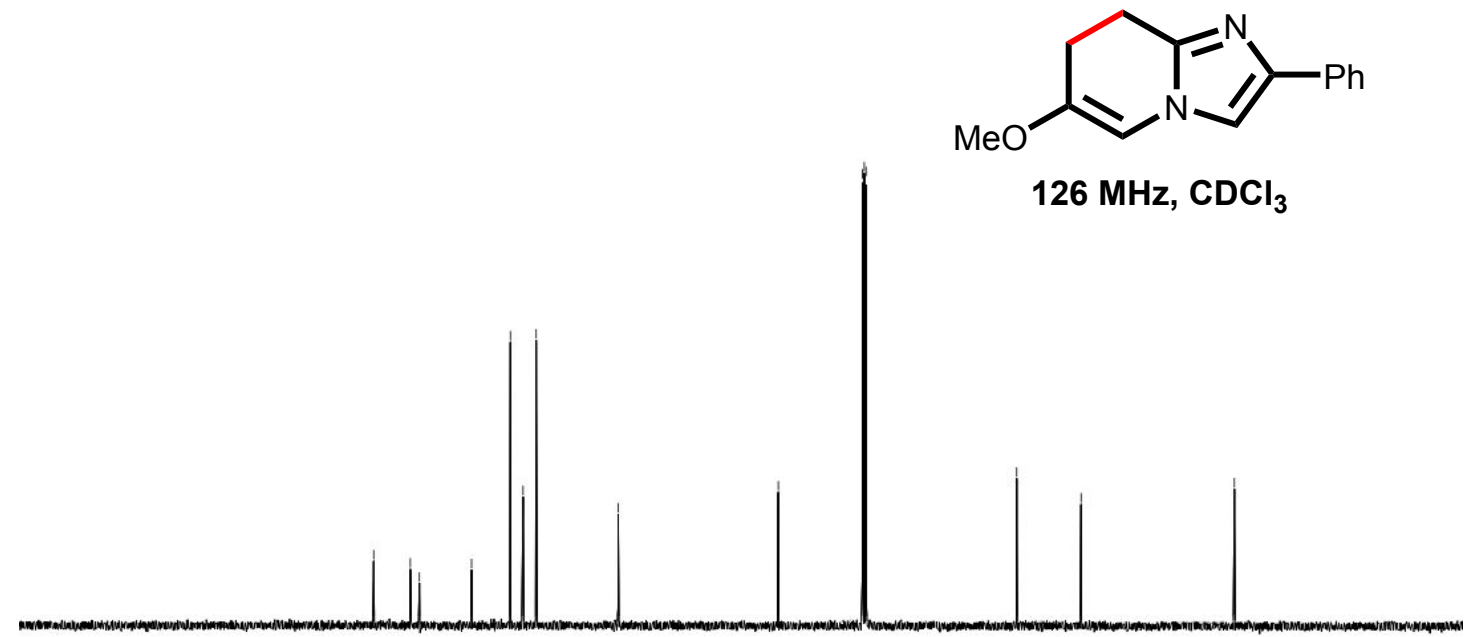

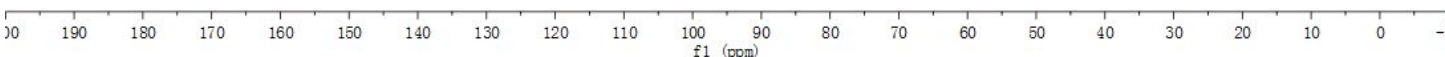


WJW-4 \#15-24 RT: 0.06-0.10 AV: 10 NL: 1.78E10

T: FTMS + p ESI Full ms [100.0000-1000.0000]

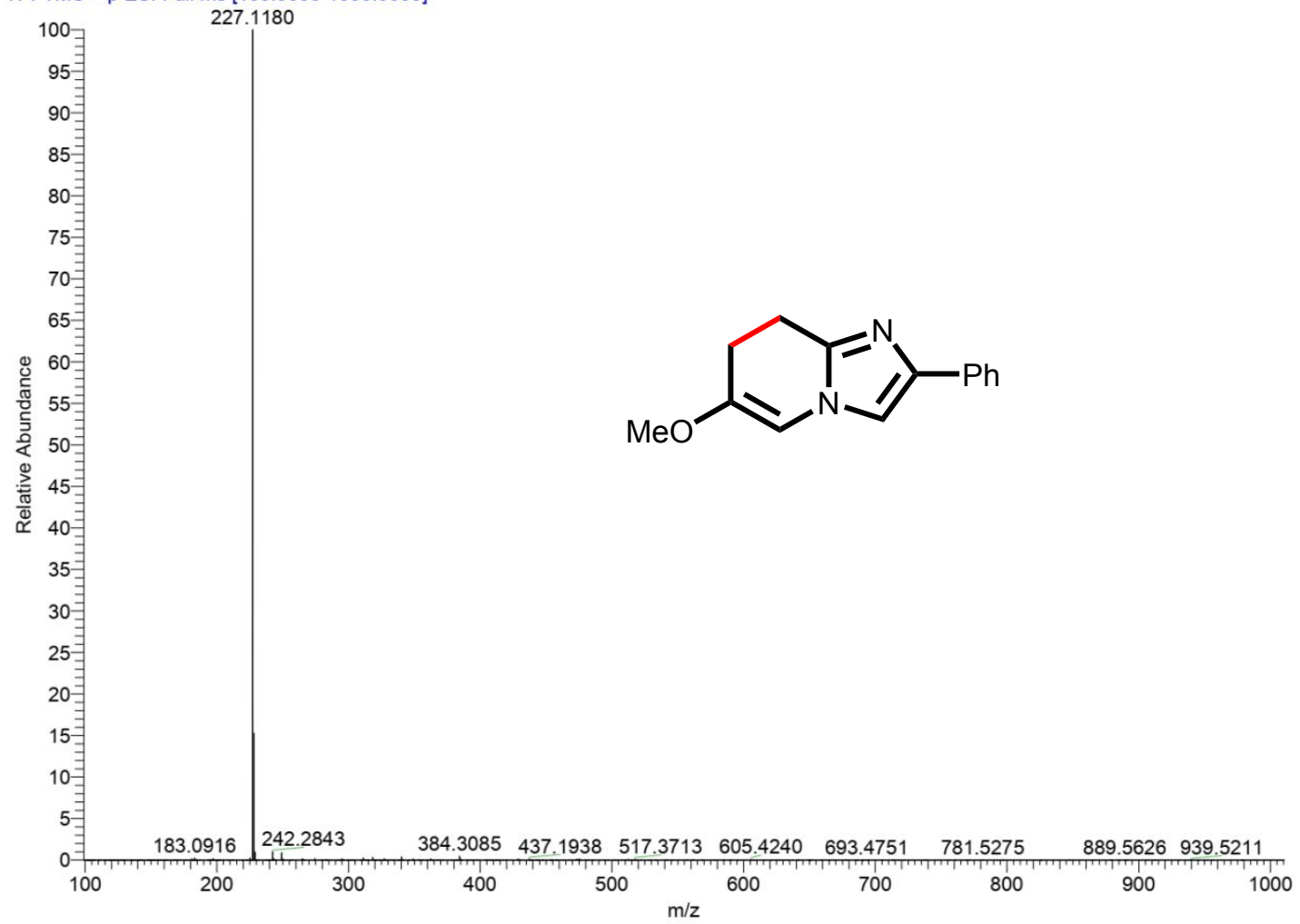




\section{${ }^{1}$ HNMR}

$2 \mathrm{~g}$
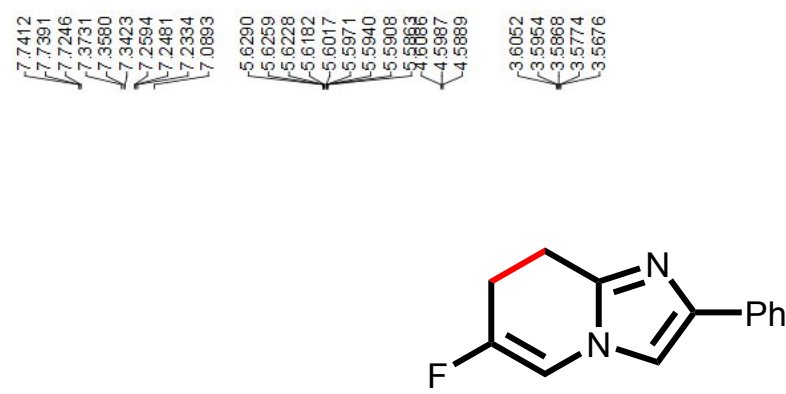

$500 \mathrm{MHz}, \mathrm{CDCl}_{3}$

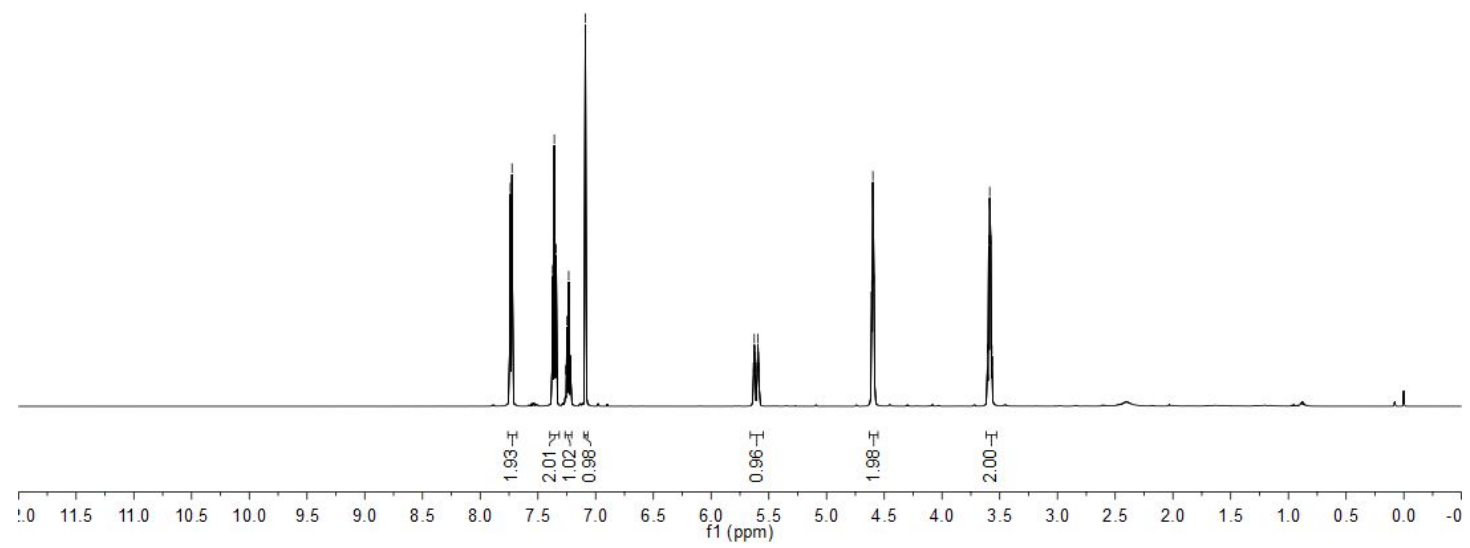

${ }^{13}$ CNMR

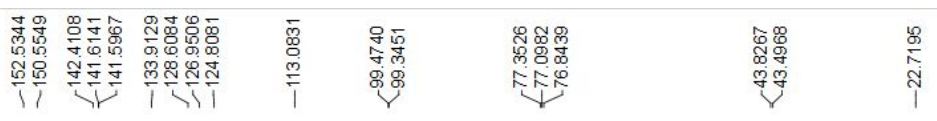<smiles>FC1=Cn2cc(-c3ccccc3)nc2CC1</smiles>

$126 \mathrm{MHz}^{\mathrm{CDCl}_{3}}$

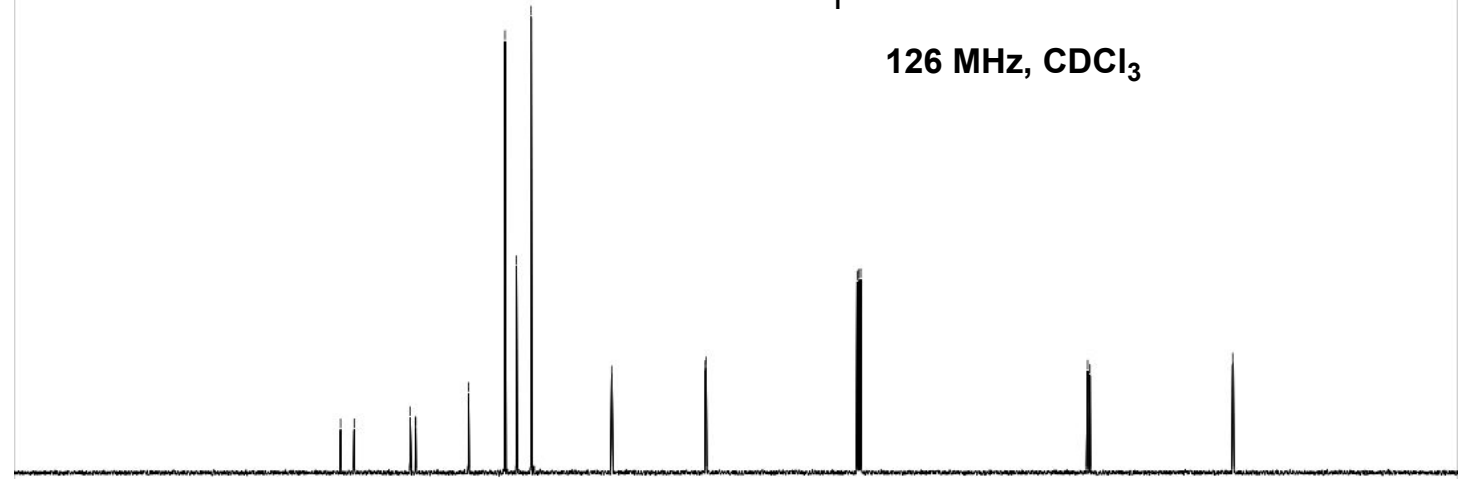

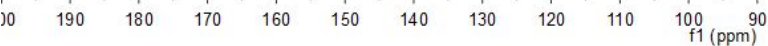




\section{${ }^{19}$ FNMR}

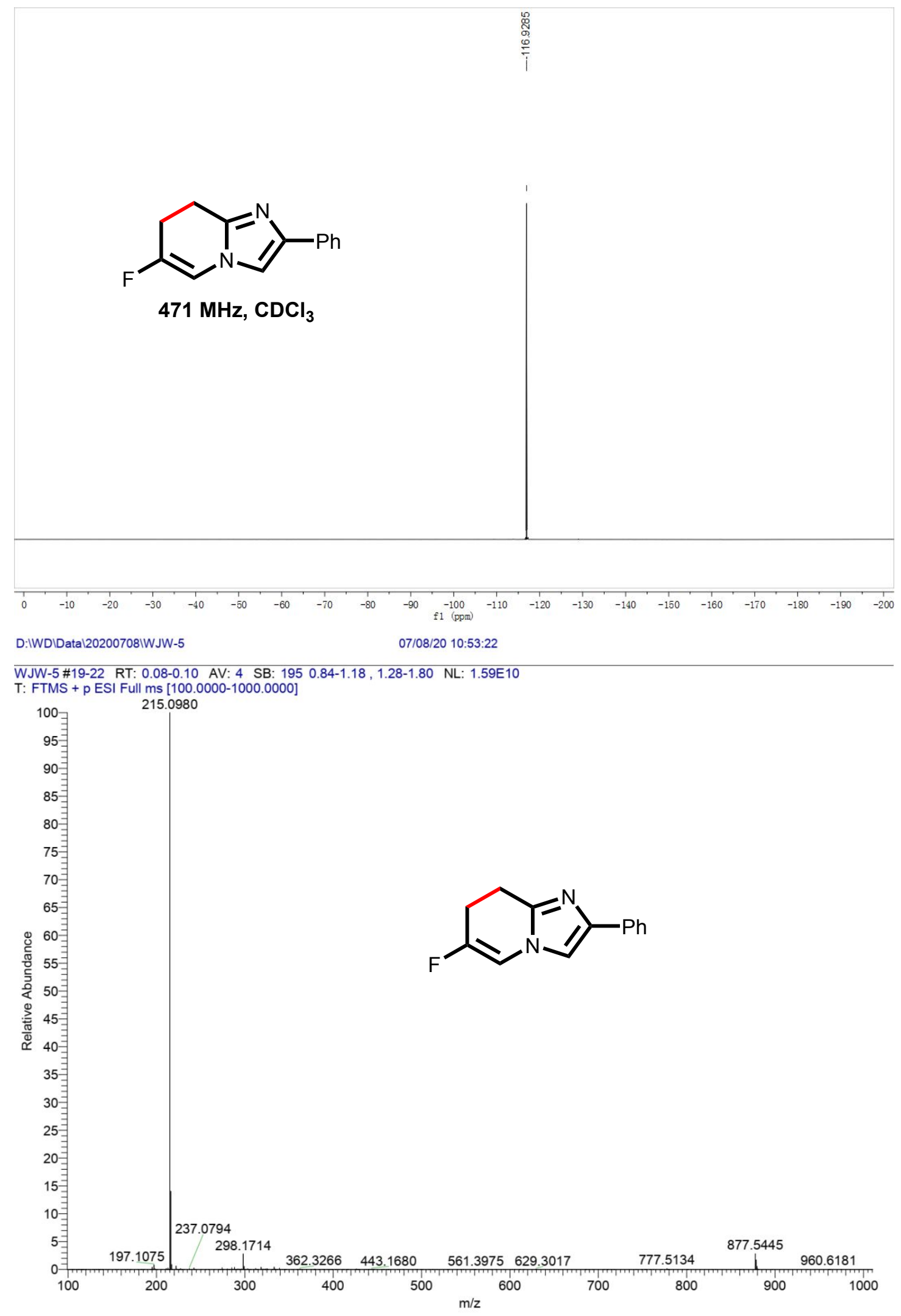


${ }^{1}$ HNMR

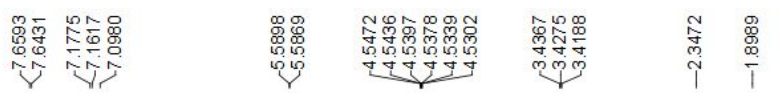<smiles>CC1=Cc2nc(-c3ccc(C)cc3)cn2CC1</smiles>

$500 \mathrm{MHz}^{\mathrm{CDCl}_{3}}$

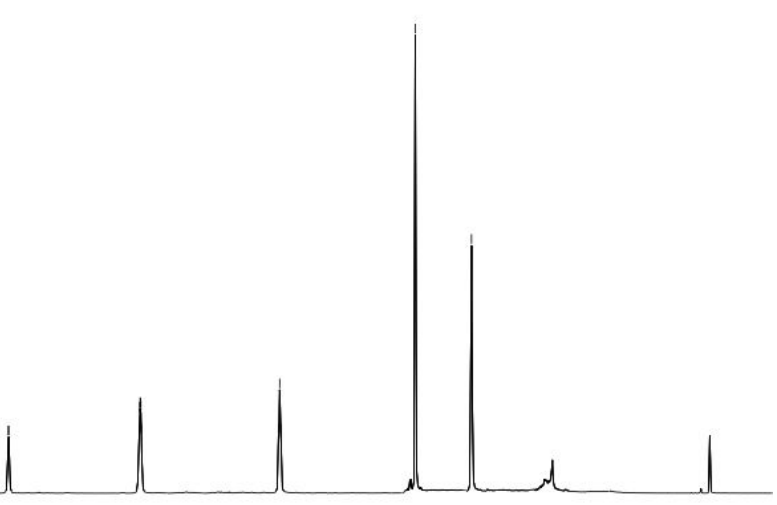

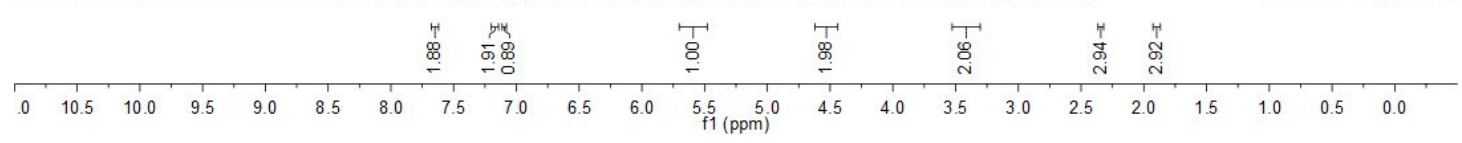

${ }^{13}$ CNMR

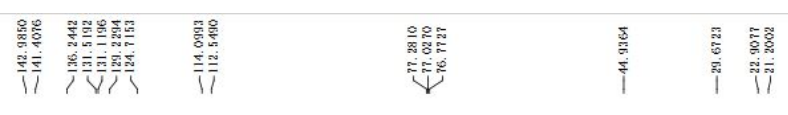<smiles>CC1=Cc2nc(-c3ccc(C)cc3)cn2CC1</smiles>

$126 \mathrm{MHz}^{\mathrm{CDCl}_{3}}$

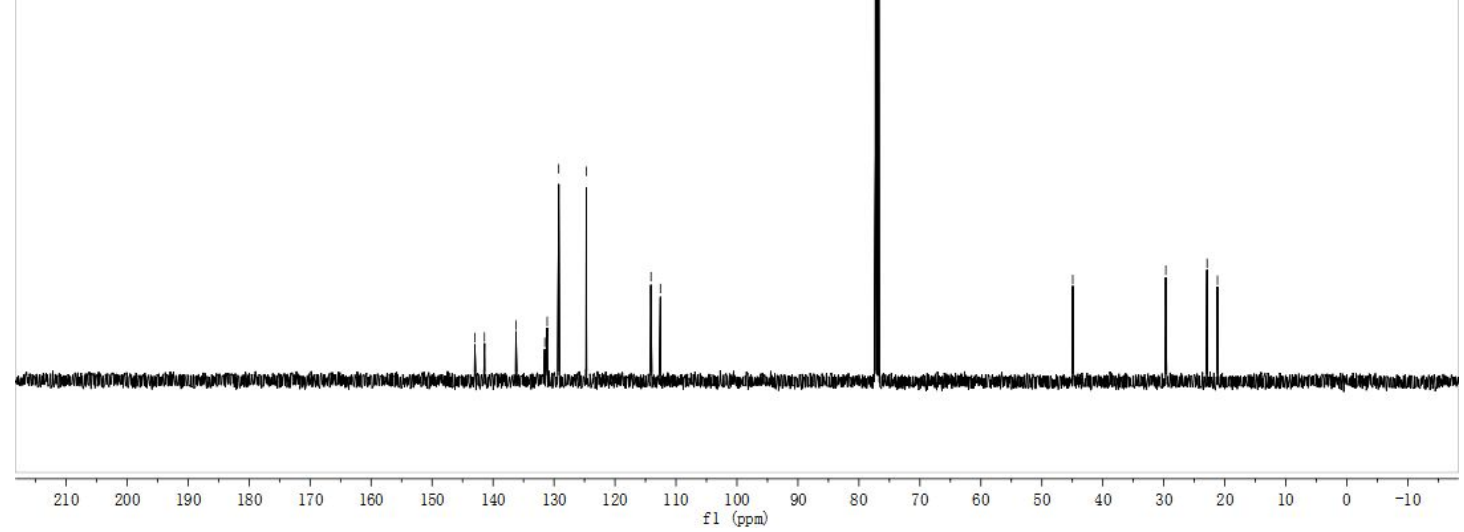


WJW-6 \#22-28 RT: 0.10-0.12 AV: 7 SB: 80 0.85-1.20 NL: 8.60E9

T: FTMS + p ESI Full ms [100.0000-1000.0000]

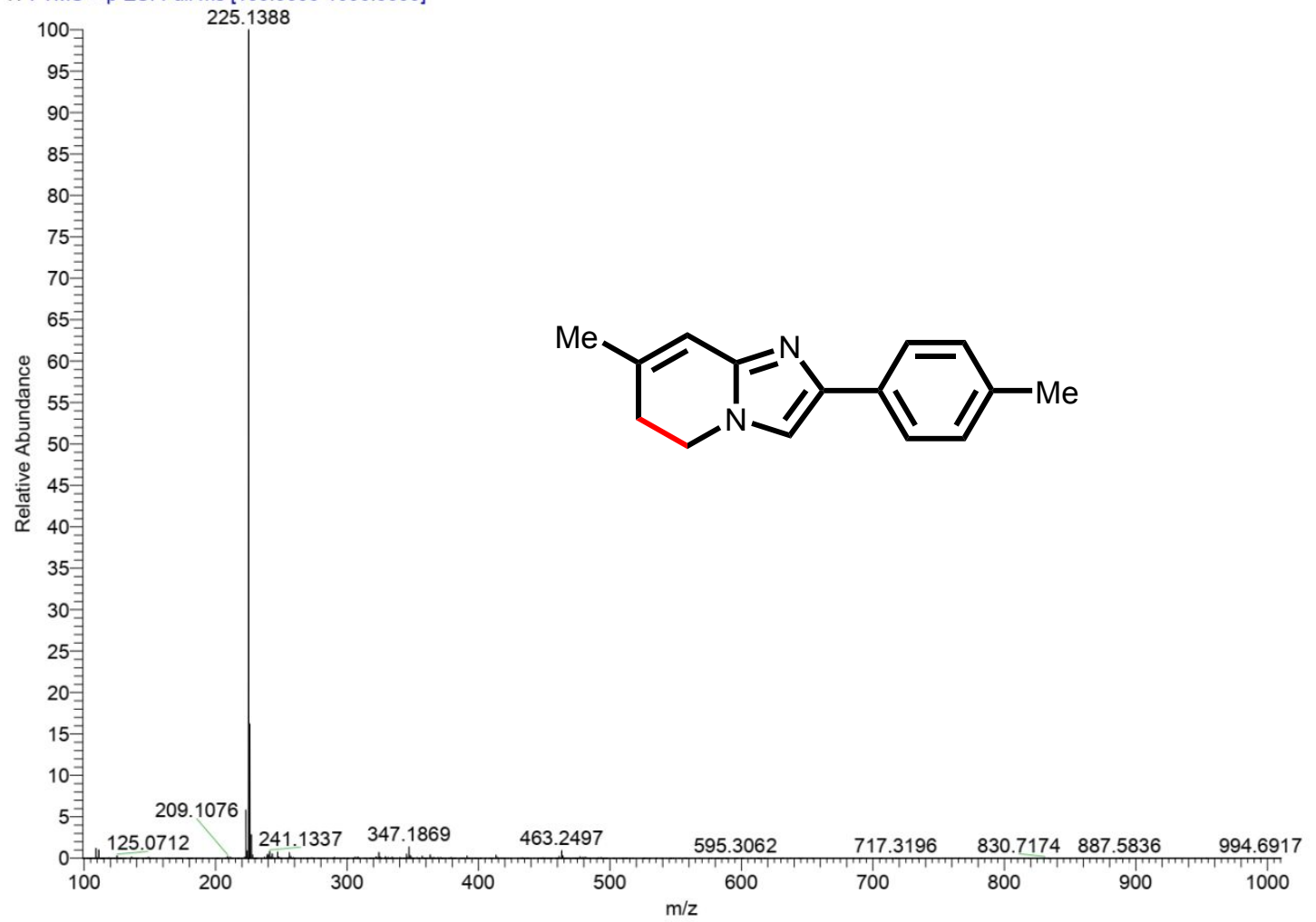


${ }^{1}$ HNMR

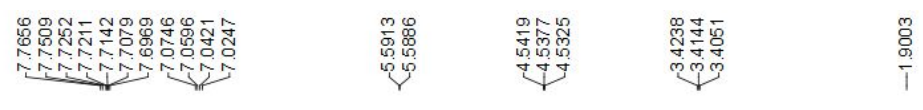<smiles>CC1=Cc2nc(-c3ccc(F)cc3)cn2CC1</smiles>

$500 \mathrm{MHz}^{\mathrm{CDCl}_{3}}$

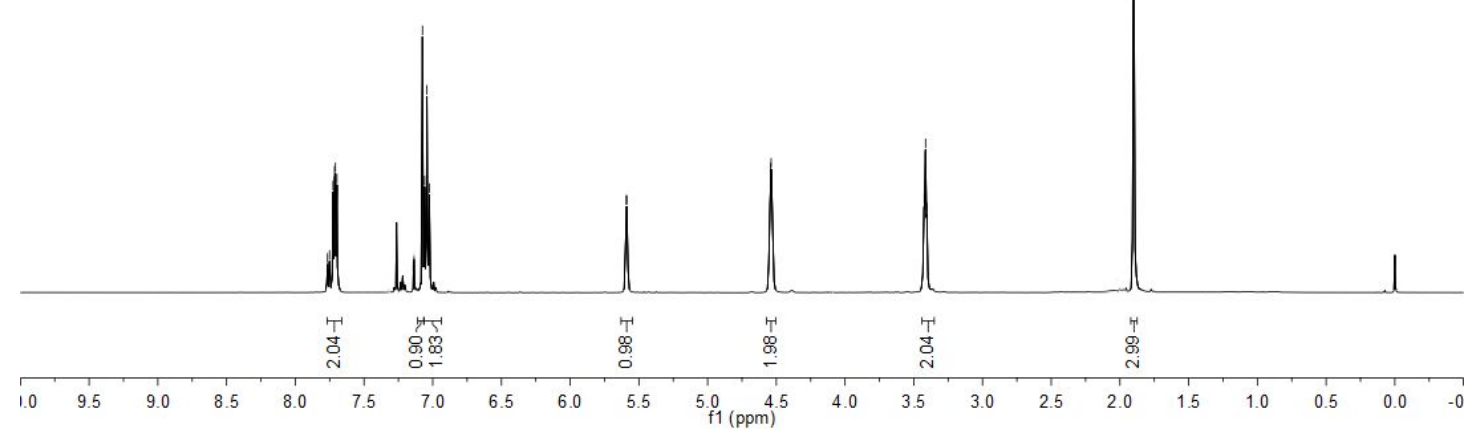

${ }^{13}$ CNMR

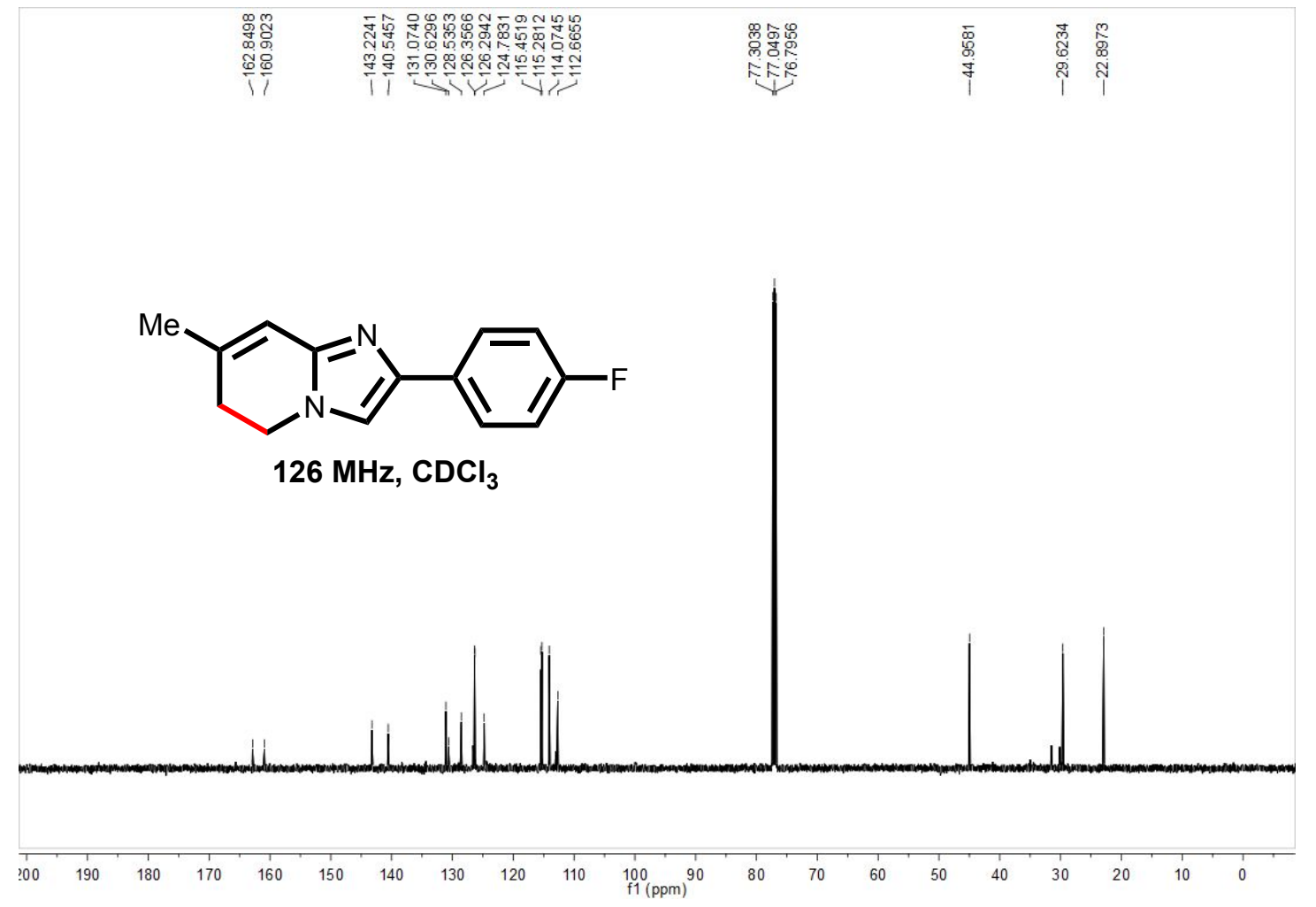


${ }^{19}$ FNMR

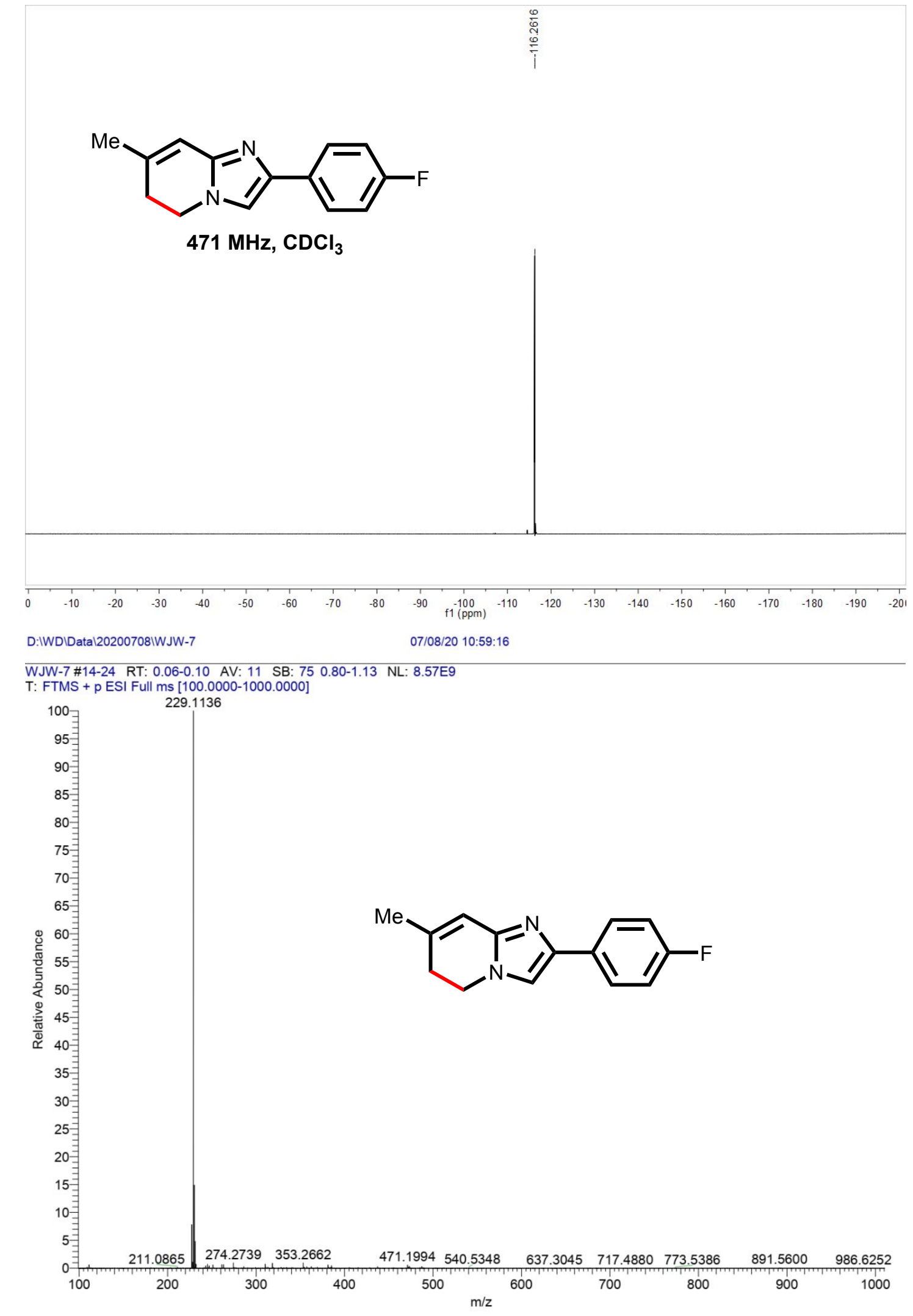


${ }^{1}$ HNMR

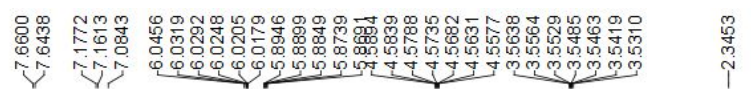<smiles>Cc1ccc(-c2cn3c(n2)C=CCC3)cc1</smiles>

$500 \mathrm{MHz}, \mathrm{CDCl}_{3}$

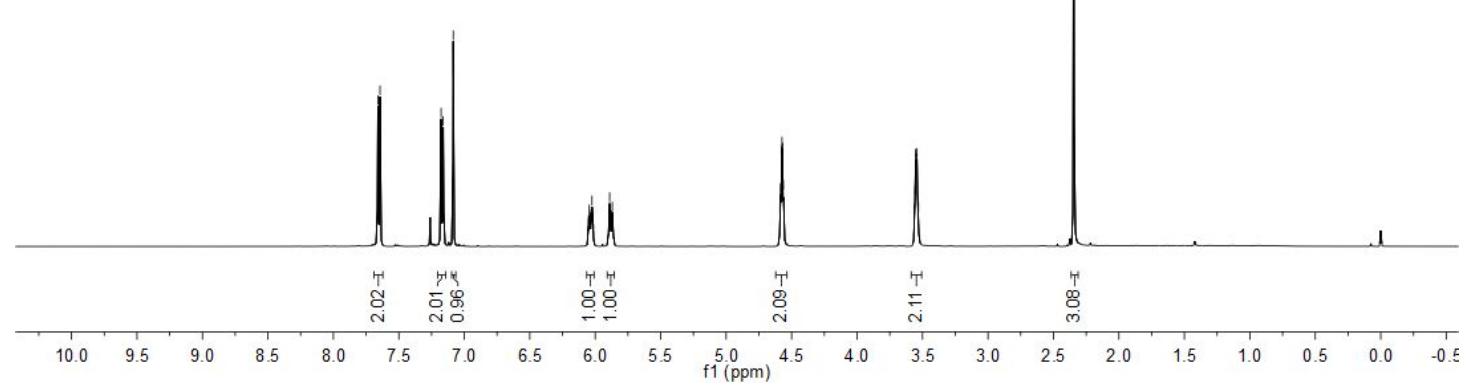

${ }^{13}$ CNMR

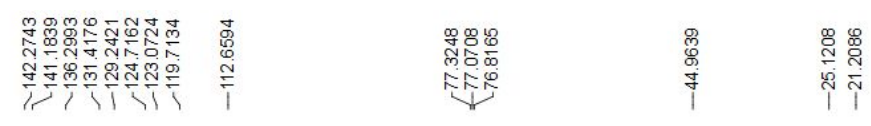

$C_{N}^{N}=M$

$126 \mathrm{MHz}, \mathrm{CDCl}_{3}$

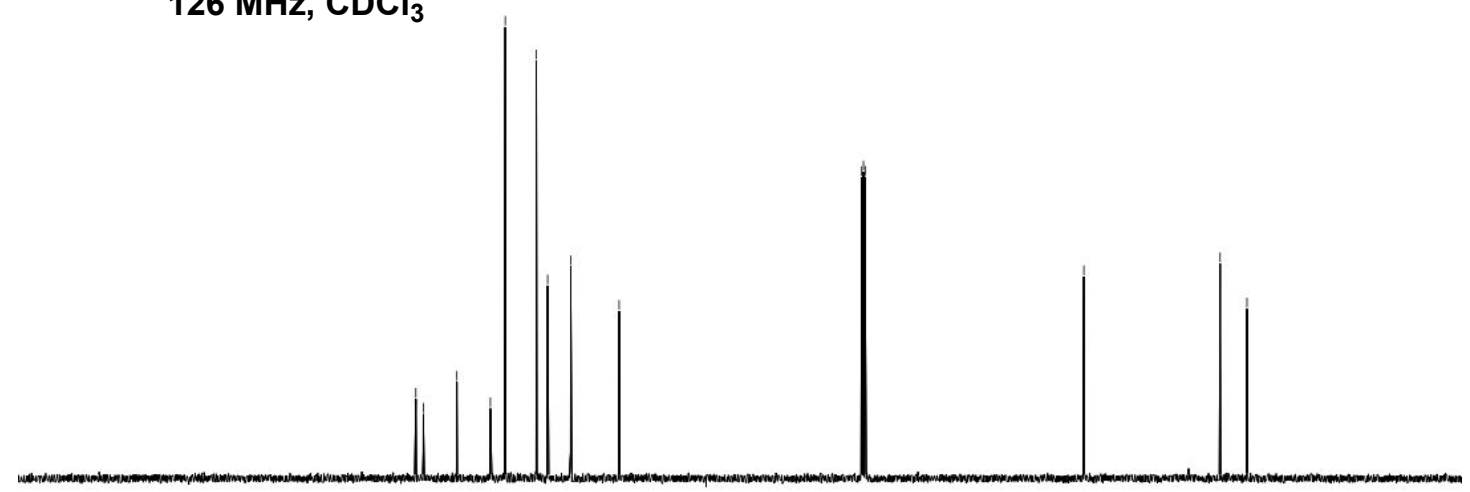

$\begin{array}{llllllllllllllllllllllllllllllllll}10 & 190 & 180 & 170 & 160 & 150 & 140 & 130 & 120 & 110 & 100 & 90 & 80 & 70 & 60 & 50 & 40 & 30 & 20 & 10 & 0 & -1\end{array}$ 
WJW-13 \#13-24 RT: 0.05-0.10 AV: 12 SB: 243 0.77-1.31, 1.40-1.94 NL: 1.02E10 T: FTMS + p ESI Full ms [100.0000-1000.0000]

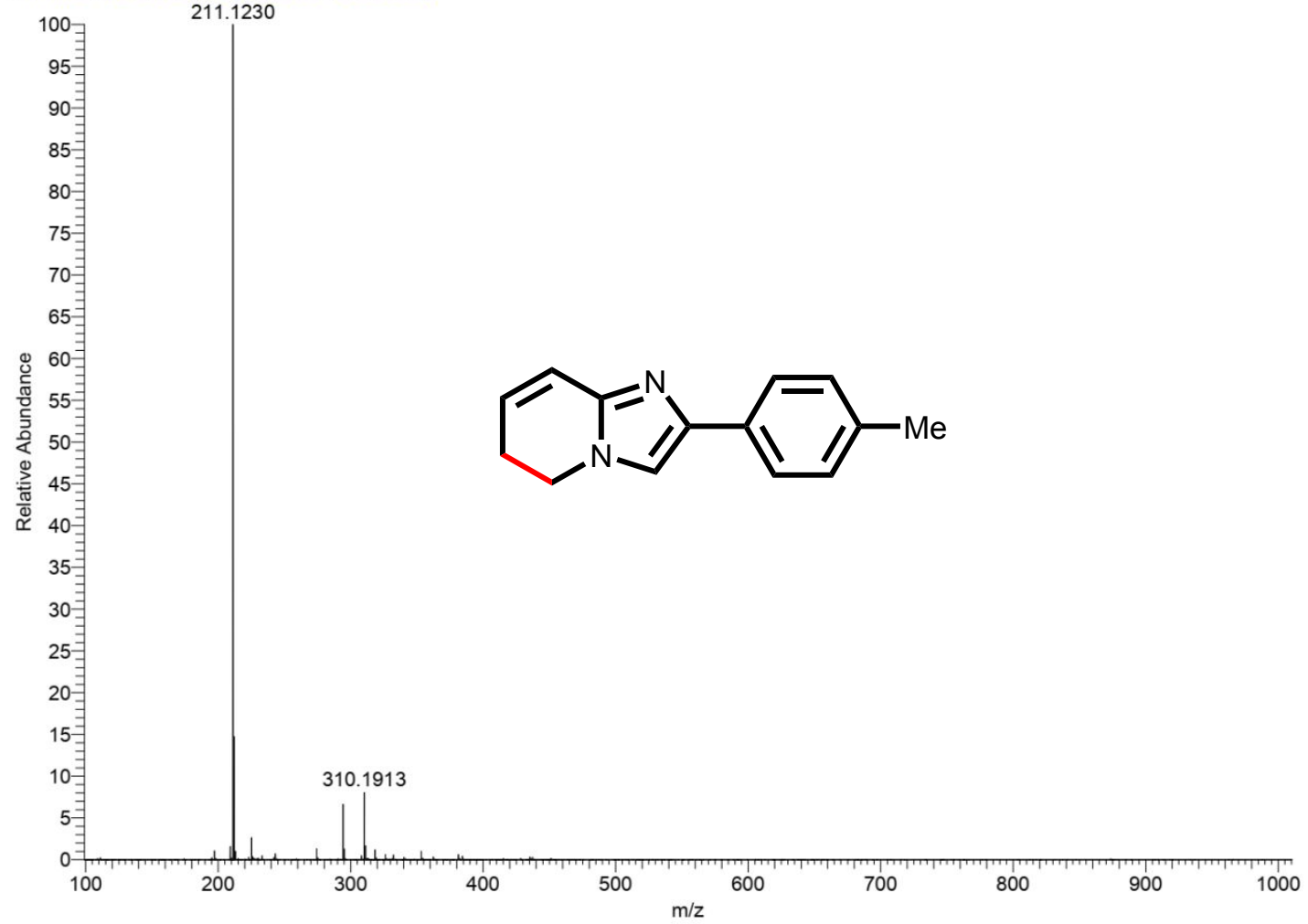


1HNMR

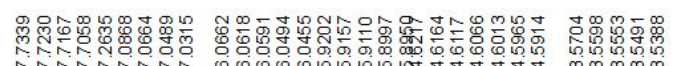

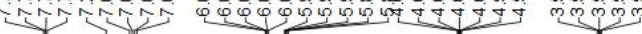

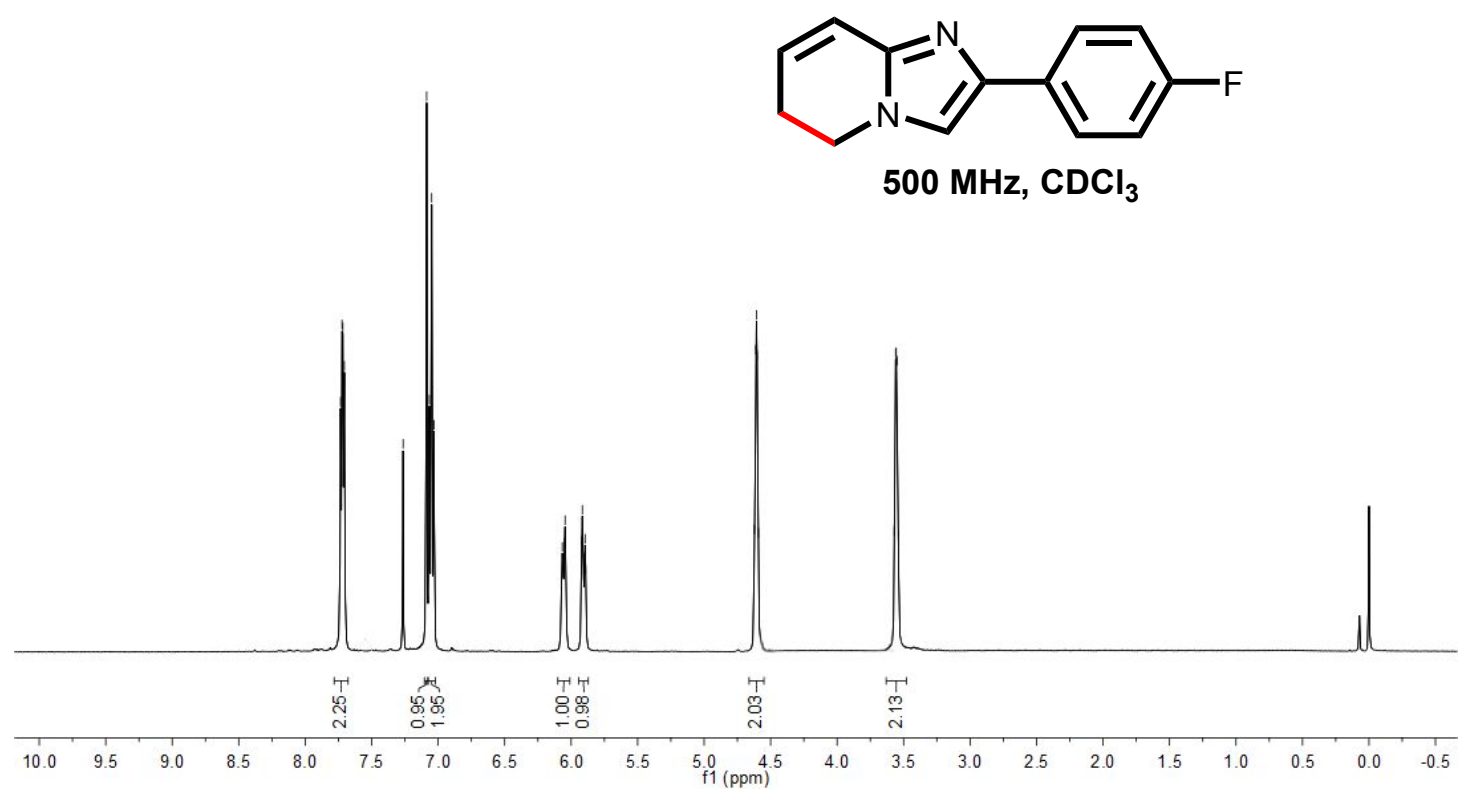

${ }^{13}$ CNMR

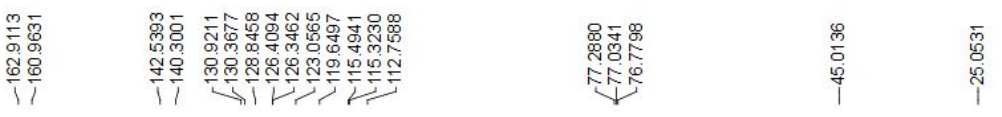<smiles>Fc1ccc(-c2cn3c(n2)C=CCC3)cc1</smiles>

$126 \mathrm{MHz}^{\mathrm{CDCl}_{3}}$

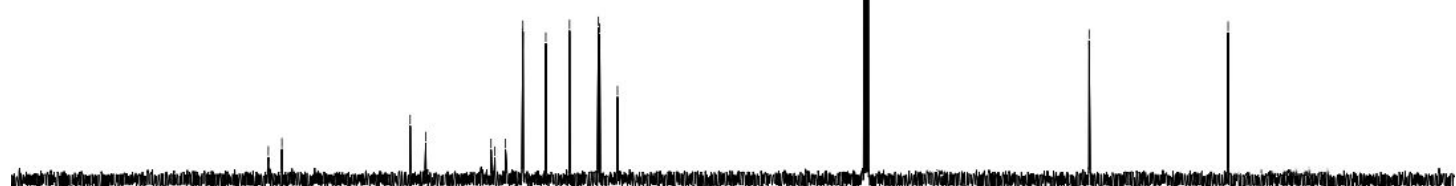

$\begin{array}{llllllllll}190 & 180 & 170 & 160 & 150 & 140 & 130 & 120 & 110 & \underset{f 1}{100}(\mathrm{ppm})\end{array}$ 
${ }^{19}$ FNMR

$r_{N}^{N}-{ }_{-F}$

$471 \mathrm{MHz}^{\mathrm{CDCl}_{3}}$

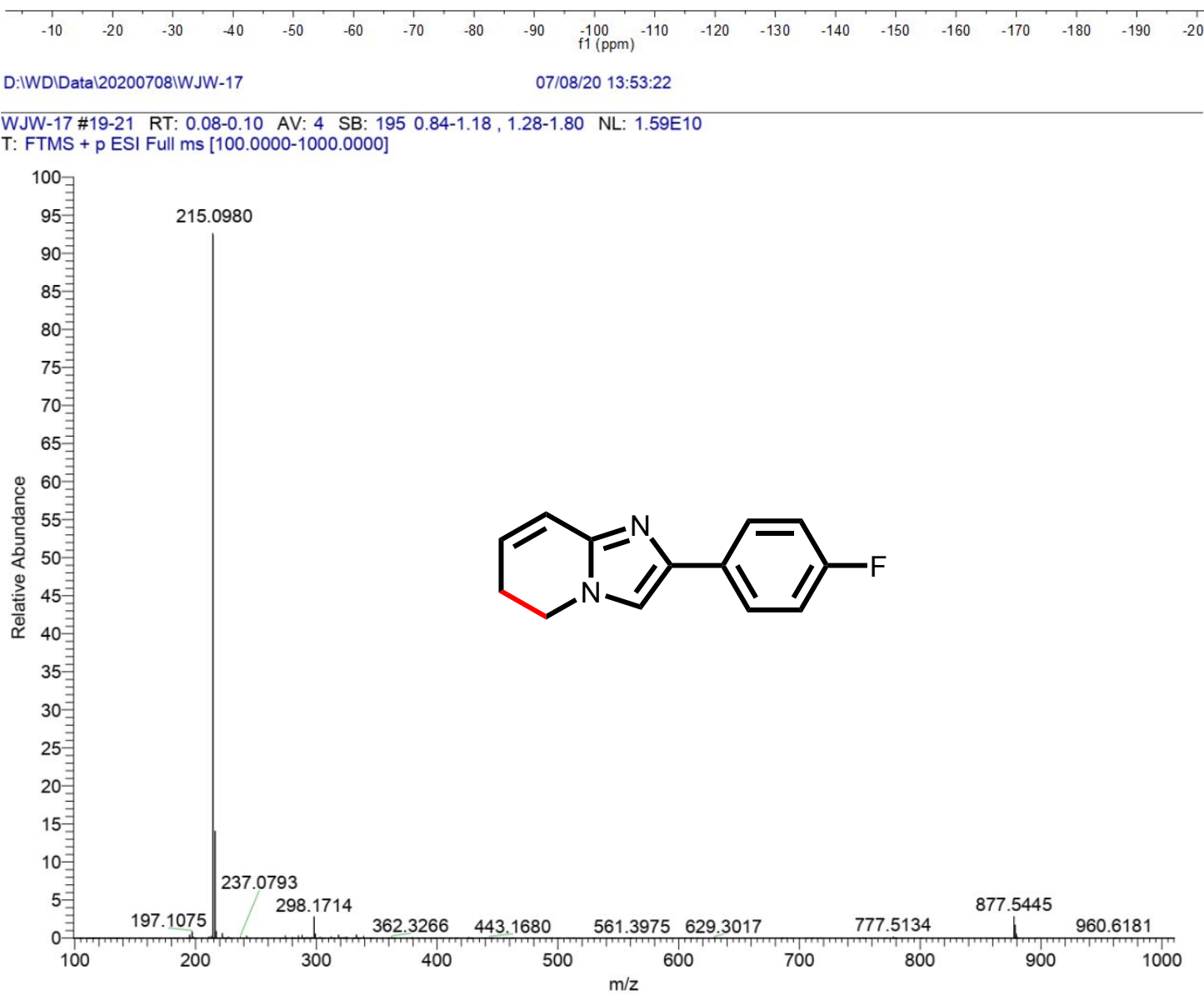


${ }^{1}$ HNMR

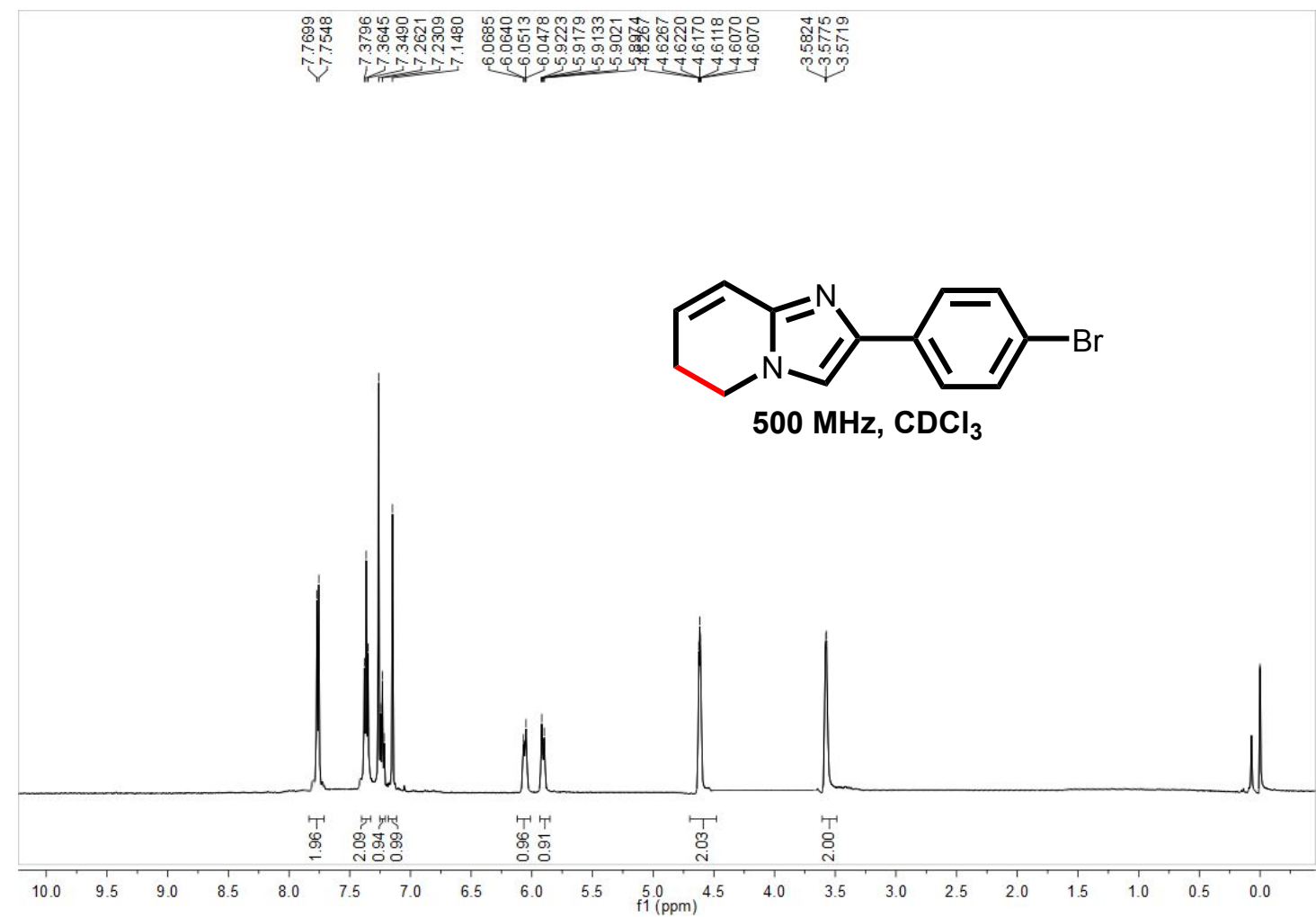

${ }^{13}$ CNMR

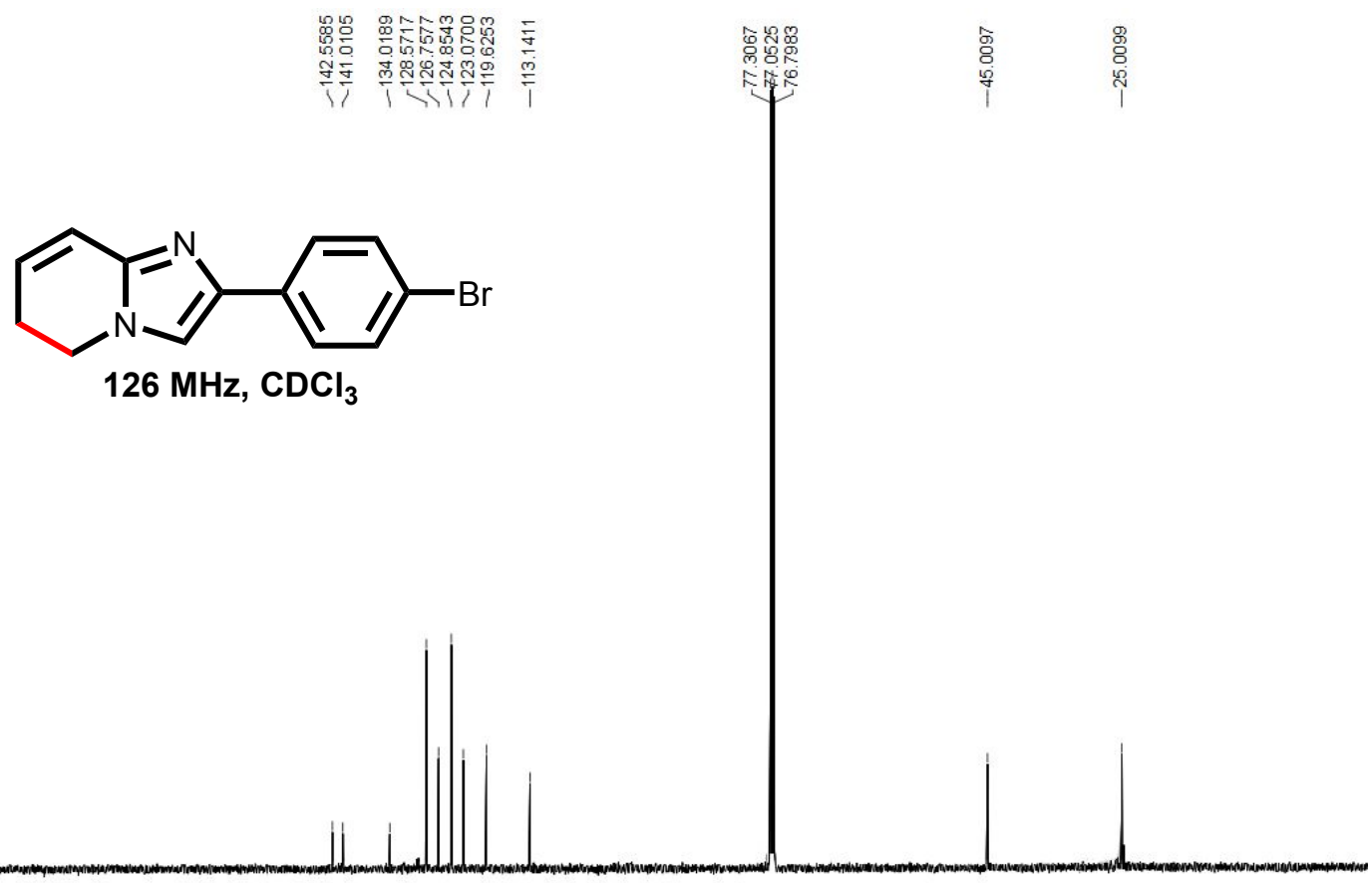

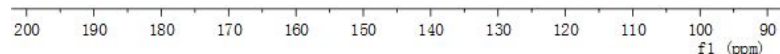




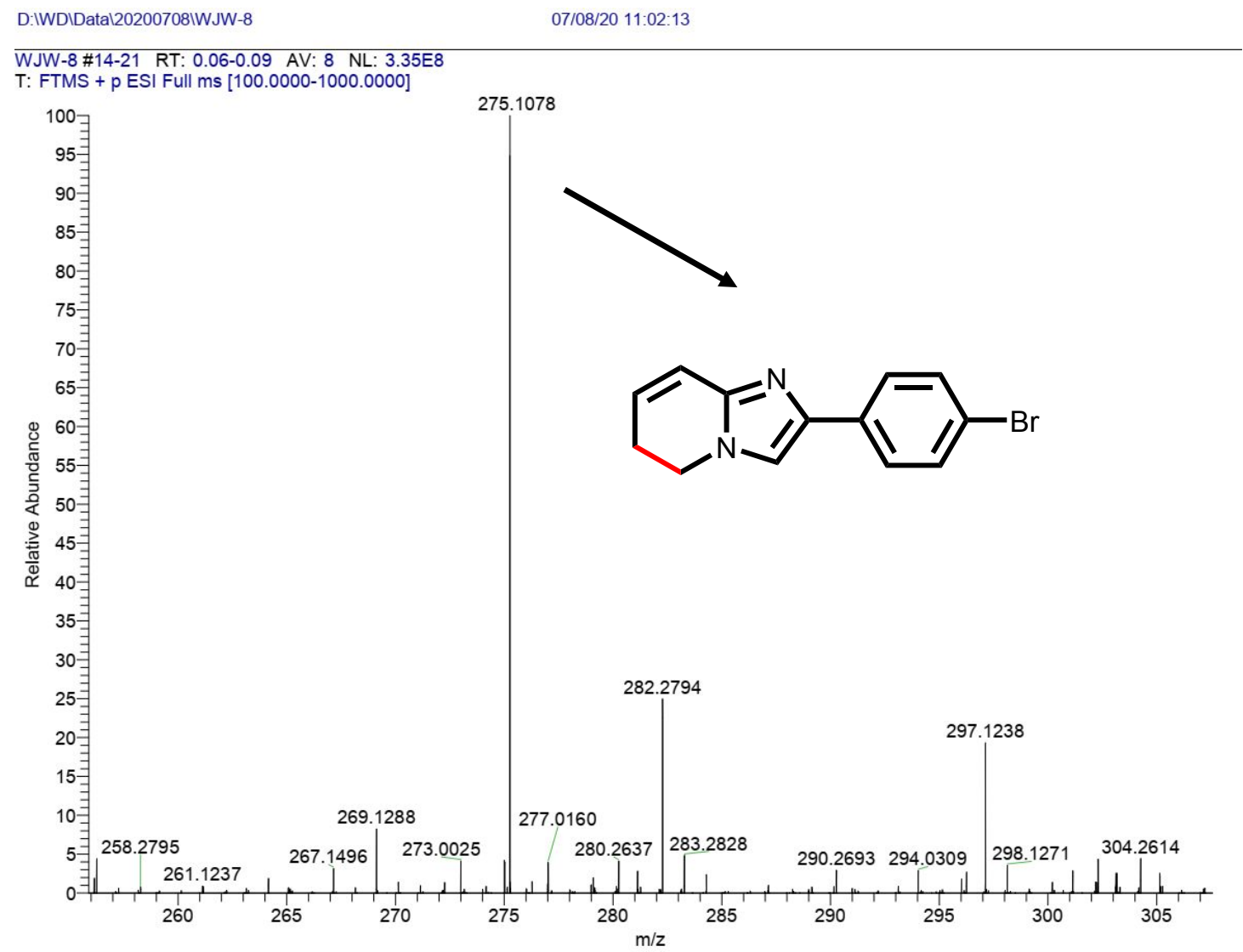


${ }^{1}$ HNMR

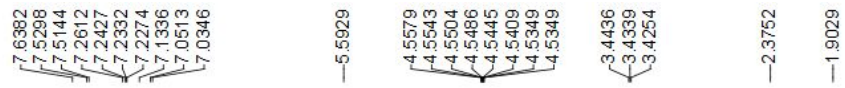<smiles>CC1=Cc2nc(-c3cccc(C)c3)cn2CC1</smiles>

$500 \mathrm{MHz} \mathrm{CDCl}_{3}$

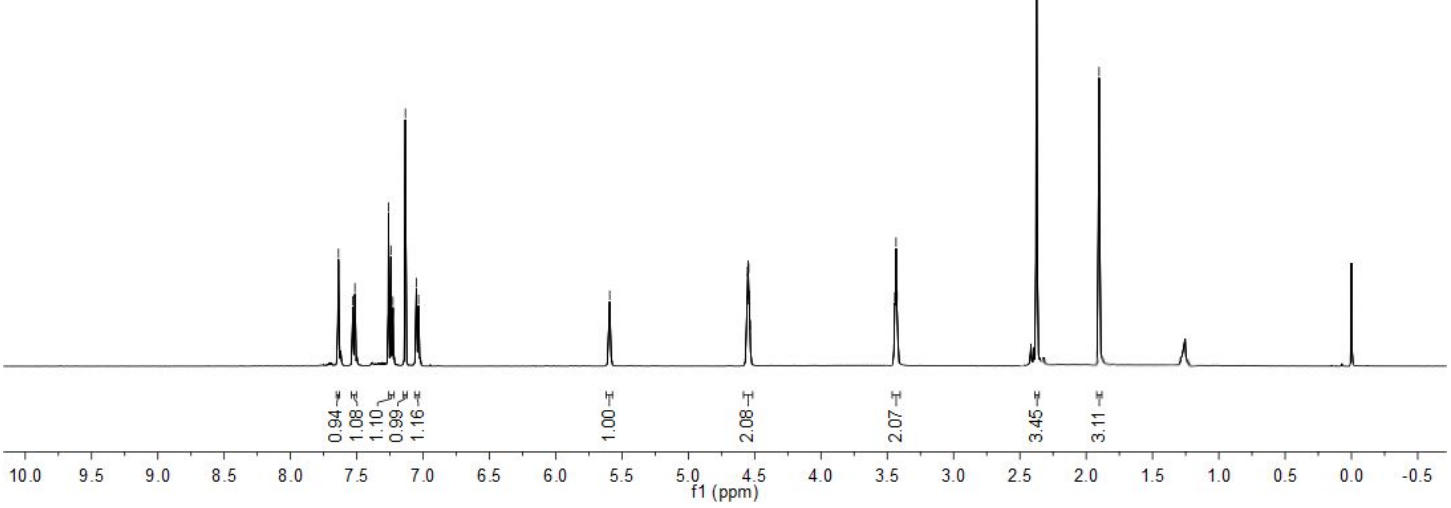

${ }^{13}$ CNMR
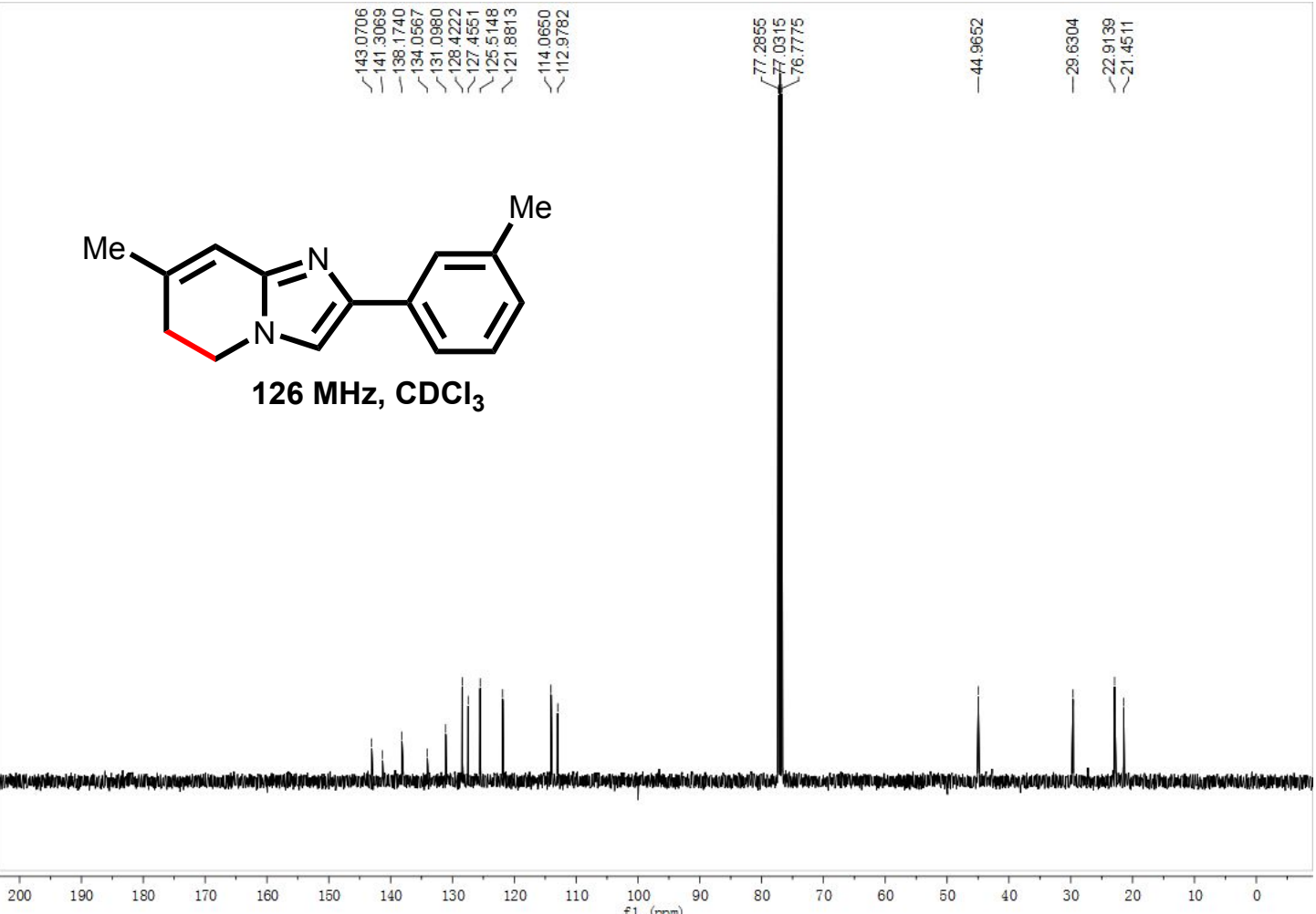
WJW-15\#22-20 RT: 0.10-0.12 AV: 7 SB: 80 0.85-1.20 NL: 8.60E9

T: FTMS + p ESI Full ms [100.0000-1000.0000]

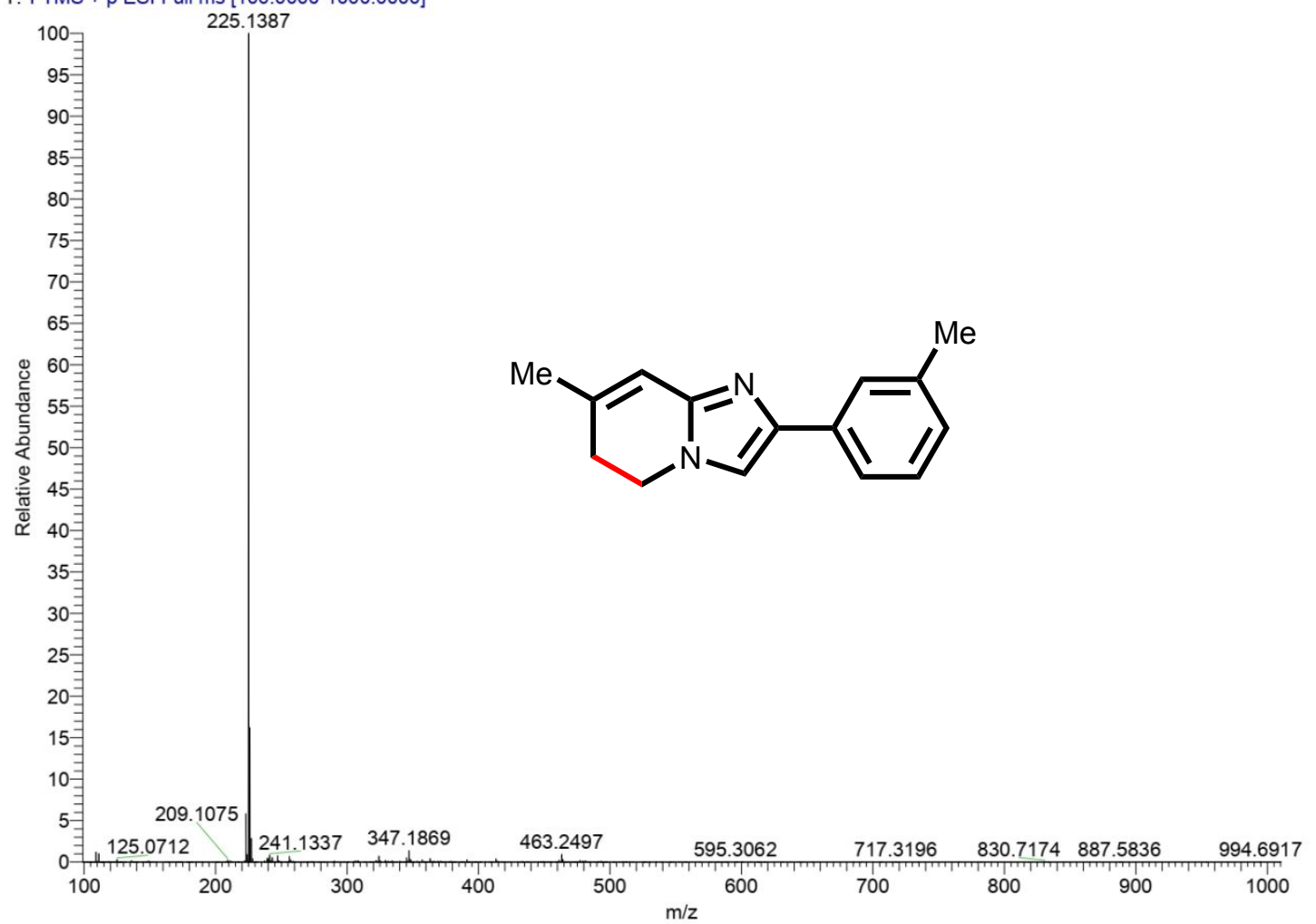


${ }^{1}$ HNMR
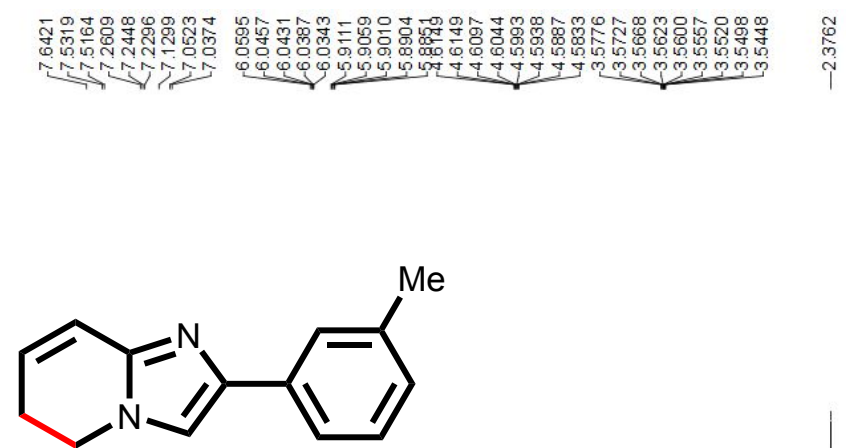

$500 \mathrm{MHz}^{\mathrm{CDCl}_{3}}$

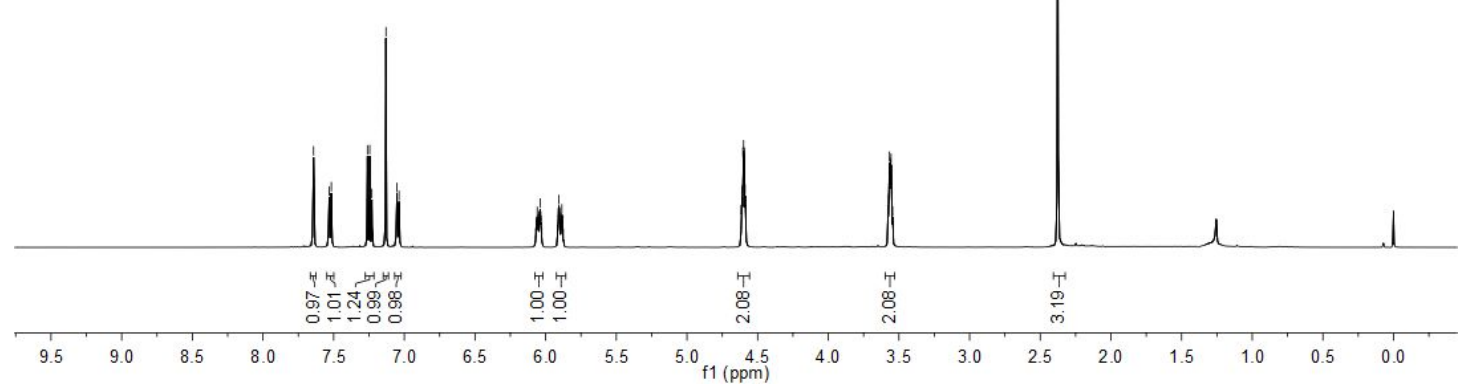

${ }^{13}$ CNMR

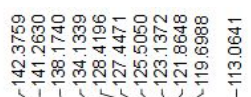

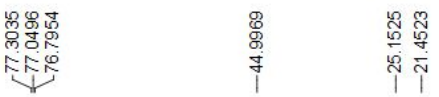

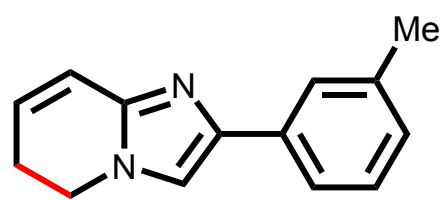

$126 \mathrm{MHz}^{\mathrm{CDCl}_{3}}$

190

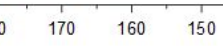

$140 \quad 130$

${ }_{\mathrm{f} 1}^{100}(\mathrm{ppm}){ }^{90}$ 
WJW-21 \#13-25 RT: 0.05-0.10 AV: 12 SB: 243 0.77-1.31, 1.40-1.94 NL: 1.02E10 T: FTMS + p ESI Full ms [100.0000-1000.0000]

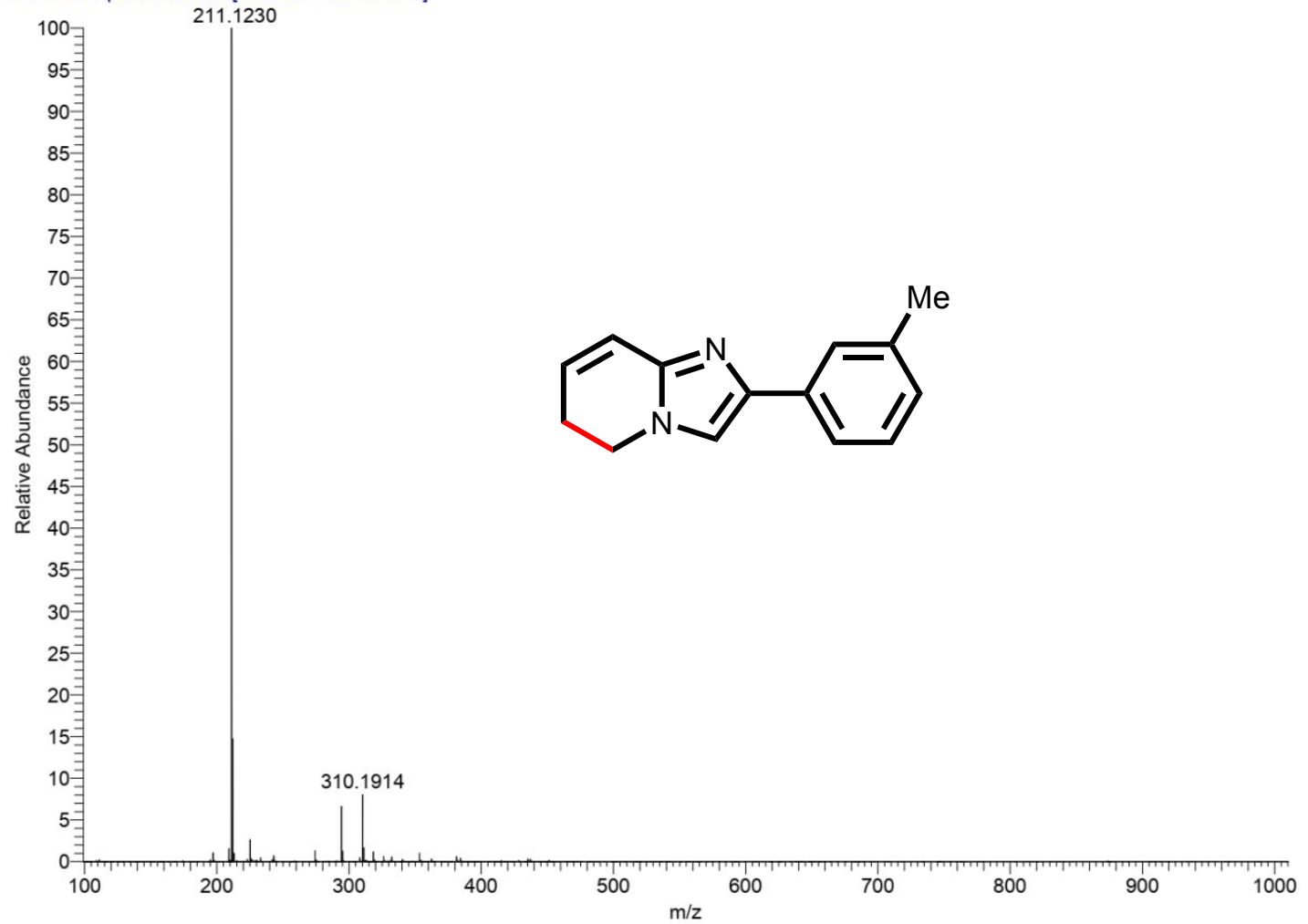


${ }^{1}$ HNMR

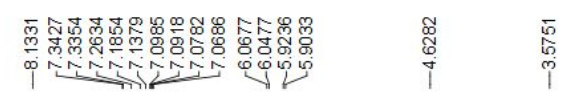

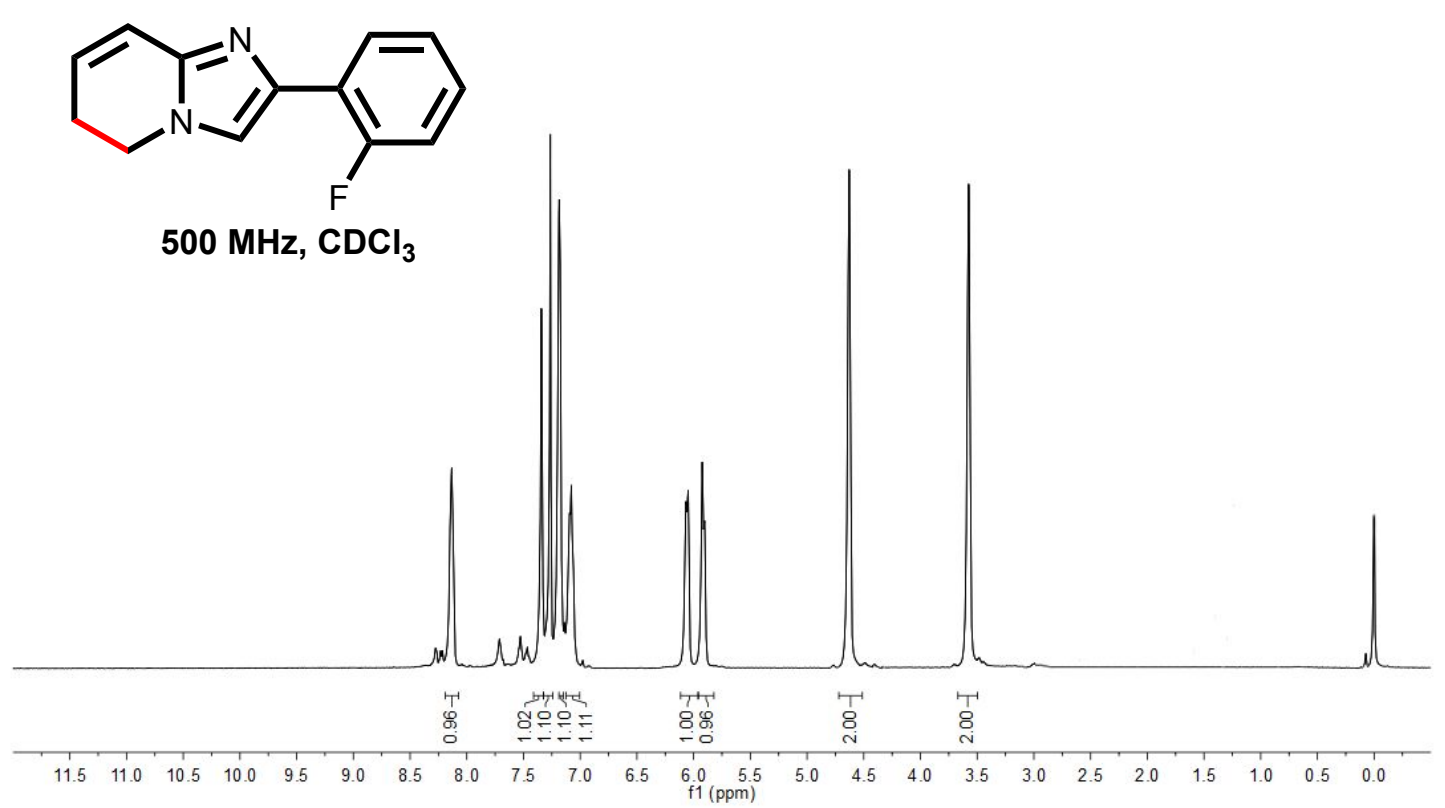

${ }^{13}$ CNMR

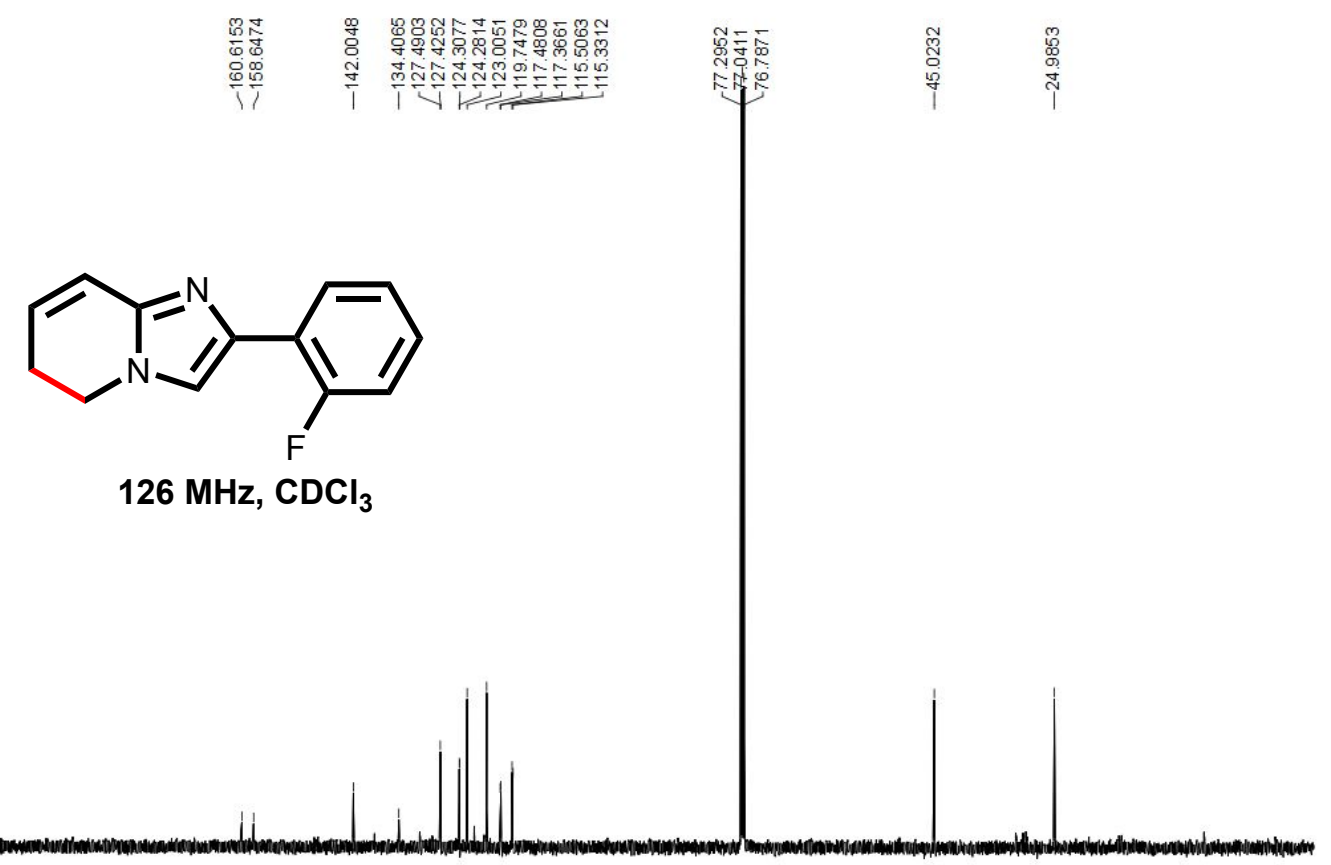

$\begin{array}{llllllllllllllllllllllllllllllll}1 & 200 & 190 & 180 & 170 & 160 & 150 & 140 & 130 & 120 & 110 & 100 & 10 & 10 & 70 & 60 & 50 & 40 & 30 & 20 & 10 & 0 & -10\end{array}$ 

WJW-18\#19-22 RT: $0.08-0.10$ AV: 4 SB: 195 0.84-1.18, $1.28-1.80$ NL: $1.59 E 10$
T: FTMS + p ESI Full ms [100.0000-1000.0000]

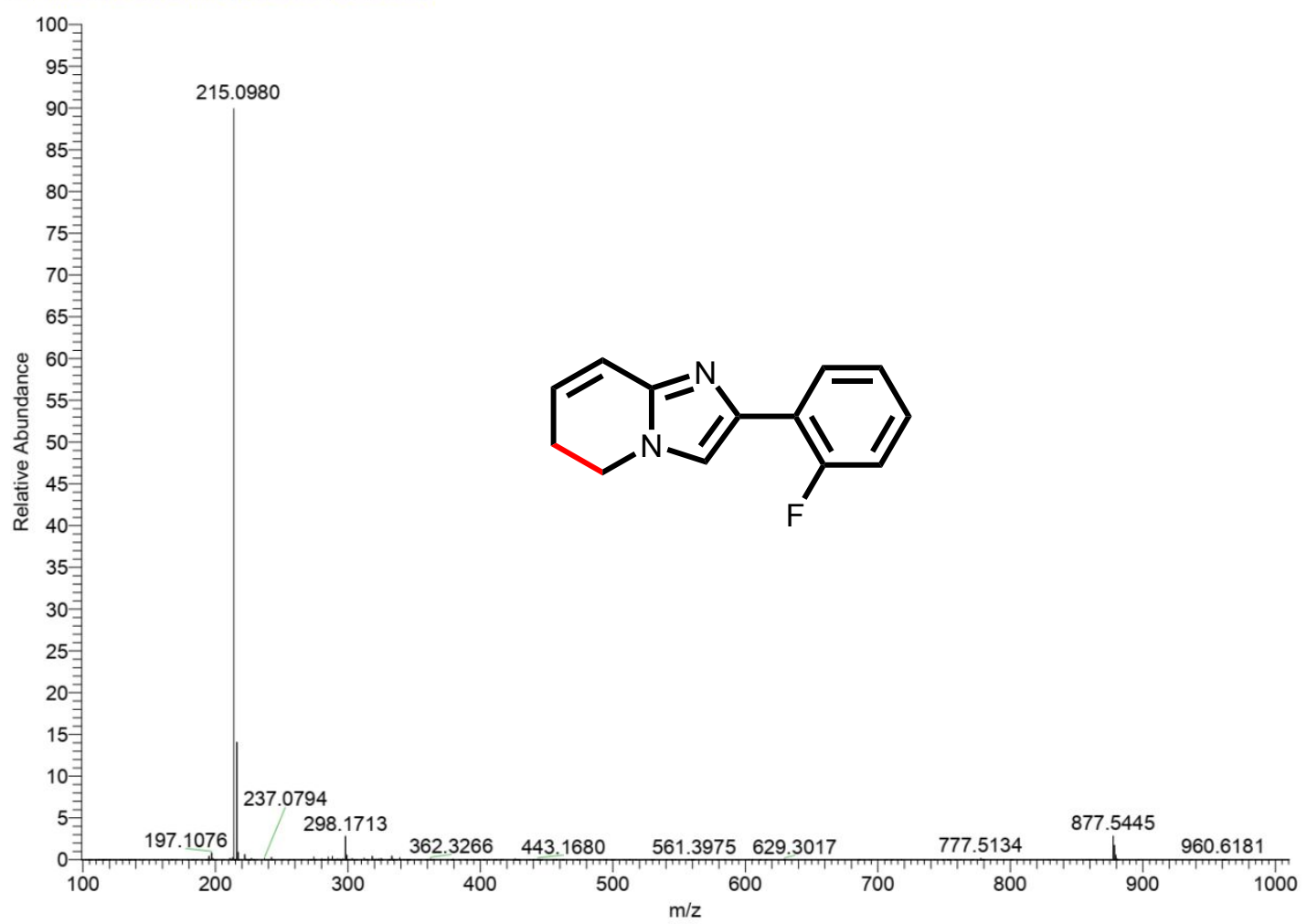


${ }^{1}$ HNMR

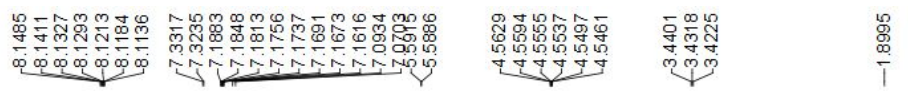

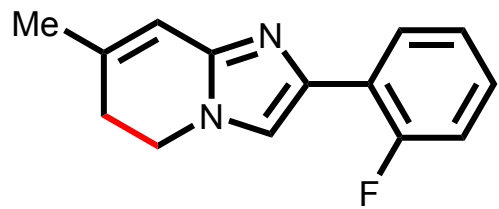

$500 \mathrm{MHz}, \mathrm{CDCl}_{3}$

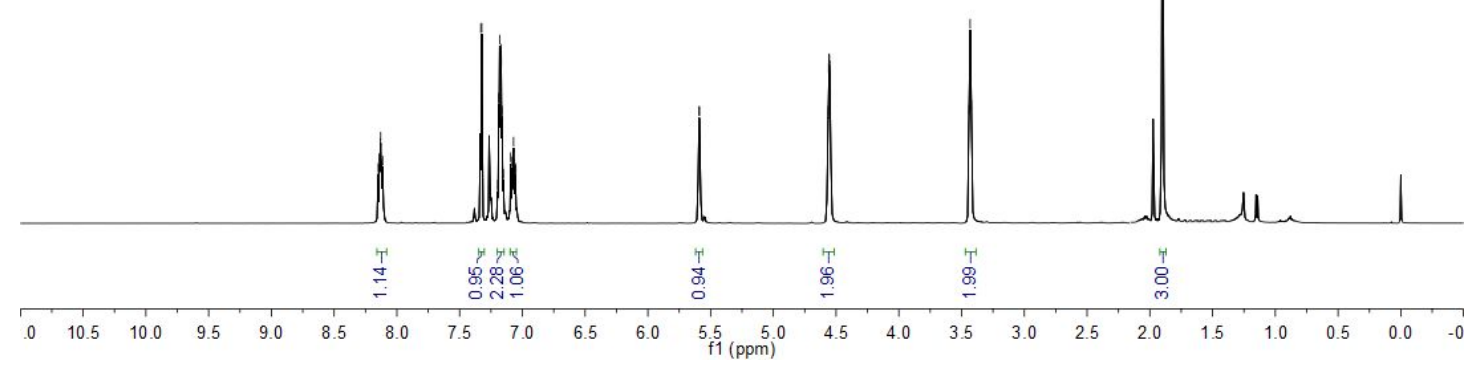

${ }^{13}$ CNMR

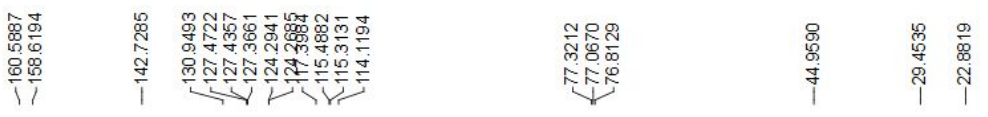<smiles>CC1=Cc2nc(-c3ccccc3F)cn2CC1</smiles>

$126 \mathrm{MHz}, \mathrm{CDCl}_{3}$

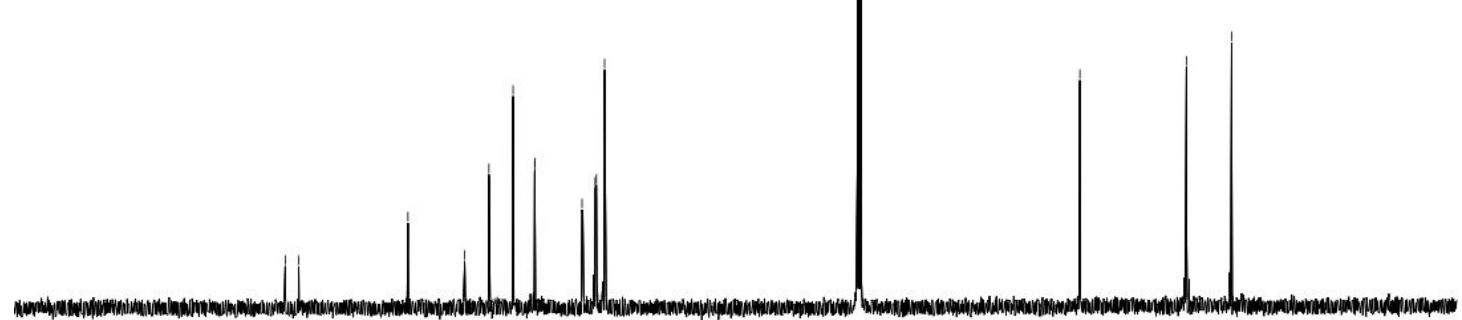

$\begin{array}{llllllllllllllllllllllllll}1 & 190 & 180 & 170 & 160 & 150 & 140 & 130 & 120 & 110 & 100 & 90 & 80 & 70 & 60 & 50 & 40 & 30 & 20 & 10 & 0 & -1\end{array}$ 
WJW-9\#14-25 RT: 0.06-0.10 AV: 11 SB: 75 0.80-1.13 NL: 8.57E9

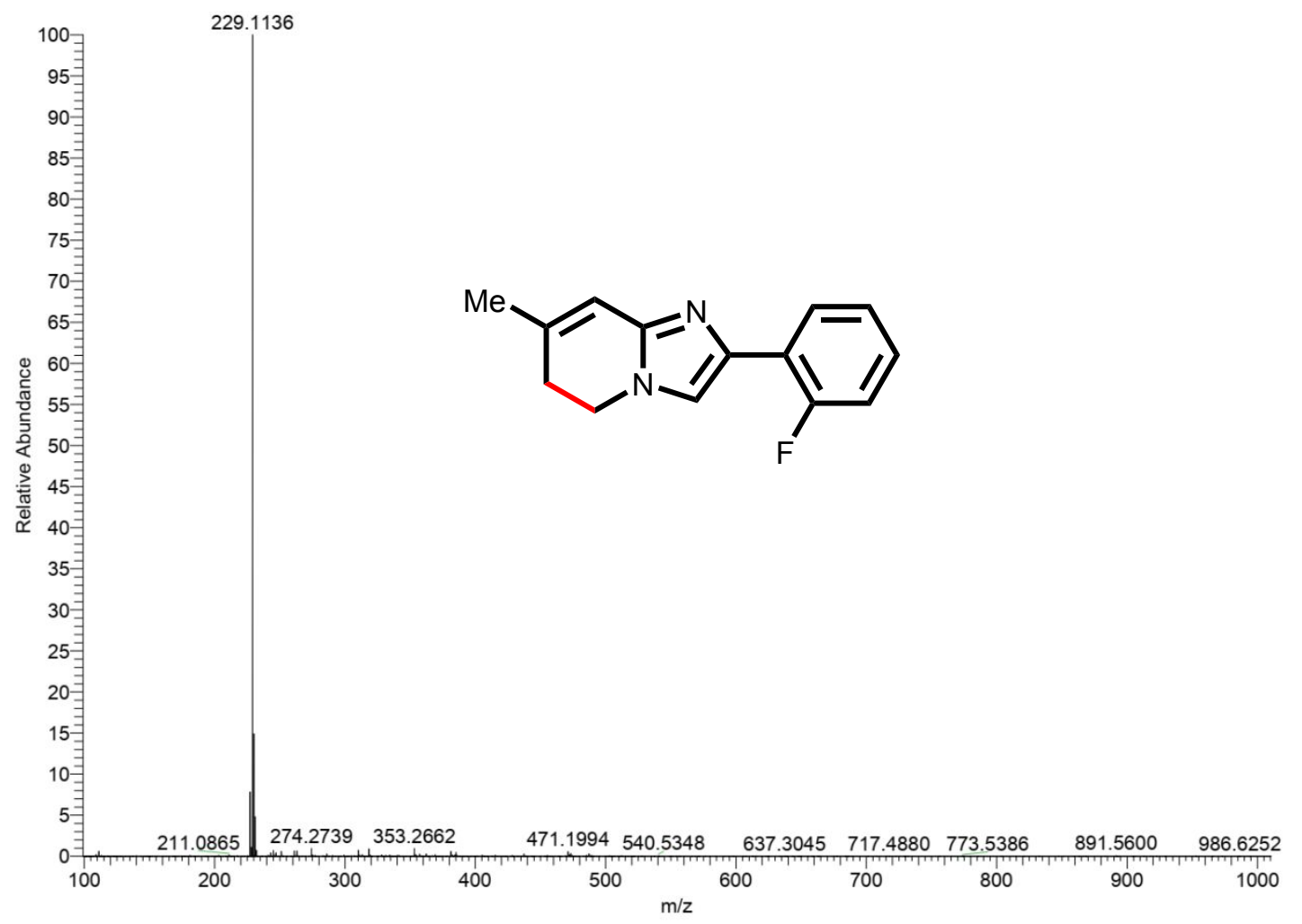


${ }^{1}$ HNMR

以ू.

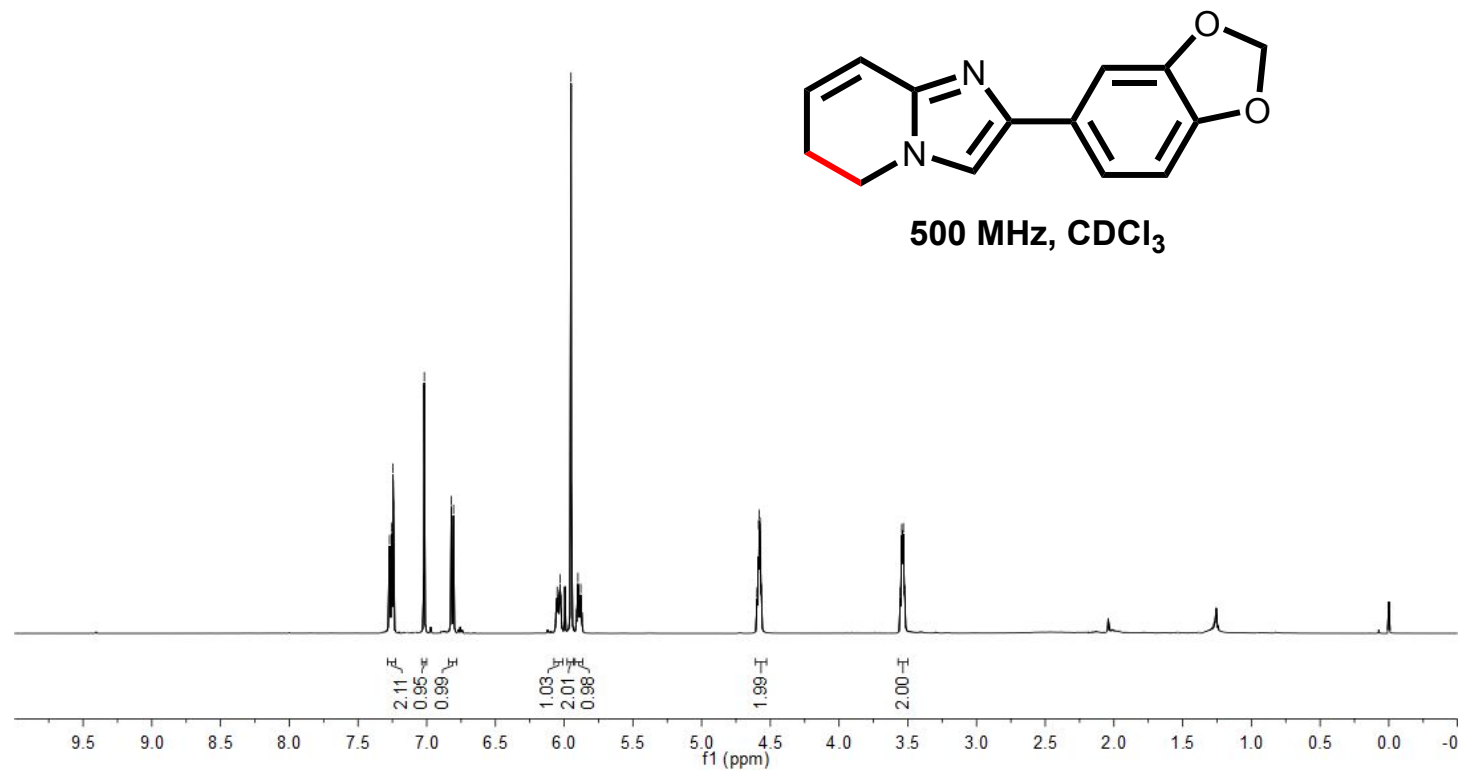

${ }^{13}$ CNMR

\begin{tabular}{|c|c|c|c|}
\hline 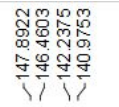 & 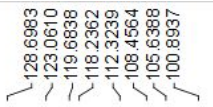 & 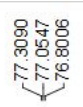 & \\
\hline
\end{tabular}

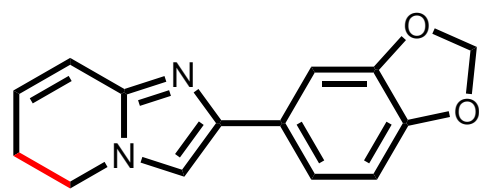

$126 \mathrm{MHz}^{\mathrm{CDCl}_{3}}$

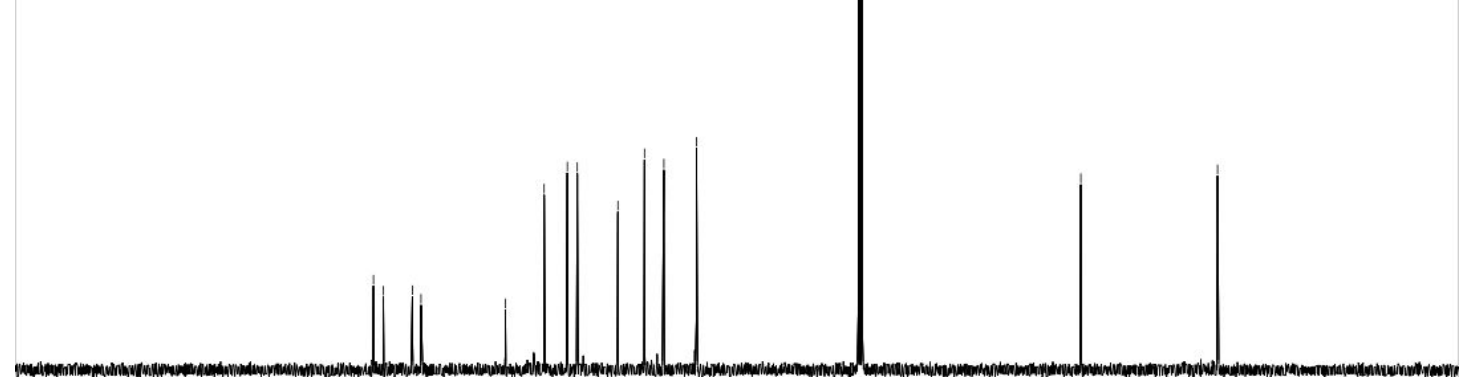

$\begin{array}{lllllllllll}190 & 180 & 170 & 160 & 150 & 140 & 130 & 120 & 110 & 100 & 9 \\ & & & & & & & & & & \end{array}$ 


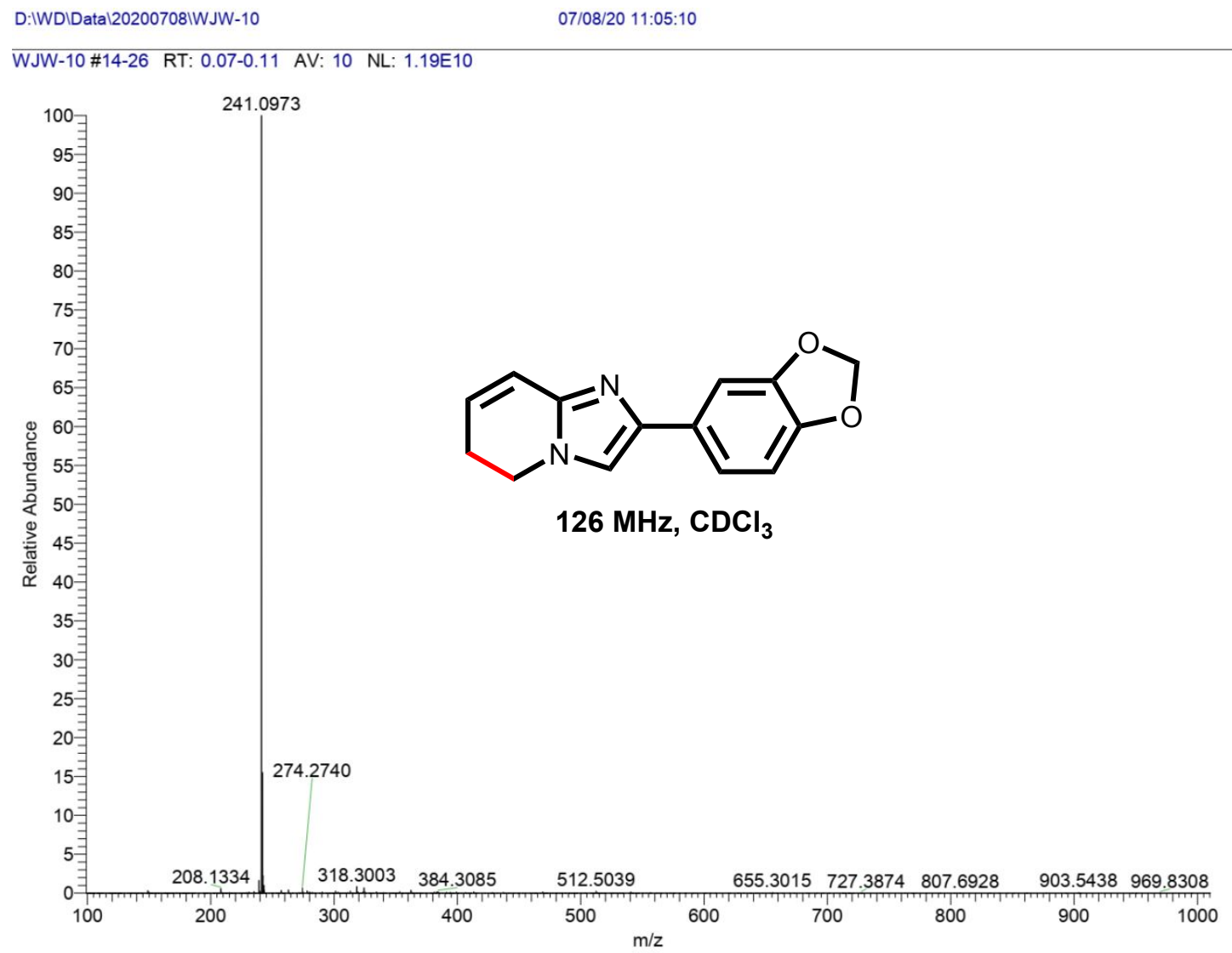


${ }^{1}$ HNMR

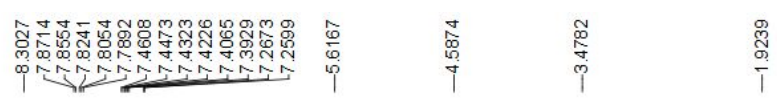<smiles>CC1=Cc2nc(-c3ccc4ccccc4c3)cn2CC1</smiles>

$500 \mathrm{MHz}^{\mathrm{CDCl}_{3}}$

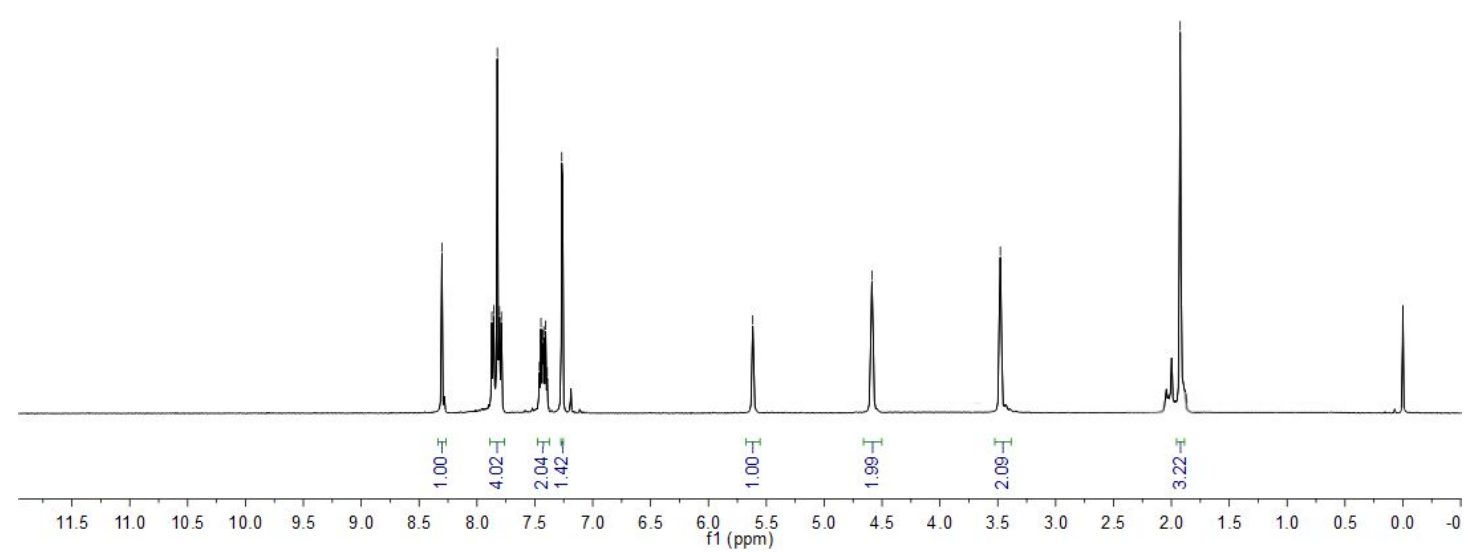

${ }^{13}$ CNMR

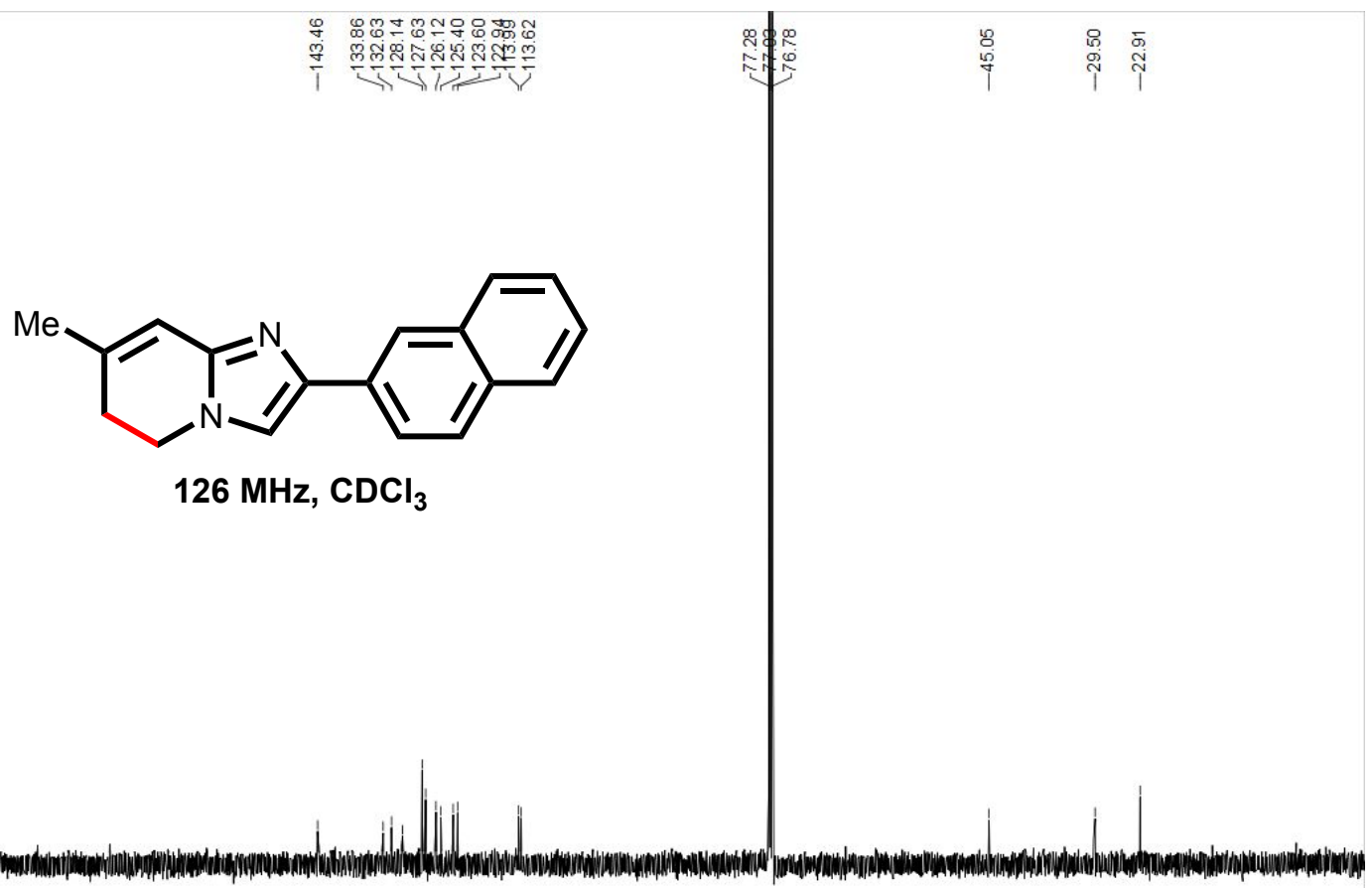

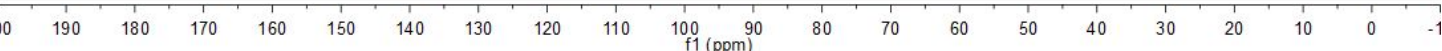


WJW-10\#16-25 RT: 0.07-0.11 AV: 10 SB: 127 1.09-1.65 NL: $1.72 E 7$

T: FTMS + p ESI Full ms [100.0000-1000.0000]

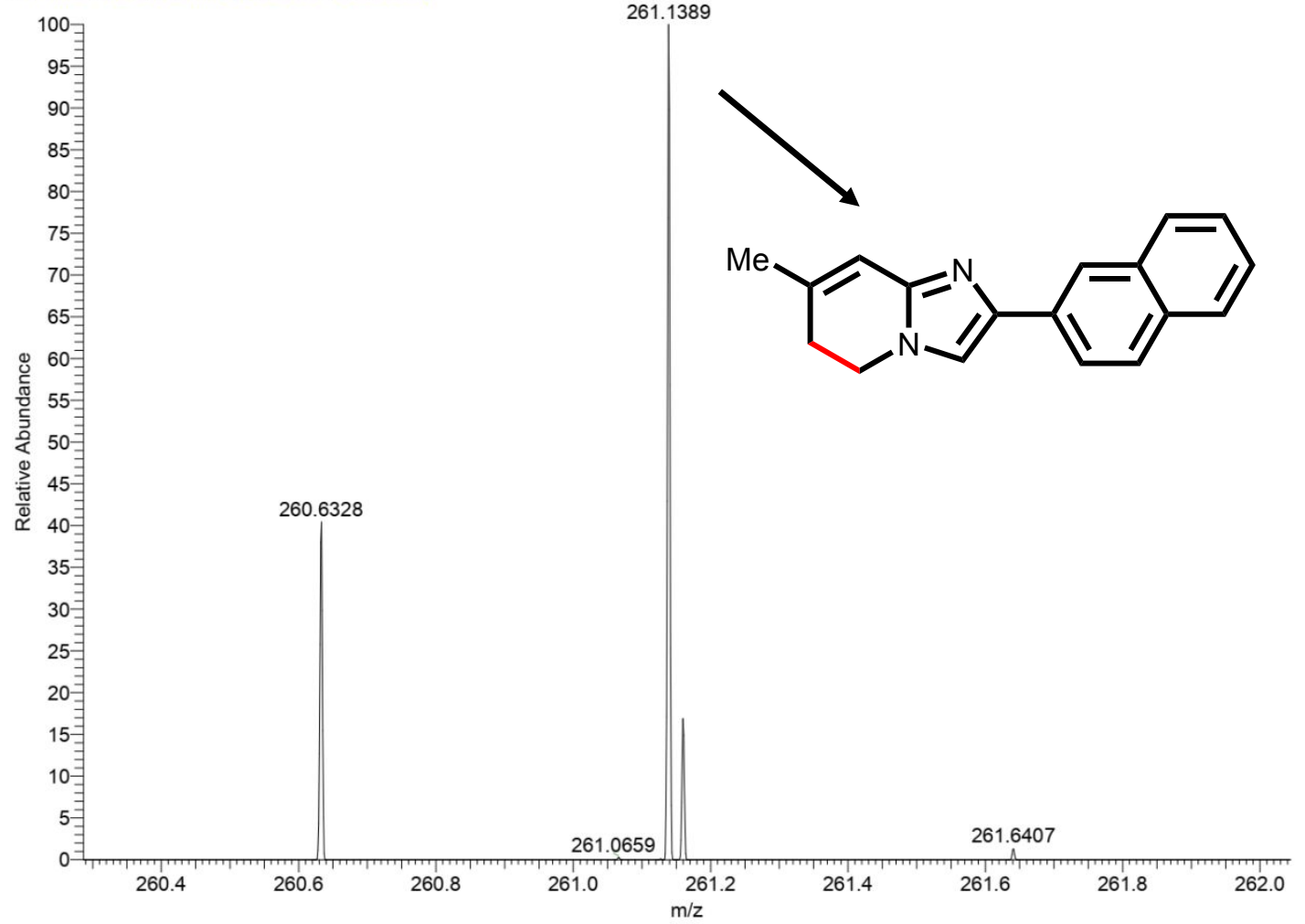




\section{${ }^{1}$ HNMR}

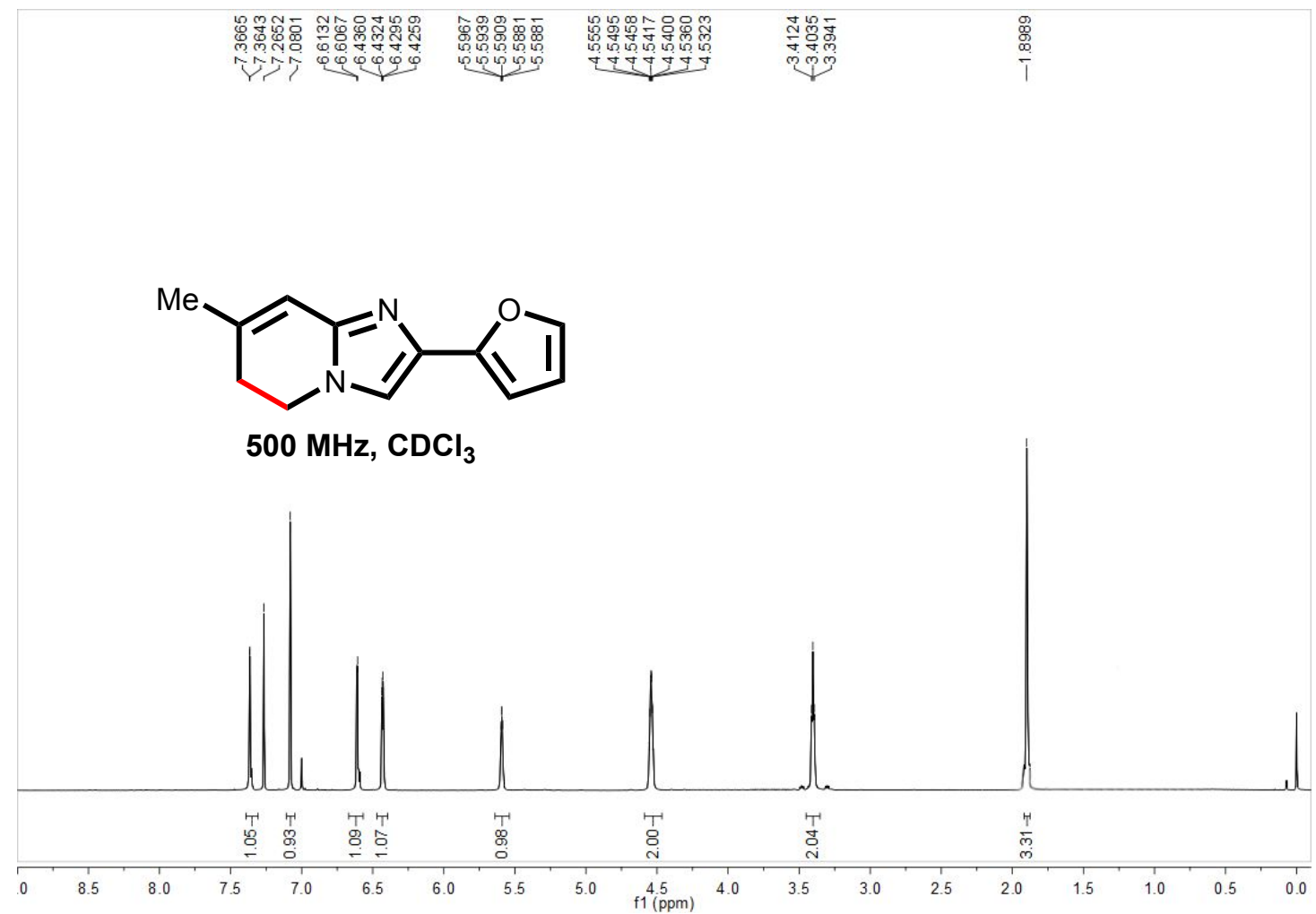

${ }^{13}$ CNMR

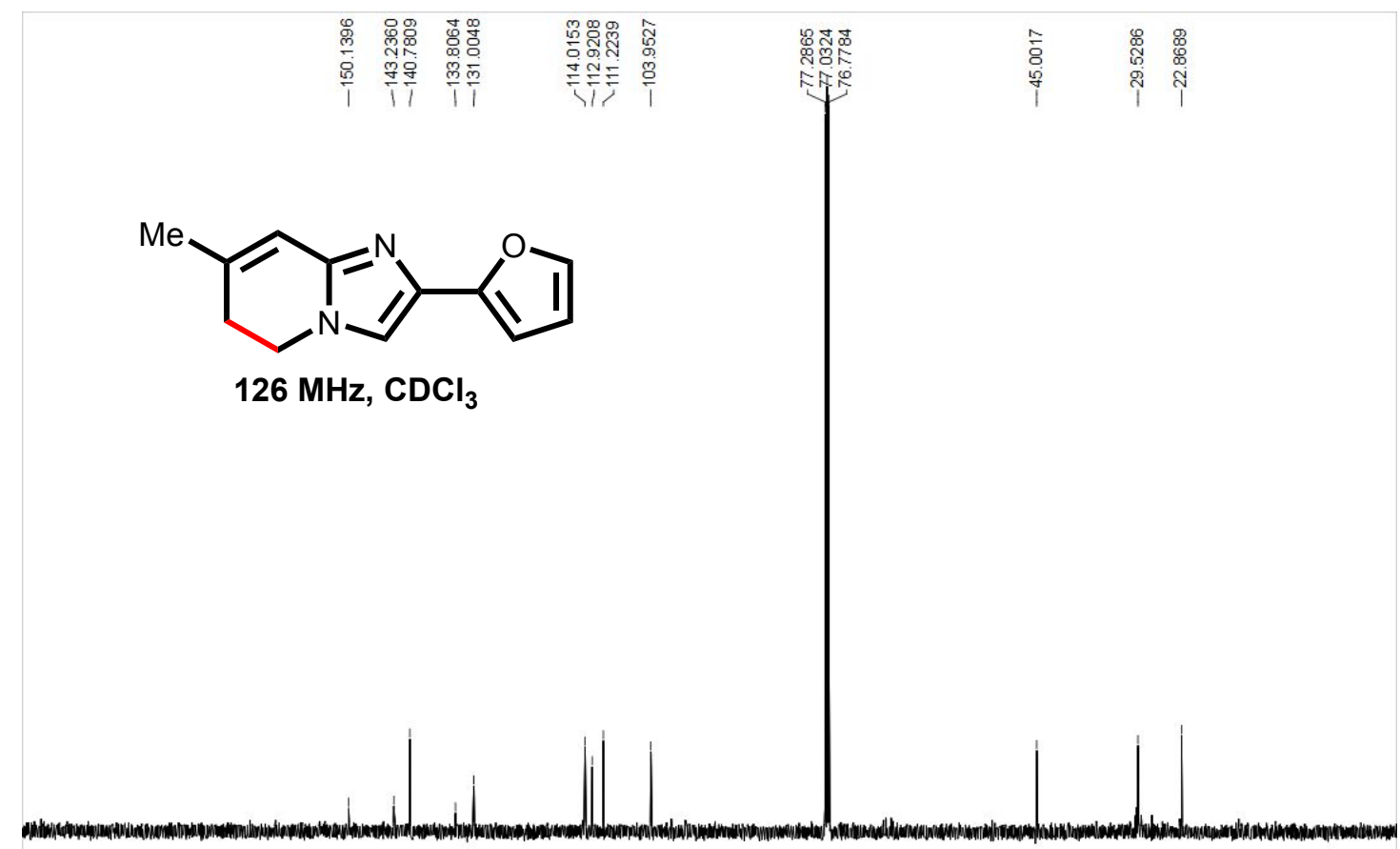

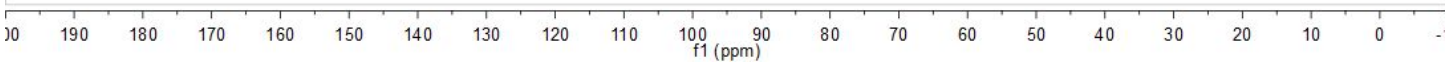


WJW-31 \#16-27 RT: 0.07-0.12 AV: 12 NL: 1.93E8

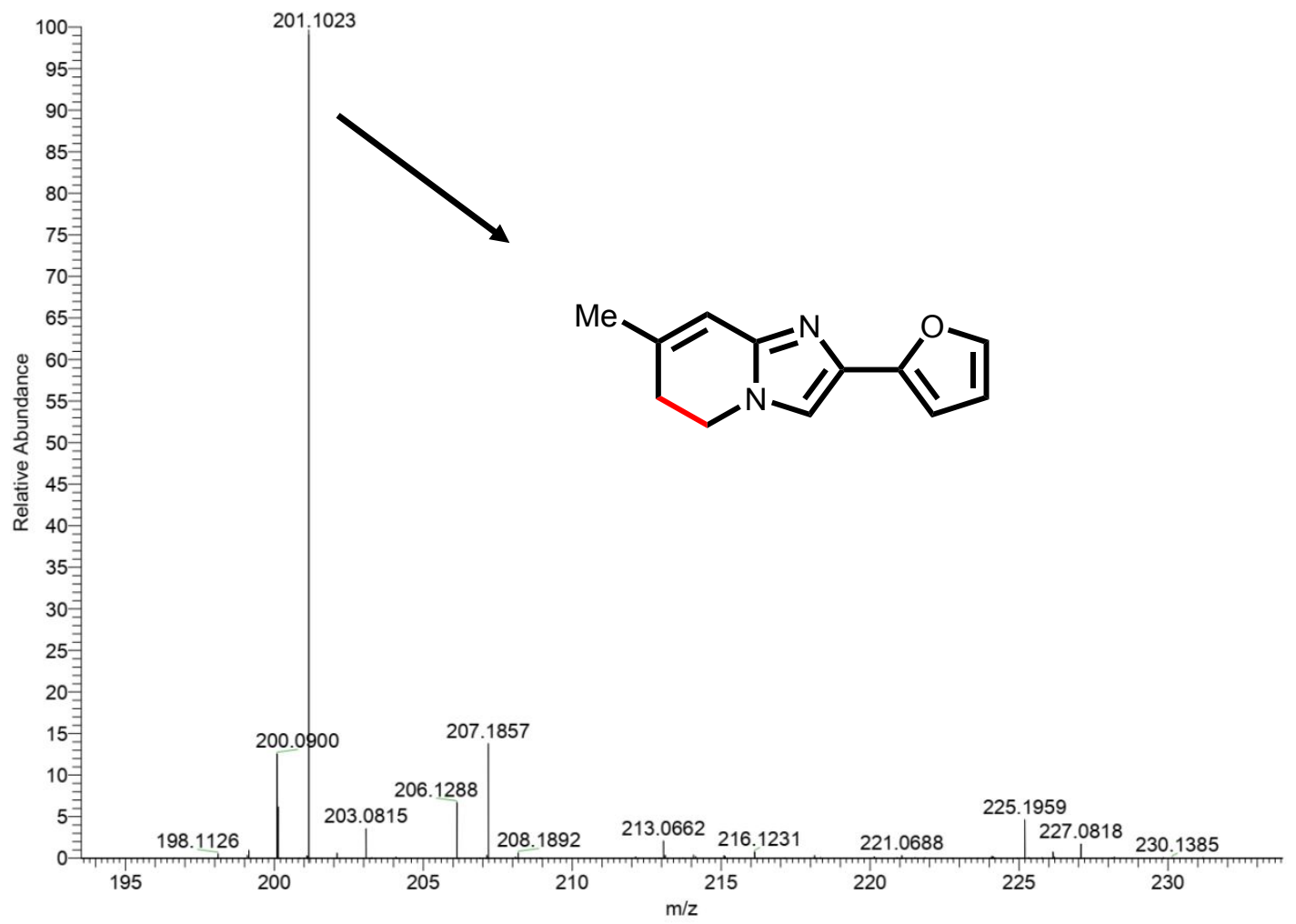


${ }^{1}$ HNMR

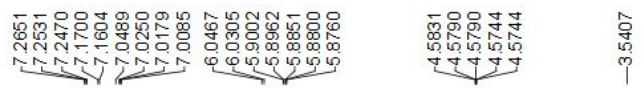<smiles>C1=Cc2nc(-c3cccs3)cn2CC1</smiles>

$500 \mathrm{MHz}, \mathrm{CDCl}_{3}$

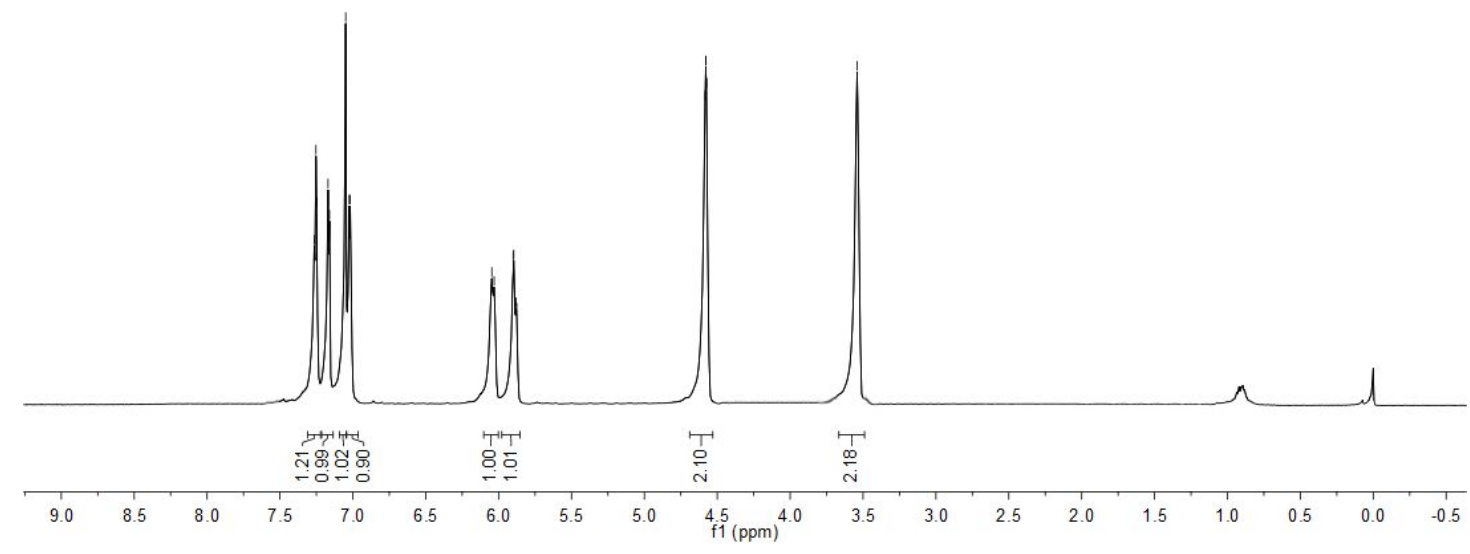

${ }^{13}$ CNMR

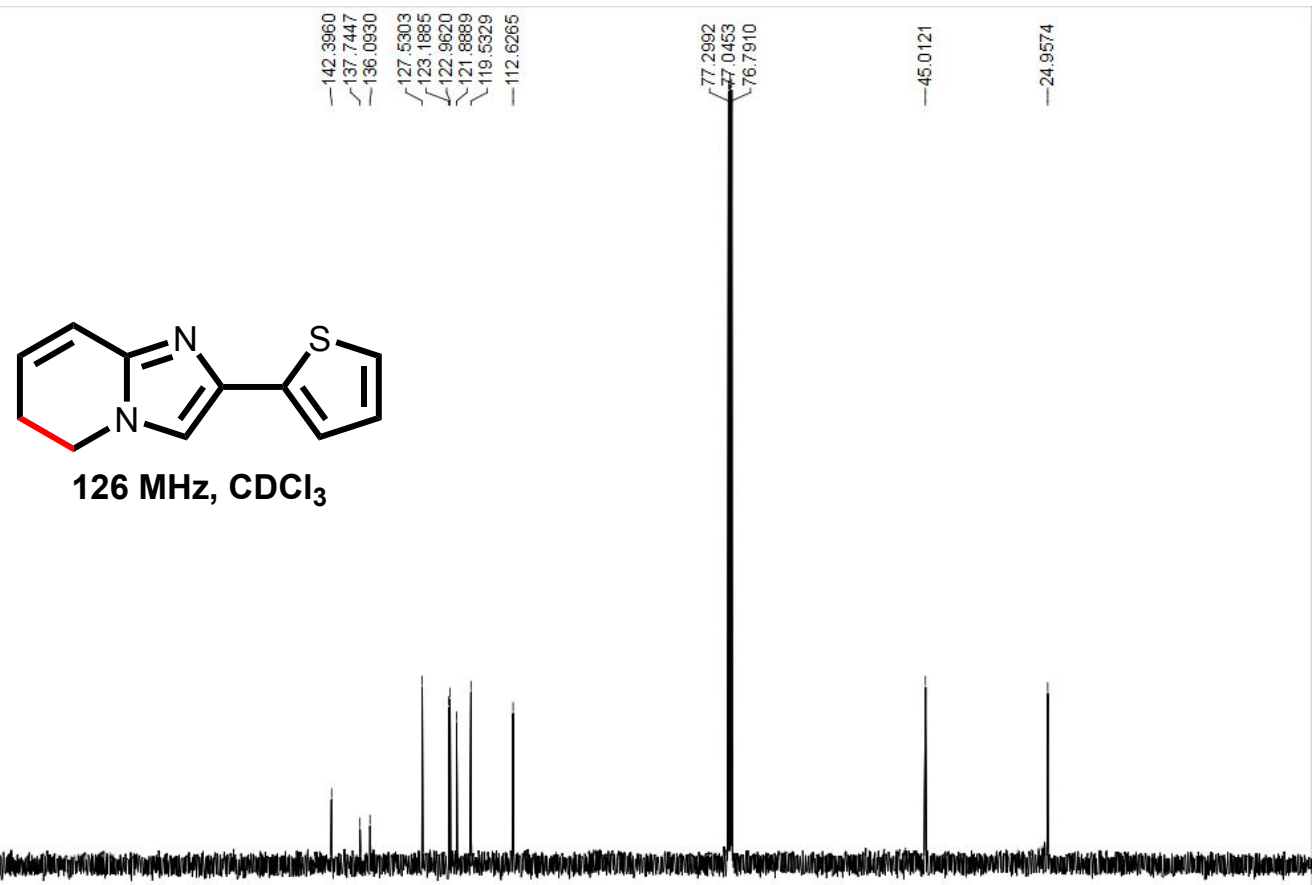

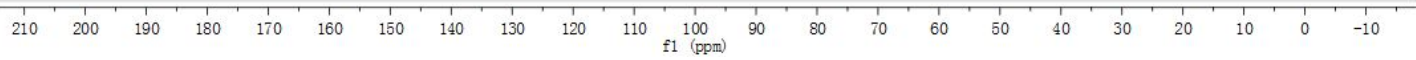


WJW-32 \#16-28 RT: 0.07-0.12 AV: 12 NL: 1.93E8

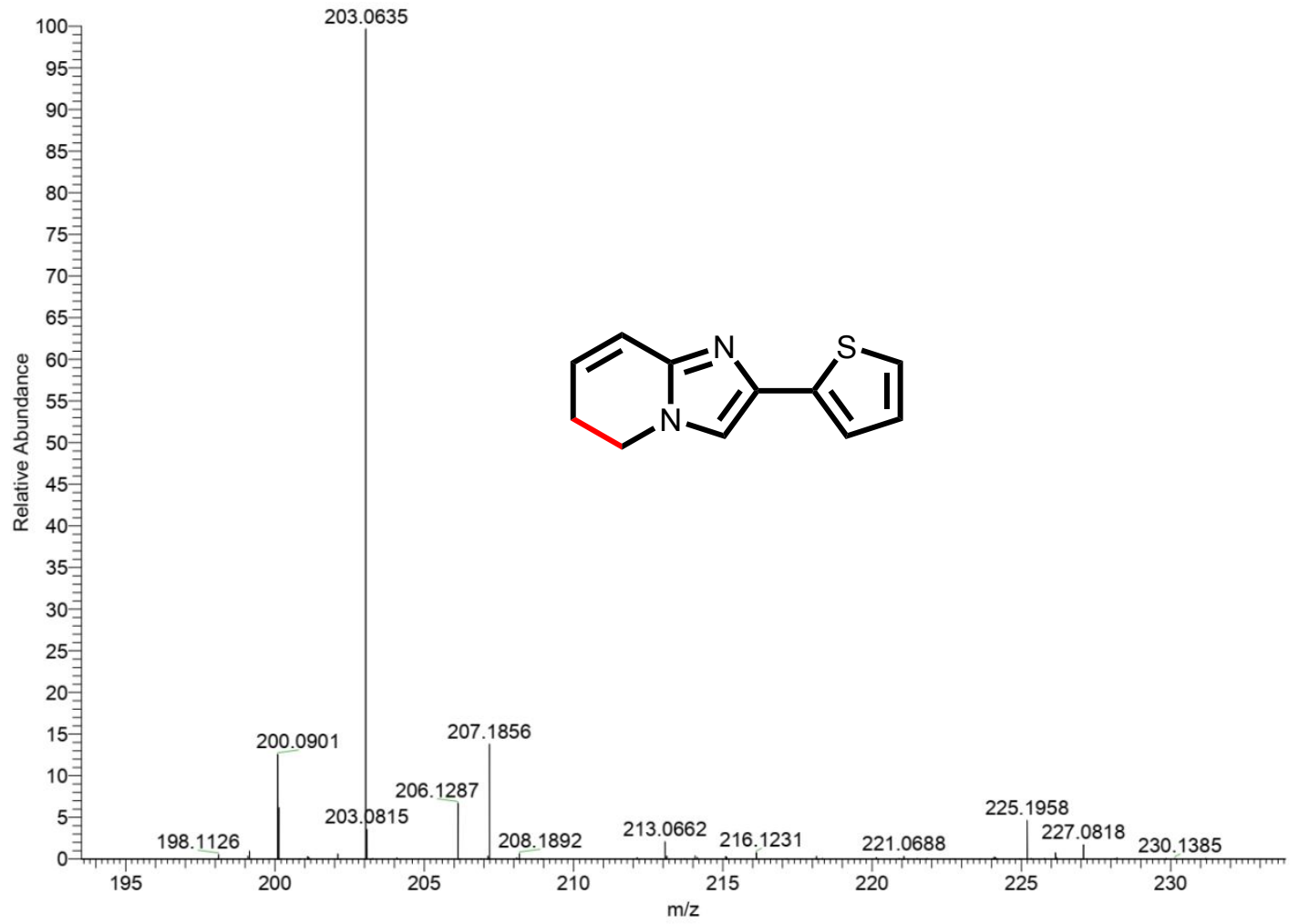

